EDITORIAL BOARD

ANTONIO CARCATERRA

ERIC A. CARLEN

FRANCESCO DELL'ISOLA

RAFFAELE ESPOSITO

ALBERT FANNJIANG

Gilles A. FranCFORT

Pierangelo MARCATI

JEAN-JACQUES MARIGO

PETER A. MARKOWICH

MARTIN OSTOJA-STARZEWSKI

PIERRE SEPPECHER

DAVID J. STEIGMANN

PAUl STEINMANN

PierRe M. SuQueT

MANAGING EDITORS

MICOL AMAR

CORRADO LATTANZIO

ANGELA MADEO

MARTIN OSTOJA-STARZEWSKI

ADVISORY BOARD

ADNAN AKAY

Holm AltenBaCH

MICOL AMAR

HARM ASKES

TEODOR ATANACKOVIĆ

VICTOR BERDICHEVSKY

GUY BOUCHITTÉ

ANDREA BRAIDES

ROBERTO CAMASSA

MAURO CARFORE

ERIC DARVE

FELIX DARVE

ANNA DE MASI

GianPiEtro DEL Piero

EMMANUELE Di BENEDETTO

BERNOLD FIEDLER

IRENE M. GAMBA

DAVID Y. GAO

SERGEY GAVRILYUK

TIMOTHY J. HEALEY

DOMINIQUE JEULIN

ROGER E. KHAYAT

CORRADO LATTANZIO

ROBERT P. LIPTON

ANGELO LUONGO

ANGELA MADEO

JUAN J. MANFREDI

CARLO MARCHIORO

GÉRARD A. MAUGIN

ROBERTO NATALINI PATRIZIO NEFF

ANDREY PIATNITSKI

ERRICO PRESUTTI

MARIO PULVIRENTI

LUCIO RUSSO

Miguel A. F. SANJUAN

PATRICK SElVADURAI

ALEXANDER P. SEYRANIAN

MIROSLAV ŠILHAVÝ

GUIDO SWEERS

ANTOINETTE TORDESILLAS

LEV TRUSKINOVSKY

JUAN J. L. VELÁZQUEZ VINCENZO VESPRI ANGELO VULPIANI msp.org/memocs

Università di Roma "La Sapienza", Italia

Rutgers University, USA

(CO-CHAIR) Università di Roma "La Sapienza", Italia

(TREASURER) Università dell'Aquila, Italia

University of California at Davis, USA

(CO-CHAIR) Université Paris-Nord, France

Università dell'Aquila, Italy

École Polytechnique, France

DAMTP Cambridge, UK, and University of Vienna, Austria

(CHAIR MANAGING EDITOR) Univ. of Illinois at Urbana-Champaign, USA

Université du Sud Toulon-Var, France

University of California at Berkeley, USA

Universität Erlangen-Nürnberg, Germany

LMA CNRS Marseille, France

Università di Roma "La Sapienza", Italia

Università dell'Aquila, Italy

Université de Lyon-INSA (Institut National des Sciences Appliquées), France

(CHAIR MANAGING EDITOR) Univ. of Illinois at Urbana-Champaign, USA

Carnegie Mellon University, USA, and Bilkent University, Turkey

Otto-von-Guericke-Universität Magdeburg, Germany

Università di Roma "La Sapienza", Italia

University of Sheffield, UK

University of Novi Sad, Serbia

Wayne State University, USA

Université du Sud Toulon-Var, France

Università di Roma Tor Vergata, Italia

University of North Carolina at Chapel Hill, USA

Università di Pavia, Italia

Stanford University, USA

Institut Polytechnique de Grenoble, France

Università dell'Aquila, Italia

Università di Ferrara and International Research Center MEMOCS, Italia

Vanderbilt University, USA

Freie Universität Berlin, Germany

University of Texas at Austin, USA

Federation University and Australian National University, Australia

Université Aix-Marseille, France

Cornell University, USA

École des Mines, France

University of Western Ontario, Canada

Università dell' Aquila, Italy

Louisiana State University, USA

Università dell'Aquila, Italia

Université de Lyon-INSA (Institut National des Sciences Appliquées), France University of Pittsburgh, USA

Università di Roma "La Sapienza”, Italia

Université Paris VI, France

Istituto per le Applicazioni del Calcolo "M. Picone", Italy

Universität Duisburg-Essen, Germany

Narvik University College, Norway, Russia

Università di Roma Tor Vergata, Italy

Università di Roma "La Sapienza”, Italia

Università di Roma “Tor Vergata”, Italia

Universidad Rey Juan Carlos, Madrid, Spain

McGill University, Canada

Moscow State Lomonosov University, Russia

Academy of Sciences of the Czech Republic

Universität zu Köln, Germany

University of Melbourne, Australia

École Polytechnique, France

Bonn University, Germany

Università di Firenze, Italia

Università di Roma La Sapienza, Italia

MEMOCS (ISSN 2325-3444 electronic, 2326-7186 printed) is a journal of the International Research Center for the Mathematics and Mechanics of Complex Systems at the Università dell'Aquila, Italy.

Cover image: "Tangle” by $\odot$ John Horigan; produced using the Context Free program (contextfreeart.org).

PUBLISHED BY

7 mathematical sciences publishers

nonprofit scientific publishing

http://msp.org/

(C) 2016 Mathematical Sciences Publishers 


\title{
CONSTRAINT REACTION AND THE PEACH-KOEHLER FORCE FOR DISLOCATION NETWORKS
}

\author{
RicCARdo ScAla AND NicOlas VAN GoETHEM
}

In the presence of dislocations, the elastic deformation tensor $F$ is not a gradient but satisfies the condition Curl $F=\Lambda_{\mathscr{L}}^{\mathrm{T}}$ (with the dislocation density $\Lambda_{\mathscr{L}}$ a tensor-valued measure concentrated in the dislocation $\mathscr{L}$ ). Then $F \in L^{p}$ with $1 \leq p<2$. This peculiarity is at the origin of the mathematical difficulties encountered by dislocations at the mesoscopic scale, which are here modeled by integral 1-currents free to form complex geometries in the bulk. In this paper, we first consider an energy-minimization problem among the couples $(F, \mathscr{L})$ of strains and dislocations, and then we exhibit a constraint reaction field arising at minimality due to the satisfaction of the condition on the deformation curl, hence providing explicit expressions of the Piola-Kirchhoff stress and PeachKoehler force. Moreover, it is shown that the Peach-Koehler force is balanced by a defect-induced configurational force, a sort of line tension. The functional spaces needed to mathematically represent dislocations and strains are also analyzed and described in a preliminary part of the paper.

\section{Introduction}

Dislocations in elastic bodies are at the origin of dissipative phenomena, and in particular, their motion is responsible for the plastic behavior of single crystals. A dislocation loop $L$ is a closed curve in $\bar{\Omega}$. Outside the dislocation, i.e., in $\bar{\Omega} \backslash L$, the body is considered perfectly elastic. This scale of matter description is called the mesoscopic or the continuum scale. Nonetheless, it is not easy to understand the physical nature of a mesoscopic dislocation. In fact, it is not a material line since it can be equivalently generated by an excess or a lack of lattice atoms. Moreover, contrarily to fracture, it cannot even be defined as a mere singularity in the reference configuration where deformation fields would be unbounded. In fact, a dislocation must be viewed as a singularity of the deformation field whose support lies in the current configuration (see, e.g., [Acharya 2003; Scala and Van Goethem 2016]). Therefore, dislocation location and field singularity are bound notions.

\section{Communicated by Francesco dell'Isola.}

MSC2010: 49Q15, 74B20, 74G65.

Keywords: dislocations, finite elasticity, variational problem, Peach-Koehler force, constraint reaction, integer-multiplicity currents. 


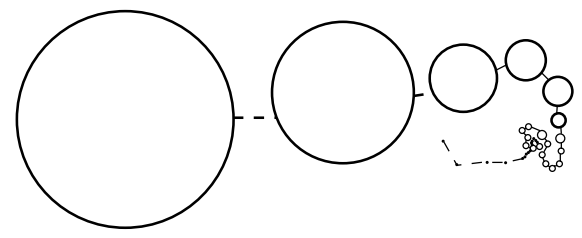

Figure 1. Example of a continuum dislocation cluster.

Specifically, the support of the curl of the deformation field (which in the presence of dislocations is not a gradient) is identified with the dislocation density field. This definition is at the basis of the present work since a constraint reaction will be generated by the satisfaction of the latter relation between model variables.

1.1. Mathematical and physical properties of dislocations. The intrinsic mathematical difficulties generated by dislocations are fundamentally different from those encountered in the mathematical modeling of fracture mechanics. In particular, the displacement is not an appropriate model variable as opposed to most of solid mechanics problems. Furthermore, the stress and strain fields are not squareintegrable and so the less-tractable $L^{p}$ spaces with $1 \leq p<2$ must be considered, and bounds on the model fields are given in terms of the curl and the divergence (in place of the full gradient) in measure spaces (instead of Sobolev spaces). Moreover, we believe that in order to model single crystals with dislocations, where complex geometries such as dislocation networks (see Figure 1) are observed [Zaiser 2004], one can hardly rely on the assumption of a periodic array of straight dislocations. Therefore, one is forced to build specific mathematical tools step by step, which should provide

- an appropriate functional framework and

- a geometric description of the lines.

To achieve the latter, the mathematical formalism of currents as briefly described in Section 1.2 has been proposed. In this framework, a cluster as depicted in Figure 1 is modeled as a continuum dislocation [Scala and Van Goethem 2016]. The formalism of currents to study and model dislocation clusters has been introduced in the pioneering works [Hochrainer and Zaiser 2005; Hochrainer 2013] and then adopted in more recent contributions to the theory of continuum dislocations as in [Conti et al. 2015a; 2015b]. The notion of integral current with coefficient in a group, also adopted in the companion paper [Scala and Van Goethem 2016; 2015], is the main tool to treat dislocation networks. This is due, principally, to the ability of dislocation lines to annihilate, sum, and form complex structures with specific rules for summing the Burgers vectors, which belong to a specific group. Moreover, the formalism of currents in general has proven to be useful in treating variational 
problems in the theory of continuum dislocations [Scala and Van Goethem 2016; 2015]. Restricting ourselves to a quasistatic regime, we assume that the optimal networks result from minimization laws (note that such minimization states are reached very fast in actual crystals such as pure copper, where resistance to dislocation motion is negligible [Berdichevsky 2006]).

Therefore, the first purpose of this paper is to establish the functional setting appropriate to describe mesoscopic dislocations. The main features are that, when Sobolev spaces $W^{1, p}$ are considered, exponent $p$ is in the "bad range" $1 \leq p<\frac{3}{2}$ and that the second grade variable is the curl instead of the gradient and the curl must be a concentrated Radon measure. Minimization problems in this range are considered in [Scala and Van Goethem 2016], where, aware of [Müller and Palombaro 2008], the main tools used are integral currents and Cartesian maps.

We shall provide elements for an analysis of the space of $L^{p}$-tensors whose curl is bounded in a measure space and in particular bring to light and study the homeomorphism between this space and the space of solenoidal Radon measures, which in the model application will be the space of dislocation densities. The second purpose is to compute the first variation of the energy with respect to the strain and the dislocation-associated density. These will allow us to determine a configurational force, capable of driving the dislocations outside equilibrium, which, as far as the deformation part of the energy is concerned, is the well-known Peach-Koehler force.

\subsection{A quick survey on currents and dislocations at the continuum scale. In} [Scala and Van Goethem 2016], we proposed a mathematical model for a countable family of dislocations in an elastic body $\Omega$, here considered the current (as opposed to "reference") configuration. Motivated by physical reasons [Hirth and Lothe 1982; Müller et al. 2004; Zaiser 2004; Zubov 1997], we consider finite elasticity near the line with a less-than-quadratic strain energy while linear elasticity is a valid assumption away from the dislocations. Since the dislocation loop is the singularity set for stress and strain, the deformation gradient field $F$ is incompatible, meaning ${ }^{1}$

$$
-\operatorname{Curl} F=\Lambda^{\mathrm{T}} \neq 0 \quad \text { in } \Omega,
$$

with $F$ the (inverse) deformation tensor and where the dislocation density $\Lambda$ is a Radon measure in $\mathcal{M}\left(\bar{\Omega}, \mathbb{M}^{3}\right)$ concentrated on the dislocation set $L$. Here, $L$ is a dislocation network in the current (i.e., deformed) configuration. Clearly if $\Lambda=0$, then $F$ is a gradient and there are no dislocations in the bulk. Moreover, conservation properties for dislocations imply that their density is solenoidal:

$$
\operatorname{Div} \Lambda^{\mathrm{T}}=0 .
$$

\footnotetext{
${ }^{1}$ Componentwise, $(\operatorname{Curl} F)_{i j}=\epsilon_{j k l} \partial_{k} F_{i l}$ and $\Lambda_{i j}=\tau_{i} b_{j} \delta \mathscr{L}$.
} 
The explicit formula for $\Lambda$ shows a linear dependence on the line orientation $\tau$ and on the Burgers vector $b$ (i.e., $\Lambda:=\tau \otimes b \delta_{L}$ ), where for crystallographic reasons the value of the Burgers vector is constrained to belong to a countable lattice in $\mathbb{R}^{3}$.

In the proposed formalism, currents (for which the main reference is [Federer 1969]) are used to describe dislocations at the mesoscopic scale. Specifically, dislocations are described by integer-multiplicity 1-currents, which are mathematical objects generalizing the concept of curves and are assumed closed to account for the property (1-2), implying that every dislocation is a loop or ends at the crystal boundary. A brief survey of the mathematical formalism can be found in Section 3.1 while for details we refer to [Scala and Van Goethem 2016]. For a so-called dislocation current $\mathscr{L}$, we will denote the associated density by $\Lambda=\Lambda_{\mathscr{L}}$. Whatever the model may be, in this paper, we are merely concerned with variations at optimality; thus, modeling and existence issues are not discussed.

The starting point of the present work is the minimum problem

$$
\min _{(F, \mathscr{L}) \in \mathscr{A}} \mathscr{W}\left(F, \Lambda_{\mathscr{L}}\right)
$$

where the energy

$$
\mathcal{W}\left(F, \Lambda_{\mathscr{L}}\right)=\mathcal{W}_{\mathrm{e}}(F)+\mathcal{W}_{\text {dislo }}\left(\Lambda_{\mathscr{L}}\right)
$$

satisfies some appropriate convexity and coerciveness conditions while $\mathscr{A}$ is the space of admissible couples of deformation and dislocation currents. Among the properties of admissibility, we require that $F$ and $\mathscr{L}$ be related by condition (1-1) and that $F$ be the gradient of a Cartesian map away from $L$. Therefore, both $F$ and $\mathscr{L}$ are represented by particular types of integral currents.

In dislocation gauge theory, an energy like (1-4) was used in [Lazar and Anastassiadis 2008; Agiasofitou and Lazar 2010], where the decomposition in an elastic and a dislocation part is given. From a mathematical viewpoint, that is, with variational techniques in appropriate functional spaces, problem (1-3) has been discussed and was first solved in [Müller and Palombaro 2008] with a single fixed dislocation loop in the crystal bulk (thus implying a minimization in $F$ only) and later extended in [Scala and Van Goethem 2016] for an unfixed countable family of dislocation currents satisfying certain boundary conditions. Existence of minimizers is based on the assumption (classical in fracture mechanics) that the number of clusters is bounded.

1.3. Formal derivation of the Peach-Koehler force. From the standpoint of configurational force theory (as in [Gurtin 2000]) or as a result of invariance properties and Noether's theorem (as proposed by [Agiasofitou and Lazar 2010]), the Eshelby stress $\mathscr{E}=W \rrbracket-F^{\mathrm{T}} \mathbb{P}$ appears as a crucial quantity with $W$ the energy density and $\mathbb{P}$ the first Piola-Kirchhoff stress. Assuming that $\operatorname{Div} \mathbb{P}=0$, i.e., that static 
equilibrium holds, one immediately finds that

$$
\text { Peach-Koehler force }=\operatorname{Div} \mathscr{E}=\left\langle\Lambda_{\mathscr{L}}^{\mathrm{T}} \times \mathbb{P}\right\rangle,
$$

where the brackets emphasize that the vector product takes place in a certain function space as a duality product. Equation (1-5) is known as the Peach-Koehler force (see, e.g., [Hirth and Lothe 1982] for a straight dislocation); it is a force due to the equilibrium between the dislocation and the adjacent elastic medium. In particular, the functional choice is provided by the physics considered, that is, in the case of dislocations, whether one considers the macroscale (with Sobolev fields and no line singularity) or on the contrary the mesoscale, as in this work, where geometric measure theory and related functional spaces must be considered. Let us remark that the strong form $\operatorname{Div} \mathbb{P}=0$ is classically obtained by the Euler-Lagrange equation $\int_{\Omega} \mathbb{P} \cdot \nabla u d x=0$ for all test functions $u$, provided the integration by parts is valid. The point is that, at our scale of matter description, $\mathbb{P}$ turns out to belong to a Lebesgue space, and thus, $\mathbb{P} N$ is not defined at the boundary, precluding the use of the divergence theorem. For this reason, Div $\mathbb{P}=0$ must follow from another procedure; namely, $\mathbb{P}$ will be defined as the curl of a constraint reaction $\mathbb{L}$ (in the sense of [Fosdick and Royer-Carfagni 2004]), in appropriate function space, and due to the satisfaction of (1-1).

On the other hand, observe that the rightmost member of (1-5) has no rigorous meaning at the mesoscale since $\Lambda_{\mathscr{L}}$ is a measure and $\mathbb{P}$ a Lebesgue-integrable field.

1.4. Scope of the work. It is the goal of the present work to elucidate the functional setting allowing one to mathematically establish (1-5). To the knowledge of the authors, such a proof was nonexistent in the literature since the variational problem was unsolved until [Scala and Van Goethem 2016] at the mesoscale, in finite-strain elasticity, and for curved dislocations (i.e., loops) and dislocation networks. In this respect, it has to be emphasized that our point of view is completely different from that of [Agiasofitou and Lazar 2010], where the relation (1-5) is derived within the framework of gauge field theory of dislocations using the Noether theorem.

Considering the existence of minimizers of problem (1-3), in the present paper, we analyze the variation of $\mathscr{W}$ at the minimum points with respect to $L$, which by a formal chain rule can be written as

$$
\delta_{L} \mathscr{W}\left(F, \Lambda_{\mathscr{L}}\right)=\delta_{F} \mathcal{W}\left(F, \Lambda_{\mathscr{L}}\right) \delta_{L} F+\delta_{\Lambda} \mathcal{W}\left(F, \Lambda_{\mathscr{L}}\right) \delta_{L} \Lambda_{\mathscr{L}}
$$

Note first that $\mathcal{W}$ can be written as the sum of a deformation and a defect part, the first depending on $F$ and the second on $\Lambda_{\mathscr{L}}=-(\mathrm{Curl} F)^{\mathrm{T}}$. However, both variables are related to $L$ in an intrinsic manner, and hence, a precise meaning must be given to the above chain rule expression. 
The first aim of this paper is of a theoretical nature: basically, it consists of giving a precise meaning to $\delta_{L} F$ and $\delta_{L} \Lambda_{\mathscr{L}}$ that will be achieved by proving a series of preliminary results. As far as the second term is concerned, the geometric analysis made in [Scala and Van Goethem 2016] and synthesized in Section 3.1 is used as a basis but here completed by putting the concentrated measure $\Lambda_{\mathscr{L}}$ in duality with a certain continuous tensor, called the constraint reaction. One difficulty is related to the identification of the dual space of Radon measures which are concentrated in closed lines since in general it is not true that this set is a subspace of continuous functions. This first result will in particular require inverting the curl operator. As far as the deformation part of the energy is concerned, we have already mentioned that it is not a gradient since to satisfy constraint (1-1) it must read $F=\nabla u+\operatorname{Curl} V$ (an expression recognized as a tensor Helmholtz-Weyl-type decomposition). As a matter of fact, $F$ will depend on $L$ through the solution of $-\operatorname{Curl} \operatorname{Curl} V=$ $\Lambda_{\mathscr{L}}^{\mathrm{T}}$, which is an equation to consider with care since it is not an elliptic PDE. In this paper, use will also be made of Helmholtz- and Friedrich/Maxwell-type decompositions in $L^{p}$ (see, e.g., [Kozono and Yanagisawa 2009; Galdi 2011]), where by Maxwell these are intended estimates of vectors/tensors with respect to their curl and divergence [Neff et al. 2012b; 2012a; 2015b; Yanagisawa 2007], the crucial fact being that, by (1-1), the $L^{p}$-norm of the deformation gradient is estimated by the dislocation density norm, here intended as total variation of the Radon measure.

A direct consequence of the results in this work, discussed in Section 4.5, is setting the basis of a model of evolution in time of dislocations, in the sense that computing $\delta_{L} \mathscr{W}$ amounts to considering that a certain (configurational) force exerted on the dislocations is vanishing. Therefore, a moving dislocation will evolve with a velocity proportional to this force, as documented in dislocation theories [Hirth and Lothe 1982; Acharya 2003], and originating from the variation of the deformation part of the energy. In the final Theorem 26, we show that, at optimality, there is a balance of forces, one of which is the well-known Peach-Koehler force $\mathscr{F}$, while the other is a line-tension term, $\mathscr{G}$, provided by the variation of the defect part of the energy (see also [Conti et al. 2011]). In fact, the identity

$$
\mathscr{F}=-\mathscr{G},
$$

holding at minimality, might be considered a constitutive law for $\mathscr{F}$ since $\mathscr{G}$ is given explicitly in terms of the dislocation energy density and the line curvature and normal and tangent vectors. Let us emphasize that time evolution per se is not considered in the present work.

1.5. Structure of the paper. In Section 2, the theoretical results required as preliminaries are stated and proved, unless their proofs are found elsewhere in the 
literature. An important result is the existence of a constraint reaction, given in Section 2.4, relying on the important result on the invertibility of the curl operator as found in Section 2.3. Section 3 contains three subsections where the mathematical properties of a dislocation model in this setting are given and discussed. In particular, the functional relations between the deformation and the defect variables are given (important relations are here (3-13) and (3-14)), their admissibility is studied, and minimization results in appropriate spaces are recalled. In Section 4, the generic results of previous sections are applied to a more specific dislocation model. The goal here is to compute the first variation of the energy at the minimum points, eventually yielding the Peach-Koehler force expression in Section 4.3. In Section 4.4, a shape-optimization view of minimality provides a balance of configurational forces, which is applied to an example. All preliminary results of this paper are required to derive this force expression, collected in Theorem 26.

1.6. A remark. This paper has been written in two parts; the first, Section 2, is where all theoretical results are stated and proved without even referring to dislocations. Indeed, the functional spaces described in this section are broader than those needed for dislocations, and hence, the results more general. Instead, Sections 3 and 4 are specifically devoted to the study of dislocations, and hence, the previous statements are particularized. Moreover, in order to be self-contained, the essence of [Scala and Van Goethem 2016] is recalled in simple terms in Section 3.1.

\section{Theoretical setting and preliminary results}

2.1. Notation and conventions. The class of $3 \times 3$ matrices is denoted by $\mathbb{M}^{3}:=$ $\mathbb{R}^{3 \times 3}$. In the following definitions, the codomain space $\mathscr{R}$ is either tensor-valued, $\mathscr{R}=\mathbb{M}^{3}$, or vector valued, $\mathscr{R}=\mathbb{R}^{3}$. Then $\mathscr{R}^{\prime}$ stands for $\mathbb{R}^{3}$ or $\mathbb{R}$, respectively. The symbol $\mathcal{M}$ stands for finite Radon measures while $\mathscr{D}$ denotes the topological vector space of smooth functions with compact support. The subset of $\mathscr{R}$-valued solenoidal finite Radon measures on an open set $X \subset \mathbb{R}^{3}$ reads

$$
\mathcal{M}_{\mathrm{div}}(X, \mathscr{R}):=\left\{\mu \in \mathcal{M}(X, \mathscr{R}):\langle\mu, D \varphi\rangle=0 \text { for all } \varphi \in \mathscr{C}_{0}^{1}\left(X, \mathscr{R}^{\prime}\right)\right\},
$$

where the product (here intended in the sense of finite Radon measures) yields, in the case $\mathscr{R}=\mathbb{M}^{3}$, a real tensor whose components read $\left(\left\langle\mu_{i j}, D_{j} \varphi_{k}\right\rangle\right)_{i k}$. Recall that $\varphi \in \mathscr{C}_{0}^{1}\left(X, \mathscr{R}^{\prime}\right)$ if it is of class $\mathscr{C}^{1}$ and if for every $\epsilon>0$ there exists a compact set $K \subset X$ such that $|\varphi(x)|$ and $|D \varphi(x)|$ are smaller than $\epsilon$ for any $x \in X \backslash K$.

Observe that $\mathcal{M}_{\mathrm{div}}(X, \mathscr{R})$ is a closed subspace of $\mathcal{M}(X, \mathscr{R})$ and hence is a Banach space, endowed with the total variation norm $|\mu|(X)=\sup \{\langle\mu, \varphi\rangle: \varphi \in \mathscr{C}(X, \mathscr{R})$, $\left.\|\varphi\|_{\infty} \leq 1\right\}$ (see [Ambrosio et al. 2000] for details on vector- and tensor-valued Radon measures on metric spaces). A particular subclass of $\mathcal{M}_{\mathrm{div}}(X, \mathscr{R})$ will be 
the family of (the transpose of) the dislocation densities ${ }^{2}\left(\Lambda^{\star}\right)^{T} \in \mathcal{M}_{\mathrm{div}}\left(\widehat{\Omega}, \mathbb{M}^{3}\right)$, where $\widehat{\Omega} \supset \Omega$ is an open set containing only dislocation loops.

For a tensor $A$ and vector $N$, we use the convention $(N \times A)_{i j}=-(A \times N)_{i j}=$ $-\epsilon_{j k l} A_{i k} N_{l}$. Further, the curl of a tensor $A$ is defined componentwise as $(\operatorname{Curl} A)_{i j}=$ $\epsilon_{j k l} D_{k} A_{i l}$. As a consequence,

$$
\langle\operatorname{Curl} A, \psi\rangle=-\left\langle A_{i l}, \epsilon_{j k l} D_{k} \psi_{i j}\right\rangle=\left\langle A_{i l}, \epsilon_{l k j} D_{k} \psi_{i j}\right\rangle=\langle A, \operatorname{Curl} \psi\rangle,
$$

for every $\psi \in \mathscr{D}\left(\Omega, \mathbb{M}^{3}\right)$. In general, if $\psi$ does not have compact support,

$$
\langle\operatorname{Curl} A, \psi\rangle=\langle A, \operatorname{Curl} \psi\rangle+\int_{\partial \Omega}(N \times A) \cdot \psi d S .
$$

Note that with this convention one has $\operatorname{Div} \operatorname{Curl} A=0$ in the sense of distributions since componentwise the divergence is classically defined as (Div $A)_{i}=D_{j} A_{i j}{ }^{3}$

The following lemma characterizes the dislocation measures as a particular subclass of the solenoidal measures.

Lemma 1. Let $\mu \in M_{\mathrm{div}}\left(\Omega, \mathbb{R}^{3 \times 3}\right)$ be a measure that is absolutely continuous with respect to the $\mathscr{H}^{1}$-measure restricted on a simple Lipschitz curve $L$ with tangent vector $\tau$ and such that $L$ is either closed or ends at the boundary. Then $\mu$ is a dislocation measure; that is, there exists a constant vector $b$ such that

$$
\mu=b \otimes \tau \mathscr{H}_{\llcorner L}^{1} .
$$

We omit the proof, which is quite simple, and refer to [Scala and Van Goethem 2015]. Let us denote by $\mathcal{M}_{\text {dislo }}\left(\Omega, \mathbb{M}^{3}\right)$ the class of the transpose of such measure as $(2-4)$.

Let $1 \leq p<\infty$, and let $\Omega \subset \mathbb{R}^{3}$ be an arbitrary open set. We introduce the vector space of tensor-valued fields

$$
\mathscr{B}_{\mathscr{C}}{ }^{p}\left(\Omega, \mathbb{R}^{3 \times 3}\right):=\left\{F \in L^{p}\left(\Omega, \mathbb{R}^{3 \times 3}\right): \operatorname{Curl} F \in M_{\mathrm{div}}\left(\Omega, \mathbb{R}^{3 \times 3}\right)\right\},
$$

which, as endowed with the norm

$$
\|F\|_{\mathscr{B}^{p}}:=\|F\|_{p}+|\operatorname{Curl} F|(\Omega),
$$

turns out to be a Banach space.

${ }^{2}$ The transpose is taken to be consistent with Van Goethem's references on dislocations [Van Goethem and Dupret 2012b]. This convention was originally taken from Kröner [1981].

${ }^{3}$ In this paper, we therefore follow the transpose of Gurtin's notation convention [Cermelli and Gurtin 2001], but care must be payed since the curl and divergence of tensor fields are given alternative definitions in the literature (including [Van Goethem and Dupret 2012b; Van Goethem 2014] where the current curl would give $\operatorname{Curl} A=-A \times \nabla$ ). 
Remark 2. One might define $\mathscr{B}_{\mathscr{C}}{ }^{p}\left(\Omega, \mathbb{M}^{3}\right)$ by only specifying Curl $F \in \mathcal{M}\left(\Omega, \mathbb{M}^{3}\right)$ and considering the solenoidal property of $\mu$ as a direct consequence of the distributional identity Div Curl $F=0$ in $\Omega$.

\subsection{Helmholtz decomposition for tensor fields.}

Lemma 3. Let $G \in L^{p}\left(\Omega, \mathbb{M}^{3}\right)$ with $1<p<\infty$ and $\Omega$ be a bounded open and simply connected set with $\mathscr{C}^{1}$ boundary. There exists a unique solution (up to a constant $) \phi \in W^{1, p}\left(\Omega, \mathbb{R}^{3}\right)$ of

$$
\begin{cases}-\Delta \phi=\operatorname{Div} G & \text { in } \Omega \\ \partial_{N} \phi=-G N & \text { on } \partial \Omega .\end{cases}
$$

Moreover, such a solution satisfies $\|D \phi\|_{p} \leq C\|G\|_{p}$.

Proof. This lemma is a direct tensor extension of the theorems of existence and uniqueness of the Neumann problem as shown in [Simader and Sohr 1996] (see also [Galdi 2011, Lemma III.1.2, Theorem III.1.2]).

Note that (2-7) is a formal strong form meaning that the following weak form is solved [Yanagisawa 2007]:

$$
-\langle\nabla \phi, \nabla \varphi\rangle=\langle G, \nabla \varphi\rangle \quad \text { for all } \varphi \in W^{1, p^{\prime}}\left(\Omega, \mathbb{M}^{3}\right) .
$$

In particular, observe that the trace $G N$ is not well-defined on the domain boundary. This issue will be addressed by Lemma 4 . Let us define

$$
\begin{aligned}
L_{\text {div }}^{p}\left(\Omega, \mathbb{M}^{3}\right) & :=\left\{F \in L^{p}\left(\Omega, \mathbb{M}^{3}\right): \operatorname{Div} F=0\right\}, \\
L_{\text {curl }}^{p}\left(\Omega, \mathbb{M}^{3}\right) & :=\left\{F \in L^{p}\left(\Omega, \mathbb{M}^{3}\right): \operatorname{Curl} F=0\right\} .
\end{aligned}
$$

The following result can be proven [Van Goethem 2015].

Lemma 4. Let $\Omega \subset \mathbb{R}^{3}$ be a bounded open set with boundary of class $\mathscr{C}^{1}$, and let $F \in L^{p}\left(\Omega, \mathbb{R}^{3 \times 3}\right)$ be such that $\operatorname{Div} F \in L^{p}\left(\Omega, \mathbb{R}^{3}\right)$. Let us define the distribution $F N$ as

$$
\langle F N, \gamma(\varphi)\rangle:=\langle\operatorname{Div} F, \varphi\rangle+\langle F, D \varphi\rangle
$$

for all $\varphi \in W^{1, p^{\prime}}\left(\Omega, \mathbb{R}^{3}\right)$, with $\gamma(\varphi) \in W^{1 / p, p^{\prime}}\left(\partial \Omega, \mathbb{R}^{3}\right)$ the boundary trace of $\varphi$, where $\langle\cdot, \cdot\rangle$ always means the duality product in appropriate spaces. Then $F N \in$ $W^{-1 / p, p}\left(\partial \Omega, \mathbb{R}^{3}\right):=\left(W^{1 / p, p^{\prime}}\left(\partial \Omega, \mathbb{R}^{3}\right)\right)^{\prime}$.

Similarly, the following holds true:

Lemma 5. Let $\Omega \subset \mathbb{R}^{3}$ be a bounded open set with boundary of class $\mathscr{C}^{1}$, and let $F \in L^{p}\left(\Omega, \mathbb{R}^{3 \times 3}\right)$ be such that $\operatorname{Curl} F \in L^{p}\left(\Omega, \mathbb{R}^{3 \times 3}\right)$.

Then $F \times N \in W^{-1 / p, p}\left(\partial \Omega, \mathbb{R}^{3}\right):=\left(W^{1 / p, p^{\prime}}\left(\partial \Omega, \mathbb{R}^{3}\right)\right)^{\prime}$ is defined as

$$
\langle F \times N, \gamma(\varphi)\rangle:=\langle\operatorname{Curl} F, \varphi\rangle-\langle F, \operatorname{Curl} \varphi\rangle
$$

for all $\varphi \in W^{1, p^{\prime}}\left(\Omega, \mathbb{R}^{3}\right)$, with $\gamma(\varphi) \in W^{1 / p, p^{\prime}}\left(\partial \Omega, \mathbb{R}^{3}\right)$ the boundary trace of $\varphi$. 
Let $1<p<\infty$. In virtue of the previous two lemmas, if $V \in L^{p}\left(\Omega, \mathbb{R}^{3}\right)$ is such that $\operatorname{Div} V \in L^{p}(\Omega, \mathbb{R})$, then it is well-defined and its normal trace $V N \in$ $W^{-1 / p, p}(\partial \Omega):=\left(W^{1 / p, p^{\prime}}(\partial \Omega)\right)^{\prime}$ on $\partial \Omega$. Similarly, if $V \in L^{p}\left(\Omega, \mathbb{R}^{3}\right)$ with Curl $V \in$ $L^{p}\left(\Omega, \mathbb{R}^{3}\right)$, then its antinormal trace on $\partial \Omega$ is $V \times N \in W^{-1 / p, p}\left(\partial \Omega, \mathbb{R}^{3}\right)$ and is defined as in (2-12). These properties can be straightforwardly applied to tensorvalued maps $V \in L^{p}\left(\Omega, \mathbb{M}^{3}\right)$ so that, if $\operatorname{Div} V \in L^{p}\left(\Omega, \mathbb{R}^{3}\right)$, it is well-defined and its normal trace $V N \in W^{-1 / p, p}\left(\partial \Omega, \mathbb{R}^{3}\right)$ on $\partial \Omega$ (componentwise, $(V N)_{i}=V_{i j} N_{j}$ ). Similarly for the antinormal trace $V \times N$ (componentwise, $\epsilon_{j l p} V_{i l} N_{p}$ ), it belongs to $W^{-1 / p, p}\left(\partial \Omega, \mathbb{M}^{3}\right)$ as soon as $\operatorname{Curl} V \in L^{p}\left(\Omega, \mathbb{M}^{3}\right)$ (see also [Kozono and Yanagisawa 2009] and references therein).

Let us introduce the spaces

$$
\begin{aligned}
& \mathscr{V}^{p}(\Omega):=\left\{V \in L_{\mathrm{div}}^{p}\left(\Omega, \mathbb{M}^{3}\right): \operatorname{Curl} V \in L^{p}\left(\Omega, \mathbb{M}^{3}\right), V \times N=0 \text { on } \partial \Omega\right\}, \\
& \widetilde{\mathscr{V}}^{p}(\Omega):=\left\{V \in L_{\mathrm{div}}^{p}\left(\Omega, \mathbb{M}^{3}\right): \operatorname{Curl} V \in L^{p}\left(\Omega, \mathbb{M}^{3}\right), V N=0 \text { on } \partial \Omega\right\} .
\end{aligned}
$$

The following estimate can be found in [Kozono and Yanagisawa 2009].

Lemma 6. Let $\Omega \subset \mathbb{R}^{3}$ be a bounded open set with boundary of class $\mathscr{C}^{1}$, and assume $F \in \mathscr{V}^{p}(\Omega)$ or $F \in \widetilde{\mathscr{V}}^{p}(\Omega)$. Then $F \in W^{1, p}\left(\Omega, \mathbb{R}^{3 \times 3}\right)$, and there exists a positive constant $C=C(\Omega)$ such that

$$
\|\nabla F\|_{p} \leq C\left(\|\operatorname{Curl} F\|_{p}+\|F\|_{p}\right) .
$$

This shows that $\mathscr{V}^{p}(\Omega)$ and $\widetilde{\mathscr{V}}^{p}(\Omega)$ are closed subspaces in $W^{1, p}\left(\Omega, \mathbb{M}^{3}\right)$. By virtue of Lemma 6 and for simply connected and bounded domains, a better estimate can be found in [von Wahl 1992]. Note that the following is a classical result for smooth functions with compact support [von Wahl 1992]:

Lemma 7. Let $\Omega$ be a simply connected and bounded domain, and let $F \in \mathscr{V}^{p}(\Omega)$ or $F \in \widetilde{\mathscr{V}}^{p}(\Omega)$. Then

$$
\|\nabla F\|_{p} \leq C\|\operatorname{Curl} F\|_{p} .
$$

As a direct consequence, the following result holds.

Lemma 8. Let $\Omega$ be a simply connected and bounded domain, and let $F \in \mathscr{V}^{p}(\Omega)$ or $F \in \widetilde{\mathscr{V}}^{p}(\Omega)$. Then Curl $F=0$ if and only if $F=0$.

We remark that, when $F \in \widetilde{\mathscr{V}}^{p}(\Omega)$, Lemma 8 amounts to proving the uniqueness property of Lemma 3. Moreover, in [Kozono and Yanagisawa 2009], a more general statement is established without the simply connectedness assumption. In general, for $\Omega$ a smooth and bounded subset of $\mathbb{R}^{3}$, Curl $F=\operatorname{Div} F=0$ has a nontrivial solution. In particular, Kozono and Yanagisawa [Yanagisawa 2007] show that the solutions belong to a subspace of $\mathscr{C}^{\infty}\left(\bar{\Omega}, \mathbb{M}^{3}\right)$ with positive finite dimension, depending on the Betti numbers of $\Omega$. 
The following result is well-known in the Hilbertian case $L^{2}$ but is not classical for the general Banach space $L^{p}$. It is basically proven with the help of Lemma 3 (for a complete proof, see [Kozono and Yanagisawa 2009; Yanagisawa 2007] and also [Galdi 2011; Neff et al. 2012a]).

Theorem 9 (Helmholtz, Weyl, Hodge, and Yanagisawa). Let $1<p<\infty$, and let $\Omega$ be a bounded, simply connected, and smooth open set in $\mathbb{R}^{3}$. For every $F \in$ $L^{p}\left(\Omega, \mathbb{M}^{3}\right)$, there exist $u_{0} \in W_{0}^{1, p}\left(\Omega, \mathbb{R}^{3}\right)$ and a solenoidal $V \in \widetilde{\mathscr{V}}^{p}(\Omega)$ such that

$$
F=D u_{0}+\operatorname{Curl} V \quad\left(L^{p}\left(\Omega, \mathbb{M}^{3}\right)=\nabla W_{0}^{1, p}\left(\Omega, \mathbb{R}^{3}\right) \oplus \operatorname{Curl}^{\widetilde{V}^{p}}(\Omega)\right)
$$

Alternatively, there exist $u \in W^{1, p}\left(\Omega, \mathbb{R}^{3}\right)$ and a solenoidal $V_{0} \in \mathscr{V}^{p}(\Omega)$ such that

$$
F=D u+\operatorname{Curl} V_{0} \quad\left(L^{p}\left(\Omega, \mathbb{M}^{3}\right)=\nabla W^{1, p}\left(\Omega, \mathbb{R}^{3}\right) \oplus \operatorname{Curl}^{\mathscr{q}} \mathscr{V}^{p}(\Omega)\right) .
$$

Moreover, the decompositions are unique, in the sense that $u_{0}, V$, and $V_{0}$ are uniquely determined while $u$ is unique up to a constant, and $\left\|D u_{0}\right\|_{p},\|D u\|_{p} \leq$ $C\|F\|_{p}$, respectively.

Remark 10. When $F$ is smooth with compact support, decompositions such as (2-17) and (2-18) are classically given by the Stokes theorem and explicit formulae involving the divergence and curl of $F$ [von Wahl 1992; Bolik and von Wahl 1997].

Remark 11. Let $F$ be of class $\mathscr{C}^{1}$. In the particular case Curl $F=0$, the Helmholtz decomposition is trivial when $\Omega$ is a simply connected domain. Indeed it is wellknown that in such a case there exists $u \in \mathscr{C}^{2}\left(\Omega, \mathbb{R}^{3}\right)$ satisfying $F=D u$. This result extends for $F \in L^{p}$ with $1<p<+\infty$ as shown in [Galdi 2011]. See [Kozono and Yanagisawa 2009] for a complete treatment of Helmholtz decomposition in $L^{p}$, relying on the pioneering paper [Fujiwara and Morimoto 1977]. Moreover, if $\operatorname{Div} F=0$, then by Theorem $9, F=\operatorname{Curl} V$ with $V \in \widetilde{\mathscr{V}}^{p}(\Omega)$. Note that, for smooth functions $F$, this result holds for any simply connected domain with Lipschitz boundary.

Remark 12. Smoothness of the boundary is a strong requirement which is needed for the following reason: (2-17) and (2-18) require in principle solving a Poisson equation $\Delta u=\operatorname{Div} F$ with the right-hand side in some distributional (that is, Sobolev-Besov) space for which smoothness of the boundary is needed. It is known [Fabes et al. 1998] that for a Lipschitz boundary the solution holds for restricted $p$ (namely $\frac{3}{2}-\epsilon \leq p \leq 3+\epsilon$ ) for some $\epsilon=\epsilon(\Omega)>0$. Note that for $p=2$ a Lipschitz boundary would be sufficient.

Lemma 13. Let $\Omega \subset \mathbb{R}^{3}$ be a bounded open set with boundary of class $\mathscr{C}^{1}$, and let $V \in \mathscr{V}^{p}(\Omega)$. Then $(\operatorname{Curl} V) N=0$ in the sense of Lemma 4 . 
Proof. Take any $\varphi \in W^{1, p^{\prime}}\left(\Omega, \mathbb{R}^{3}\right)$. With by-parts integration ((2-11) and (2-12)), it holds that

$$
\langle(\operatorname{Curl} V) N, \varphi\rangle_{\partial \Omega}=\langle\operatorname{Curl} V, D \varphi\rangle=\langle V \times N, D \varphi\rangle_{\partial \Omega}=0 .
$$

Since $\varphi$ is arbitrary, the proof is done.

By Lemma 13, the function $u$ of (2-18) is found by solving (2-7) with $\phi=u$ and $G=-F$. This also gives a meaning to the condition $\partial_{N} u=F N$.

\subsection{Invertibility of the curl.}

Notation 14. Unless otherwise specified, the domains $\Omega$ we consider are bounded, smooth, and simply connected subsets of $\mathbb{R}^{3}$, with outward unit normal $N$.

Let us introduce the following notation.

Notation 15. Given $\Omega$, we denote by $\widehat{\Omega}$ another domain satisfying Notation 14 and such that $\Omega \Subset \widehat{\Omega}$.

A key equation behind the results of this work is the system

$$
\begin{cases}-\operatorname{Curl} F=\mu^{\mathrm{T}} & \text { in } \widehat{\Omega}, \\ \operatorname{Div} F=0 & \text { in } \widehat{\Omega}, \\ F N=0 & \text { on } \partial \widehat{\Omega},\end{cases}
$$

with $\mu^{\mathrm{T}}$ a Radon measure in $\mu_{\text {div }}\left(\widehat{\Omega}, \mathbb{M}^{3}\right)$. Note that the transpose is put here for convenience. In fact, the right-hand side is a general tensor-valued solenoidal bounded Radon measure. Existence and uniqueness of a solution is given by Theorem 16 below, for the proof of which Lemma 3 (or Lemma 8) will be required.

The following result is first given for general solenoidal measures and then slightly improved for dislocation measures. The existence part is a straightforward consequence of the main result of [Bourgain and Brezis 2004], whereas some further details can be found in [Scala and Van Goethem 2015, Appendix].

Theorem 16 (Biot and Savart). Let $\mu$ be a tensor-valued Radon measure such that $\mu^{\mathrm{T}} \in \mathcal{M}_{\mathrm{div}}\left(\widehat{\Omega}, \mathbb{M}^{3}\right)$. Then there exists a unique $F$ in $\mathscr{B}_{\mathrm{div}}\left(\widehat{\Omega}, \mathbb{M}^{3}\right)$ that is a solution of (2-19). Moreover, $F$ belongs to $\mathscr{B}_{\mathrm{div}}^{p}\left(\widehat{\Omega}, \mathbb{M}^{3}\right)$ for all $p$ with $1 \leq p<\frac{3}{2}$, and for all such $p$, there exists a constant $C>0$ satisfying

$$
\|F\|_{p} \leq C|\mu|(\widehat{\Omega}) .
$$

Moreover, if $\mu=\tau \otimes b \mathscr{H}_{L_{L}}^{1}$, for some $b \in \mathbb{R}^{3}$ and a $\mathscr{C}^{2}$-closed curve L in $\widehat{\Omega}$ with unit tangent vector $\tau$, then the solution $F$ belongs to $\Re_{\mathscr{C}_{\mathrm{div}}}^{p}\left(\widehat{\Omega}, \mathbb{M}^{3}\right)$ for all $p<2$.

Let us remark that the regularity assumption on the curve $L$ is necessary here since there exist examples of a measure concentrated on a rectifiable curve such 
that the associated deformation $F$ is not in $L^{p}$ with $\frac{3}{2}<p<2$, as shown in [Conti et al. 2015b].

By uniqueness, there exists a linear one-to-one and onto correspondence between the spaces $\mathcal{M}_{\mathrm{div}}\left(\widehat{\Omega}, \mathbb{M}^{3}\right)$ and $\mathscr{B} \mathscr{C}_{\mathrm{div}}^{p}\left(\widehat{\Omega}, \mathbb{M}^{3}\right)$. Thus, the map

$$
\operatorname{Curl}^{-1}: \mu_{\mathrm{div}}\left(\widehat{\Omega}, \mathbb{M}^{3}\right) \rightarrow \mathscr{B}_{\mathrm{C}} \mathscr{C}_{\mathrm{div}}^{p}\left(\widehat{\Omega}, \mathbb{M}^{3}\right), \quad v \mapsto F=-\operatorname{Curl}^{-1}(v),
$$

is well-defined and linear. Therefore, we may write

$$
\mathscr{B} \mathscr{C}_{\operatorname{div}}^{p}\left(\widehat{\Omega}, \mathbb{M}^{3}\right):=\operatorname{Curl}^{-1}\left(\mu_{\operatorname{div}}\left(\widehat{\Omega}, \mathbb{M}^{3}\right)\right) .
$$

Moreover, for any $F \in \mathscr{B} \mathscr{C}_{\text {div }}^{p}\left(\widehat{\Omega}, \mathbb{M}^{3}\right)$, we recover by (2-20) the $L^{p}$-counterpart of the Maxwell relation in $L^{2}$ [Neff et al. 2012a], that is,

$$
\|F\|_{p} \leq C|\operatorname{Curl} F|(\widehat{\Omega}) .
$$

Remark 17. In case $\Omega$ is not simply connected, the uniqueness of the solution of problem (2-19) does not hold. In such a case, Lemma 8 would also not hold since the problem might exhibit nontrivial solutions, as shown in [Yanagisawa 2007].

2.4. Existence of a constraint reaction. In the next subsections, we will deal with a linear and continuous map,

$$
\Phi: \mathscr{B} \mathscr{C}^{p}\left(\widehat{\Omega}, \mathbb{M}^{3}\right) \rightarrow \mathbb{R}
$$

such that $|\Phi(F)| \leq C\|F\|_{p}$ for some $C>0$ and satisfying

$$
L_{\text {curl }}^{p}\left(\widehat{\Omega}, \mathbb{M}^{3}\right) \subset \operatorname{ker} \Phi .
$$

An important result for maps of this kind is now stated and proved.

Theorem 18. Let $1<p<\frac{3}{2}$, and let $\Phi$ be a linear and continuous map on $L^{p}\left(\widehat{\Omega}, \mathbb{M}^{3}\right)$ satisfying $\Phi(D u)=0$ for every $u \in W^{1, p}\left(\widehat{\Omega}, \mathbb{R}^{3}\right)$. Then there exist two maps $\mathbb{L}$ and $\tilde{\mathbb{L}}$ belonging to $\mathscr{C}\left(\widehat{\widehat{\Omega}}, \mathbb{M}^{3}\right) \cap W^{1, p^{\prime}}\left(\widehat{\Omega}, \mathbb{M}^{3}\right)$, with $3<p^{\prime}<\infty$, $1 / p+1 / p^{\prime}=1$, such that, for every $F \in \mathscr{B}_{B} \mathscr{C}^{p}\left(\widehat{\Omega}, \mathbb{M}^{3}\right)$,

$$
\Phi(F)=\langle\operatorname{Curl} \tilde{\mathbb{L}}, F\rangle=\langle\operatorname{Curl} \mathbb{L}, F\rangle=\langle\mathbb{L}, \operatorname{Curl} F\rangle
$$

and satisfying $\operatorname{Div} \mathbb{L}=\operatorname{Div} \tilde{\mathbb{L}}=0$ in $\widehat{\Omega}, N \times \mathbb{L}=0$, and $\tilde{\mathbb{L}} N=0$ on $\partial \widehat{\Omega}$.

Proof. Since $\Phi$ is linear and continuous,

$$
\Phi(F)=\langle\mathbb{T}, F\rangle,
$$

for some $\mathbb{T} \in L^{p^{\prime}}\left(\widehat{\Omega}, \mathbb{M}^{3}\right)$. Now for every $\varphi \in \mathscr{C}^{\infty}\left(\widehat{\Omega}, \mathbb{R}^{3}\right)$, we have $\langle\mathbb{T}, D \varphi\rangle=$ $\Phi(D \varphi)=0$, proving that $\operatorname{Div} \mathbb{\mathbb { T }}=0$ in $\widehat{\Omega}$ and, integrating by parts, that $\mathbb{T} N=0$ on $\partial \widehat{\Omega}$. By Theorem $9((2-17)$ or $(2-18))$, there exist a unique $\mathbb{L} \in L_{\mathrm{div}}^{p^{\prime}}\left(\widehat{\Omega}, \mathbb{M}^{3}\right)$ 
satisfying $N \times \mathbb{L}=0$ on $\partial \widehat{\Omega}$ and a unique $\tilde{\mathbb{L}} \in L_{\text {div }}^{p^{\prime}}\left(\widehat{\Omega}, \mathbb{M}^{3}\right)$ with $\tilde{\mathbb{L}} N=0$ on $\partial \widehat{\Omega}$ such that

$$
\operatorname{Curl} \mathbb{L}+D u=\operatorname{Curl} \tilde{\mathbb{L}}+D u_{0}=\mathbb{T},
$$

for some $u$ and $u_{0}$ as in Theorem 9. Since Div $\mathbb{T}=0$ in $\widehat{\Omega}$, one has $u_{0}=0$ and, from Curl $\mathbb{L}=\mathbb{\mathbb { N }} N=0$ on $\partial \widehat{\Omega}, D u=0$. By the Maxwell-Friedrich-type inequality (i.e., the generalization of (2-15) [Yanagisawa 2007]), i.e.,

$$
\|\nabla \mathbb{L}\|_{p^{\prime}} \leq C\left(\|\operatorname{Curl} \mathbb{L}\|_{p^{\prime}}+\|\operatorname{Div} \mathbb{L}\|_{p^{\prime}}+\|\mathbb{L}\|_{p^{\prime}}\right),
$$

the fact that $\mathbb{L} \in L^{p^{\prime}}\left(\widehat{\Omega}, \mathbb{M}^{3}\right)$ with $\operatorname{Curl} \mathbb{L} \in L^{p^{\prime}}\left(\widehat{\Omega}, \mathbb{M}^{3}\right)$ and Div $\mathbb{L}=0$, implies that $\mathbb{L} \in W^{1, p^{\prime}}\left(\widehat{\Omega}, \mathbb{M}^{3}\right)$, which since $3<p^{\prime} \leq \infty$ entails by Sobolev embedding that

$$
\mathbb{L} \in \mathscr{C}\left(\bar{\Omega}, \mathbb{M}^{3}\right) \text {. }
$$

The same is true for $\tilde{\mathbb{L}}$. Integrating by parts the identities (2-26), we get, since $N \times \mathbb{L}=0$ on $\partial \widehat{\Omega}$,

$$
\Phi(F)=\langle\operatorname{Curl} \mathbb{L}, F\rangle=\langle\mathbb{L}, \operatorname{Curl} F\rangle,
$$

completing the proof.

In the applications, $\Phi$ will be the first variation of the deformation part of the energy. In the sequel, we will restrict to those variations whose deformation curl is concentrated in a closed curve and, specifically, is associated to some dislocation density measure. This latter notion will be made clear in Section 3.1.

\section{Energy minimization of dislocation networks}

The key point of this work is to perform variations around the minima of problem (1-3) in the largest possible functional spaces. As far as the deformation part of the energy is concerned, this amounts to proving the existence of an appropriate Lagrange multiplier to account for the constraint (1-1). This will be achieved thanks to Theorem 18. In principle, variations can be made with respect to $F$, the dislocation density $\Lambda$, and the dislocation set $L$. In the first case, one recovers the equilibrium equations, where the Piola-Kirchhoff stress is written as the curl of the constraint reaction. The second case is more delicate since the space of variations is not a linear space (due to the so-called crystallographic assumption), thus creating a series of difficulties which we do not address further. Most interesting is the variation with respect to the line, that is, with respect to infinitesimal Lipschitz variations of the optimal dislocation cluster $L^{\star}$. The difficulty here is that both $F$ and $\Lambda$ depend on $L$. In the case of $\Lambda$, the dependence is explicit since $L$ is in some sense the support of $\Lambda=\Lambda_{\mathscr{L}}$ (see (3-4)). In the case of $F$, the dependence is implicit since

$$
F=\nabla u+F^{\circ}
$$


where $F$ depends on $L$ through the relation Curl $F^{\circ}=-\left(\Lambda_{\mathscr{L}}\right)^{\mathrm{T}}$. Therefore, since the energy consists of one term in $F$ and another in $\Lambda$, variations of the energy with respect to $\mathscr{L}$ (that is, with respect to its support $L$ ) will require an appropriate version of the chain rule. This computation is the main objective of Section 4, which to be carried out carefully requires a series of preliminary steps, collected in the present section. In order to be self-contained, results from [Scala and Van Goethem 2016] are first recalled while rewritten in a concise form. We refer to [Scala and Van Goethem 2016; 2015] for a full discussion of the results and of the models. In the next two subsections, the results from Section 2 are applied to continuum dislocations. The main results are relations (3-13) and (3-14).

3.1. Dislocation density measures. In the sequel, we will adopt Notations 14 and 15. In order to perform variations in $F$ and $\Lambda$, we introduce an appropriate subspace of $\mathcal{M}_{\mathrm{div}}\left(\bar{\Omega}, \mathbb{M}^{3}\right)$ called the set of dislocation density measures and based upon the notion of integer-multiplicity (or integral) 1-currents.

In many applications, the Burgers vector is constrained by crystallographic properties to belong to a lattice. For simplicity, this lattice will be assumed isomorphic to $\mathbb{Z}^{3}$. Let the lattice basis $\left\{\bar{b}_{1}, \bar{b}_{2}, \bar{b}_{3}\right\}$ be fixed, and define the set of admissible Burgers vectors as

$$
\mathscr{B}:=\left\{b \in \mathbb{R}^{3}: \text { there exists } \beta \in \mathbb{Z}^{3} \text { such that } b=\beta_{i} \bar{b}_{i}\right\} .
$$

In the sequel, we will adopt the nonrestrictive and simple choice $\mathscr{B}=\mathbb{Z}^{3}$, i.e., $\bar{b}_{i}=e_{i}$, the $i$-th Euclidean base vector. Moreover, we write $b \in \mathbb{Z}^{3}$ to mean $b \in \mathscr{B}$.

Let $L$ be an $\mathscr{H}^{1}$-rectifiable subset of $\widehat{\Omega}, \tau$ the unit oriented tangent vector defined $\mathscr{H}^{1}$-a.e. on $L$, and $\theta: L \rightarrow \mathbb{Z}$ an $\mathscr{H}^{1}$-integrable integer-valued function. Then the integer-multiplicity 1 -current $\mathscr{L}$ denoted by $\mathscr{L}:=\{L, \tau, \theta\}$ is defined as

$$
\mathscr{L}(\omega):=\int_{L}\langle\omega, \tau\rangle \theta(x) d \mathscr{H}^{1}(x)
$$

for every compactly supported and smooth 1 -form $\omega$ defined in $\widehat{\Omega}$. The (topological vector) space of such forms is denoted by $\mathscr{D}^{1}\left(\widehat{\Omega}, \bigwedge \mathbb{R}^{3}\right)$, where $\wedge \mathbb{R}^{3}$ is the space of one-dimensional covectors.

A dislocation can be described using the notion of the integer-multiplicity 1current. For every Burgers vector $b \in \mathbb{Z}^{3}$, we introduce the regular $b$-dislocation in $\widehat{\Omega}$ as the closed integral 1-current $\widehat{\mathscr{L}}^{b}:=\left\{\hat{L}^{b}, \tau^{b}, \theta^{b}\right\}$, where $\hat{L}^{b}$ represents the union of a finite family of Lipschitz and closed curves in $\widehat{\Omega}, \tau^{b}$ its oriented unit tangent vector, and $\theta^{b}$ an integer-valued function on $\hat{L}^{b}$ called multiplicity. We define the regular $b$-dislocation $\mathscr{L}^{b}:=\left\{L^{b}, \tau^{b}, \theta^{b}\right\}$ in $\Omega$ as the restriction of $\widehat{\mathscr{L}}^{b}$ to $\bar{\Omega}$, i.e., $\mathscr{L}^{b}(\omega):=\int_{\hat{L}^{b} \cap \bar{\Omega}}\left\langle\omega, \tau^{b}\right\rangle \theta^{b}(x) d \mathscr{H}^{1}(x)$ for every compactly supported and smooth 1 -form $\omega$ defined in $\widehat{\Omega}$. Associated to any $b$-dislocation in $\widehat{\Omega}$ is its density, 
that is, the measure $\hat{\Lambda}_{\mathscr{L}^{b}} \in \mathcal{M}\left(\widehat{\Omega}, \mathbb{M}^{3}\right)$, defined by

$$
\left\langle\Lambda_{\widehat{\mathscr{L}}^{b}}, w\right\rangle:=\widehat{\mathscr{L}}^{b}\left((w b)^{*}\right),
$$

for every $w \in \mathscr{D}\left(\widehat{\Omega}, \mathbb{M}^{3}\right)$, where in the right-hand side $\omega:=(w b)^{*}$ is the covector $(w b)^{*}:=w_{k j} b_{j} d x_{k}$. If we identify test functions $w \in \mathscr{D}\left(\widehat{\Omega}, \mathbb{M}^{3}\right)$ with 1 -forms in $\mathscr{D}^{1}\left(\widehat{\Omega}, \bigwedge \mathbb{R}^{3}\right)^{3}$, then we can also identify the density $\Lambda \widehat{\mathscr{L}}_{b}$ with an integral 1-current with coefficients in the group $\mathbb{Z}^{3}$, as in (3-2). We will use the notation

$$
\Lambda_{\widehat{\mathscr{L}}^{b}}=\widehat{\mathscr{L}}^{b} \otimes b .
$$

Its counterpart in $\bar{\Omega}$ is the restriction of $\Lambda_{\widehat{\mathscr{L}}^{b}}$ to $\bar{\Omega}$, denoted by $\Lambda_{\mathscr{L}^{b}}$ and characterized by

$$
\Lambda_{\mathscr{L}^{b}}=\mathscr{L}^{b} \otimes b=\tau^{b} \otimes b \theta^{b} \mathscr{H}_{\left\llcorner L^{b}\right.}^{1} .
$$

A general dislocation $\widehat{\mathscr{L}}$ is a sequence of $b$-dislocations $\left\{\widehat{\mathscr{L}}^{b}\right\}_{b \in \mathbb{Z}^{3}}$. The associated dislocation densities in $\widehat{\Omega}$ and $\bar{\Omega}$ are given by

$$
\Lambda_{\widehat{\mathscr{L}}}=\sum_{b \in \mathbb{Z}^{3}} \Lambda_{\widehat{\mathscr{L}}^{b}} \quad \text { and } \quad \Lambda_{\mathscr{L}}=\sum_{b \in \mathbb{Z}^{3}} \Lambda_{\mathscr{L}^{b}},
$$

respectively. These definitions allow us to describe any dislocation showing a finite or countable family of Burgers vectors. However, it can be shown that actually any dislocation current $\mathscr{L}$ can be split in the basis of $\mathbb{R}^{3}$, as the sum of three integral 1-currents (called canonical dislocation currents) $\mathscr{L}=\mathscr{L}_{1}+\mathscr{L}_{2}+\mathscr{L}_{3}$, in such a way that $\Lambda_{\mathscr{L}_{i}}=\Lambda_{i}=\mathscr{L}_{i} \otimes e_{i}$ for $i=1,2,3$ and that $\Lambda_{\mathscr{L}}=\Lambda_{1}+\Lambda_{2}+\Lambda_{3}$. With the notation $\mathscr{L}_{i}=\left\{L_{i}, \tau^{i}, \theta_{i}\right\}$, we call $L:=\bigcup_{i} L_{i}$ the dislocation set, which corresponds to the support of $\mathscr{L}$ as shown in [Scala and Van Goethem 2016].

A dislocation current $\alpha$ in $V:=\widehat{\Omega} \backslash \bar{\Omega}$ is a boundary condition if it is the restriction to $V$ of a closed dislocation current $\alpha$ in $\widehat{\Omega}$. We finally define the class of admissible dislocations in $\bar{\Omega}$ with respect to a given boundary condition $\alpha$ as the set of all dislocation currents $\mathscr{L}$ which are the restrictions to $\bar{\Omega}$ of some closed dislocation current $\widehat{\mathscr{L}}$ in $\widehat{\Omega}$ such that $\widehat{\mathscr{L}}_{\llcorner V}=\alpha$. In the sequel, we will always suppose that dislocation currents are admissible for a fixed boundary datum.

3.2. Functional space representation of dislocation networks. We will restrict our attention to the class of continuum dislocations (c.d.), defined as follows: $\mathscr{L}$ is a continuum dislocation if, for $i=1,2,3$, there exists a 1-Lipschitz map $\lambda^{i}$ : $\left[0, M^{i}\right] \rightarrow \widehat{\Omega}$ such that $\widehat{\mathscr{L}}_{i}=\lambda_{\sharp}^{i} \llbracket 0, M^{i} \rrbracket$, the push-forward by $\lambda^{i}$ of the standard current given by integration on the interval $\left[0, M^{i}\right]$; see [Scala and Van Goethem $2016, \S 2$ ] for details (note that this definition is equivalent to the original one given in the reference thanks to Theorem 4.5 therein). Moreover, since all such currents are boundaryless by definition, we can rescale the functions $\lambda^{i}$ and suppose they are defined on $S^{1}$. These dislocations might be called clusters because their Lipschitz 
descriptions allow for the formation of complex curves. Their counterparts in $\bar{\Omega}$ are defined as above. In such a case, the density of a continuum dislocation in $\bar{\Omega}$ can be written as the sum of the three measures

$$
\Lambda_{\mathscr{L}}=\sum_{i=1}^{3} \Lambda_{i}=\sum_{i=1}^{3} \lambda_{\sharp}^{i} \llbracket S^{1} \rrbracket_{\llcorner\bar{\Omega}} \otimes e_{i},
$$

which can be equivalently written as $\Lambda_{i}=\left(\dot{\lambda}^{i} \otimes e_{i}\right) \lambda_{\sharp}^{i} \mathscr{H}^{1}$, where $\lambda_{\sharp}^{i} \mathscr{H}^{1}$ is the push-forward of the 1-dimensional Hausdorff measure on $S^{1}$ through $\lambda^{\vec{i}}$ (see, e.g., [Krantz and Parks 2008] for this notion).

If $\mathscr{L}$ is a continuum dislocation, then there exists a set $\mathscr{b}_{\mathscr{L}} \subset \widehat{\Omega}$ containing the support of the density $\Lambda_{\widehat{L}}$ which is a continuum, i.e., a finite union of connected compact sets with finite 1-dimensional Hausdorff measure. Note that such a set is not unique and that we can always take, for example, $\mathscr{C}_{\mathscr{L}}=\bigcup_{i=1}^{3} \lambda^{i}\left(S^{1}\right)$.

Let us introduce the class of dislocation density measures with compact support in $\widehat{\Omega}$ as

$M_{\Lambda}\left(\widehat{\Omega}, \mathbb{M}^{3}\right):=\left\{\hat{\mu} \in \mathcal{M}\left(\widehat{\Omega}, \mathbb{M}^{3}\right):\right.$ there exists $\widehat{\mathscr{L}}$, c.d., with density $\left.-\left(\Lambda_{\widehat{\mathscr{L}}}\right)^{\mathrm{T}}=\hat{\mu}\right\}$.

Let $\lambda \in W^{1,1}\left(S^{1}, \mathbb{M}^{3}\right)$, with $L:=\bigcup_{i=1}^{3} \lambda^{i}\left(S^{1}\right)$. We introduce

$\theta_{i}(P):=\#\left\{s \in\left(\lambda^{i}\right)^{-1}(P): \frac{\dot{\lambda}^{i}}{\left|\lambda^{i}\right|}(s)=\tau(P)\right\}-\#\left\{s \in\left(\lambda^{i}\right)^{-1}(P): \frac{\dot{\lambda}^{i}}{\left|\lambda^{i}\right|}(s)=-\tau(P)\right\}$,

for every $P \in L$, which stands for the multiplicity of the dislocation with Burgers vector $e_{i}$, where the symbol \# denotes the cardinality of a set (the subtraction is due to overlapping loops with reverse orientations).

For every $\varphi \in \mathscr{C}_{c}\left(\widehat{\Omega}, \mathbb{M}^{3}\right)$, the density $\hat{\mu}_{\lambda}:=-\left(\Lambda_{\widehat{L}}\right)^{\mathrm{T}}$ which is associated to $\lambda$ satisfies

$$
\begin{aligned}
-\left\langle\hat{\mu}_{\lambda}, \varphi\right\rangle & =\sum_{k=1}^{3} \int_{S^{1}} \varphi\left(\lambda^{k}(s)\right) \cdot\left(e_{k} \otimes \dot{\lambda}^{k}(s)\right) d \mathscr{H}^{1}(s) \\
& =\sum_{k=1}^{3} \int_{S^{1}}\left(\varphi \circ \lambda^{k}\right)_{k j}(s)\left(\dot{\lambda}^{k}\right)_{j}(s) d s .
\end{aligned}
$$

The latter equality can also be seen as the integration on the image $L^{i}$ of the curve $\lambda^{i}$ counted with its multiplicity $\theta_{i}$. It turns out that

$$
-\left\langle\hat{\mu}_{\lambda}, \varphi\right\rangle=\int_{L} \varphi_{i j}(P) \tau_{j}^{i}(P) \theta_{i}(P) d \mathscr{H}^{1}(P) .
$$

Here

$$
\tau_{j}^{i} \theta_{i} d \mathscr{H}^{1}=\left(\dot{\lambda}^{i}\right)_{j} d s
$$


The counterpart of $\hat{\mu}_{\lambda}$ in $\bar{\Omega}$ is $\mu_{\lambda}=\hat{\mu}_{\lambda\llcorner\bar{\Omega}}$. The correspondence between the $\operatorname{arcs} \lambda$ and the Burgers vectors of the dislocation will appear clearer in the following:

Remark 19. When we deal with a dislocation $\mathscr{L}$ generated by a single loop with Burgers vector $b=\left(\beta_{1}, \beta_{2}, \beta_{3}\right)=\beta_{i} e_{i}, \beta_{i} \in \mathbb{Z}(b \neq 0)$, then we have a Lipschitz function $\gamma^{b} \in W^{1,1}\left(S^{1}, \mathbb{R}^{3}\right)$ such that $\mathscr{L}=\gamma_{\sharp}^{b} \llbracket S^{1} \rrbracket_{\llcorner\bar{\Omega}}$ and $-\mu_{\gamma^{b}}^{\mathrm{T}}=\Lambda_{\mathscr{L}}=\mathscr{L} \otimes b$, that is, the measure such that

$$
\begin{aligned}
-\left\langle\mu_{\gamma^{b}}, \varphi\right\rangle & =\int_{S^{1}} \varphi\left(\gamma^{b}(s)\right) \cdot\left(b \otimes \dot{\gamma}^{b}(s)\right) d s=\int_{S^{1}} \varphi_{i j}\left(\gamma^{b}(s)\right) b_{i} \dot{\gamma}_{j}^{b}(s) d s \\
& =\int_{L} \varphi_{i j} \tau_{j}^{i} b_{i} \theta d \mathscr{H}^{1},
\end{aligned}
$$

where $\theta(P)$ represents the multiplicity of the dislocation and is defined for every $P \in L$ as

$\theta(P):=\#\left\{s \in\left(\gamma^{b}\right)^{-1}(P): \frac{\dot{\gamma}^{b}}{\left|\gamma^{b}\right|}(s)=\tau(P)\right\}-\#\left\{s \in\left(\gamma^{b}\right)^{-1}(P): \frac{\dot{\gamma}^{b}}{\left|\gamma^{b}\right|}(s)=-\tau(P)\right\}$.

For every $\mu \in M_{\Lambda}\left(\widehat{\Omega}, \mathbb{M}^{3}\right)$, it is easy to check that Div $\mu=0$ in $\widehat{\Omega}$ since $\mathscr{L}_{i}$ are closed integral currents. In fact for all $\psi \in \mathscr{D}\left(\widehat{\Omega}, \mathbb{R}^{3}\right)$, one has

$$
\begin{aligned}
-\langle D \psi, \mu\rangle=\left\langle D \psi, \sum_{k=1}^{3} e_{k} \otimes \dot{\lambda}^{k}\left(\lambda_{\sharp}^{k} \mathcal{H}^{1}\right)\right\rangle=\sum_{i=1}^{3} \int_{S^{1}} D_{j}\left(\psi_{i} \circ \lambda^{i}\right) \dot{\lambda}_{j}^{i} d s & \\
& =\int_{S^{1}} D_{t}\left(\psi_{k} \circ \lambda^{k}\right) d t=0 .
\end{aligned}
$$

We then get $\mu_{\Lambda}\left(\widehat{\Omega}, \mathbb{M}^{3}\right) \subset M_{\text {div }}\left(\widehat{\Omega}, \mathbb{M}^{3}\right)$. We can now identify the space $\mu_{\Lambda}\left(\widehat{\Omega}, \mathbb{M}^{3}\right)$ with $W^{1,1}\left(S^{1}, \widehat{\Omega}^{3}\right)$ through the map

$$
T: W^{1,1}\left(S^{1}, \widehat{\Omega}^{3}\right) \rightarrow M_{\Lambda}\left(\widehat{\Omega}, \mathbb{M}^{3}\right) \text { such that } T(\lambda)=-\hat{\mu}_{\lambda} \text { defined in (3-6). }
$$

The map $T$ is by definition onto while, for every $\lambda \in W^{1,1}\left(S^{1}, \widehat{\Omega}^{3}\right)$,

$$
\|T(\lambda)\|_{\mathcal{M}} \leq\|\dot{\lambda}\|_{L^{1}}
$$

implying the continuity of $T$. In general, $T$ is not an injective map, but it is injective up to an equivalence relation $\sim$ in $W^{1,1}\left(S^{1}, \widehat{\Omega}^{3}\right.$ ) (namely, $\lambda \sim \lambda^{\prime}$ if and only if $T(\lambda)=T\left(\lambda^{\prime}\right)$ as measures). As a consequence,

$$
\begin{aligned}
T\left(W^{1,1}\left(S^{1}, \widehat{\Omega}^{3}\right)\right) & =M_{\Lambda}\left(\widehat{\Omega}, \mathbb{M}^{3}\right), \\
T^{-1}\left(M_{\Lambda}\left(\widehat{\Omega}, \mathbb{M}^{3}\right)\right) & =W^{1,1}\left(S^{1}, \widehat{\Omega}^{3}\right) .
\end{aligned}
$$


3.3. Class of admissible deformations and existence of minimizers. In this subsection, we exhibit an existence result for minimizers of energies $W$ satisfying some particular assumptions. For the proofs, we refer to [Scala and Van Goethem 2016]. Let us introduce

$$
\begin{aligned}
& \mathscr{B}_{\mathscr{C}}{ }^{p, \Lambda}\left(\widehat{\Omega}, \mathbb{M}^{3}\right):=\left\{F \in \mathscr{B}^{p}\left(\widehat{\Omega}, \mathbb{M}^{3}\right): \operatorname{Curl} F \in M_{\Lambda}\left(\widehat{\Omega}, \mathbb{M}^{3}\right)\right\} \\
& \mathscr{B}_{\mathscr{C}}{ }^{p, \Lambda}\left(\Omega, \mathbb{M}^{3}\right):=\left\{F \in \mathscr{B}^{p}\left(\Omega, \mathbb{M}^{3}\right)\right. \\
& \text { : there exists } \left.\widehat{F} \in \mathscr{B}_{B} \mathscr{C}^{p, \Lambda}\left(\widehat{\Omega}, \mathbb{M}^{3}\right) \text { with } F=\widehat{F}_{\llcorner\Omega}\right\}
\end{aligned}
$$

and its proper subspace

$$
\mathscr{B} \mathscr{C}_{\operatorname{div}}^{p, \Lambda}\left(\widehat{\Omega}, \mathbb{M}^{3}\right):=\left\{F \in \mathscr{B} \mathscr{C}_{\operatorname{div}}^{p}\left(\widehat{\Omega}, \mathbb{M}^{3}\right): \operatorname{Curl} F \in \mathcal{M}_{\Lambda}\left(\widehat{\Omega}, \mathbb{M}^{3}\right)\right\}
$$

in such a way that, by Theorem 16 and (3-13),

$$
\mathscr{B}_{\operatorname{div}}^{p, \Lambda}\left(\widehat{\Omega}, \mathbb{M}^{3}\right):=\operatorname{Curl}^{-1}\left(M_{\Lambda}\left(\widehat{\Omega}, \mathbb{M}^{3}\right)\right)=\operatorname{Curl}^{-1}\left(T\left(W^{1,1}\left(S^{1}, \widehat{\Omega}^{3}\right)\right)\right) .
$$

In [Scala and Van Goethem 2016], we consider deformations $F \in \mathscr{B} \mathscr{C}^{p, \Lambda}\left(\Omega, \mathbb{M}^{3}\right)$ which also satisfy some regularity conditions outside the continuum dislocation set $\mathscr{C}_{\mathscr{L}}$ of the dislocation $\Lambda_{\mathscr{L}} \in \mathcal{M}_{\Lambda}\left(\bar{\Omega}, \mathbb{M}^{3}\right)$. If $F$ is an admissible deformation, we assume that $F$ satisfies the following property:

(P) For every ball $B \subset \Omega$ with $B \cap \mathscr{C}_{\mathscr{L}}=\varnothing$, there exists a Cartesian map $u \in$ $\operatorname{Cart}^{p}\left(B, \mathbb{R}^{3}\right)$ such that $F=D u$ in $B$.

Let us recall the meaning of $\operatorname{Cart}^{p}\left(B, \mathbb{R}^{3}\right)$. If $U$ is an open set on $\mathbb{R}^{3}$, the space of Cartesian maps on $U$, denoted by $\operatorname{Cart}^{p}\left(U, \mathbb{R}^{3}\right)$, is defined as the space of maps $u: U \rightarrow \mathbb{R}^{3}$ belonging to $W^{1, p}\left(U, \mathbb{R}^{3}\right)$ and satisfying the conditions that $\operatorname{adj}(D u)$ and $\operatorname{det}(D u)$ belong to $L^{1}\left(U, \mathbb{M}^{3}\right)$ and $\partial \mathscr{G}_{u}=0$, where $\mathscr{G}_{u}$ is the rectifiable 3-current in $U \times \mathbb{R}^{3}$ carried by the graph of $u$ [Giaquinta et al. 1998]. We denote by

$$
\begin{aligned}
& \mathscr{A D}^{p}(\widehat{\Omega}):=\left\{F \in \mathscr{B} \mathscr{C}^{p, \Lambda}\left(\widehat{\Omega}, \mathbb{M}^{3}\right): F \text { satisfies }(\mathrm{P}) \text { above }\right\} \\
& \mathscr{A D}^{p}(\Omega):=\left\{F \in \mathscr{B}^{p} \mathscr{C}^{p}\left(\Omega, \mathbb{M}^{3}\right): \text { there exists } \widehat{F} \in \mathscr{A}^{p}(\widehat{\Omega}) \text { with } F=\widehat{F}_{\llcorner\Omega}\right\} .
\end{aligned}
$$

Notation 20. Let $\widehat{\Omega}$ be the open set introduced in Notation 15, and let $\alpha$ be a boundary condition in $V=\widehat{\Omega} \backslash \bar{\Omega}$ (i.e., $\alpha=\widehat{\mathscr{L}}_{\llcorner V}$ for a closed dislocation current $\widehat{\mathscr{L}}$ in $\widehat{\Omega})$. We then fix $\widehat{F} \in \mathscr{A}_{\mathscr{D}^{p}}(\widehat{\Omega})$ such that $-\operatorname{Curl} \widehat{F}=\left(\Lambda_{\widehat{L}}\right)^{\mathrm{T}}$ and define

$$
\begin{aligned}
\mathscr{F}_{\alpha}:=\left\{F \in \mathscr{A}^{p}(\Omega),\right. & 1 \leq p<2: \widetilde{F}:=F \chi_{\Omega}+\widehat{F} \chi_{V} \in \mathscr{A}^{p}(\widehat{\Omega}),-\operatorname{Curl} \widetilde{F}=\left(\Lambda_{\widehat{\mathscr{L}}}\right)^{\mathrm{T}} \\
& \text { in } \widehat{\Omega} \text { for some closed dislocation current } \widehat{\mathscr{L}} \text { in } \widehat{\Omega}\} . \quad(3-21)
\end{aligned}
$$

In particular, note that the dislocation current $\widehat{\mathscr{L}}$ in the above definition must coincide with $\alpha$ in $V$. We denote by $\mathscr{L}$ the restriction to $\bar{\Omega}$ of $\widehat{\mathscr{L}}$. 
Assumptions on the energy. We make the assumption on the elastic energy

$$
\mathscr{W}\left(F, \Lambda_{\mathscr{L}}\right):=\mathcal{W}_{\mathrm{e}}(F)+\mathcal{W}_{\text {dislo }}\left(\Lambda_{\mathscr{L}}\right)
$$

with

$$
W_{\mathrm{e}}(F):=\int_{\Omega} W_{\mathrm{e}}(F) d x
$$

As for the dislocation part, we assume that

$$
\mathcal{W}_{\text {dislo }}\left(\Lambda_{\mathscr{L}}\right)=\mathcal{W}_{\text {dislo }}^{1}\left(\Lambda_{\mathscr{L}}\right)+\mathcal{W}_{\text {dislo }}^{2}\left(\Lambda_{\mathscr{L}}\right)
$$

where the precise continuity, growth properties on the bulk, and defect energies are discussed and motivated in [Scala and Van Goethem 2016]. Let us stress that following [Conti et al. 2015a] (where no variational problem is solved), an expression for the line tension $W_{\text {dislo }}^{1}$ is here taken as

$$
\mathcal{W}_{\text {dislo }}^{1}(\mu)=\int_{L} \psi(\theta b, \tau) d \mathscr{H}^{1}
$$

when $\mu=b \otimes \gamma_{\sharp} \llbracket\left[S^{1} \rrbracket=b \otimes \theta \tau \mathscr{H}_{\llcorner L}^{1}\right.$ is the dislocation density of a cluster generated by the loop $\gamma \in W^{1,1}\left(S^{1}, \mathbb{R}^{3}\right)$ and Burgers vector $b=\beta_{i} e_{i}, \beta_{i} \in \mathbb{Z}(b \neq 0)$, and takes the value $+\infty$ if $\mu$ is not of this type. Here $\psi: \mathbb{Z}^{3} \times \mathbb{R}^{3} \rightarrow \mathbb{R}$ is a nonnegative function satisfying $\psi(0, \cdot)=0$ and $\psi(b, t) \geq c\|b\|$ for a constant $c>0$.

As for the term $\mathcal{W}_{\text {dislo }}^{2}\left(\Lambda_{\mathscr{L}}\right)$, it is remarkable that, under the hypotheses needed to get existence of minimizers, it does not depend on small perturbations of the dislocation line set $L$. This will be strongly used in the subsequent section.

Now the existence theorem is the following:

Theorem 21. Under Notation 20 and suitable hypotheses on the energy $W$ in (3-22) (see [Scala and Van Goethem 2016] for details), there exists a minimizer $F^{\star}$ of the problem

$$
\min _{F \in \mathscr{F}_{\alpha}} \mathscr{W}\left(F, \Lambda_{\mathscr{L}}\right)
$$

We write Curl $F^{\star}=\Lambda_{\mathscr{L}^{\star}}^{\mathrm{T}}$ with $\mathscr{L}^{\star}$ being the optimal dislocation network, whose support is denoted by $L^{\star}$. It should be remarked that, due to the Dirichlet condition $F=\widehat{F}$ on $\widehat{\Omega} \backslash \Omega$ for the admissible deformations gradients, the minimizer is not trivial and must satisfy $-\operatorname{Curl} F=\Lambda_{\mathscr{L}}$ for some closed dislocation current $\mathscr{L}$ coinciding with $\alpha$ in $\widehat{\Omega} \backslash \Omega$. An explicit example showing the nontriviality of the solution can be found in [Scala and Van Goethem 2016, §5.4]. Note that such energies at the macroscale are considered in [Neff et al. 2015a], where a variational problem is solved. 


\section{Configurational forces at optimal dislocation networks}

Certain forces apply on the dislocation clusters, solutions to the above minimization problem. They are due to the combined effect of the deformation and defect part of the energy. The line having no mass, these forces must be understood as being of configurational nature. They are related to the presence of microstructure, here dislocations, in an otherwise static elastic medium in equilibrium. All the results of the previous sections will allow us to prove Theorem 26, which consists of a balance of forces at minimality. Furthermore, minimality will entail EulerLagrange equations which physically correspond to the balance of forces and to the vanishing of virtual work done by the configurational force, recognized as the Peach-Koehler force.

4.1. Shape variation at optimality. Let $F^{\star}$ be a minimizer of $\mathscr{W}(F)$. By Theorem 9 and (3-13),

$$
F^{\star}=D u^{\star}+\left(\operatorname{Curl}^{-1} \circ T\right)\left(\lambda^{\star}\right)
$$

where $\mathrm{Curl}^{-1}$ is the solution of (2-19), for some $\lambda^{\star} \in W^{1,1}\left(S^{1}, \widehat{\Omega}^{3}\right)$. Let $-\left(\Lambda^{\star}\right)^{\mathrm{T}}:=$ $T\left(\lambda^{\star}\right)=-\operatorname{Curl} F^{\star}$ on $\widehat{\Omega}$.

Define the linear map

$$
S: W^{1,1}\left(S^{1}, \widehat{\Omega}^{3}\right) \rightarrow \mathscr{B}_{\operatorname{div}}^{p, \Lambda}\left(\widehat{\Omega}, \mathbb{M}^{3}\right), \quad S=\operatorname{Curl}^{-1} \circ T .
$$

We first prove the following preliminary result.

Lemma 22. The map $S: W^{1,1}\left(S^{1}, \widehat{\Omega}^{3}\right) \rightarrow \mathscr{B} \mathscr{C}_{\text {div }}^{p, \Lambda}\left(\widehat{\Omega}, \mathbb{M}^{3}\right)$ is Gâteaux differentiable at $\lambda^{\star}$ in all directions $\lambda$. In particular, $D S\left(\lambda^{\star}\right)[\lambda] \in \mathcal{M}\left(\widehat{\Omega}, \mathbb{M}^{3}\right)$, for every $W^{1,1}\left(S^{1},\left(\mathbb{R}^{3}\right)^{3}\right)$-variation $\lambda$, and

$$
\left\langle D S\left(\lambda^{\star}\right)[\lambda], \varphi\right\rangle=\sum_{i=1}^{3} \int_{S^{1}} \epsilon_{j k m}\left(\varphi \circ \lambda^{\star}\right)_{i m}(s)\left(\dot{\lambda}^{\star}\right)_{k}^{i}(s) \lambda_{j}^{i}(s) d s
$$

for every $\varphi \in \mathscr{C}_{c}\left(\Omega, \mathbb{M}^{3}\right)$ such that $\operatorname{Div} \varphi=0$.

Proof. Let $\Psi \in \mathscr{D}\left(\Omega, \mathbb{M}^{3}\right)$. From (3-6) and (3-11), we infer by a Taylor expansion of $\Psi$ that the directional derivative of $T$ at $\lambda^{\star}$ along a variation $\lambda \in W^{1,1}\left(S^{1},\left(\mathbb{R}^{3}\right)^{3}\right)$ reads

$$
\left\langle D T\left(\lambda^{\star}\right)[\lambda], \Psi\right\rangle=\sum_{i=1}^{3} \int_{S^{1}}\left(\left(\Psi \circ \lambda^{\star}\right)_{i j}(s) \dot{\lambda}_{j}^{i}(s)+D_{k}\left(\Psi \circ \lambda^{\star}\right)_{i j}(s)\left(\dot{\lambda}^{\star}\right)_{j}^{i}(s) \lambda_{k}^{i}(s)\right) d s .
$$


Integrating the last expression by parts, we get

$$
\begin{aligned}
\left\langle D T\left(\lambda^{\star}\right)[\lambda], \Psi\right\rangle= & \sum_{i=1}^{3} \int_{S^{1}}\left(D_{k}\left(\Psi \circ \lambda^{\star}\right)_{i j}(s)\left(\dot{\lambda}^{\star}\right)_{j}^{i}(s) \lambda_{k}^{i}(s)\right. \\
& \left.-D_{k}\left(\Psi \circ \lambda^{\star}\right)_{i j}(s)\left(\dot{\lambda}^{\star}\right)_{k}^{i}(s) \lambda_{j}^{i}(s)\right) d s \\
= & \sum_{i=1}^{3} \int_{S^{1}}\left(D_{j}\left(\Psi \circ \lambda^{\star}\right)_{i k}(s)-D_{k}\left(\Psi \circ \lambda^{\star}\right)_{i j}(s)\right)\left(\dot{\lambda}^{\star}\right)_{k}^{i}(s) \lambda_{j}^{i}(s) d s \\
= & \sum_{i=1}^{3} \int_{S^{1}} \epsilon_{j k m} \epsilon_{m p q} D_{p}\left(\Psi \circ \lambda^{\star}\right)_{i q}(s)\left(\dot{\lambda}^{\star}\right)_{k}^{i}(s) \lambda_{j}^{i}(s) d s .
\end{aligned}
$$

Let us now compute $D S$. Let $\varphi \in \mathscr{C}_{c}\left(\Omega, \mathbb{M}^{3}\right)$ such that $\operatorname{Div} \varphi=0$. Then $\varphi=\operatorname{Curl} \Psi$ for some $\Psi \in C^{1}\left(\Omega, \mathbb{M}^{3}\right)$, and hence, by Theorem 16 ,

$$
\begin{aligned}
\frac{1}{\epsilon}\left\langle S\left(\lambda^{\star}+\epsilon \lambda\right)-S\left(\lambda^{\star}\right), \operatorname{Curl} \Psi\right\rangle & =\frac{1}{\epsilon}\left\langle\operatorname{Curl}^{-1}\left(T\left(\lambda^{\star}+\epsilon \lambda\right)-T\left(\lambda^{\star}\right)\right), \operatorname{Curl} \Psi\right\rangle \\
& =\frac{1}{\epsilon}\left\langle\left(T\left(\lambda^{\star}+\epsilon \lambda\right)-T\left(\lambda^{\star}\right)\right), \Psi\right\rangle .
\end{aligned}
$$

Letting $\epsilon \rightarrow 0$ yields the result by (4-3).

4.2. First Euler-Lagrange equation and the static equilibrium. In this subsection, we make variations of the deformation at minimality, assuming the optimal line fixed, and derive the classical strong form of finite-strain elasticity.

Regularity assumption on the energy. We make the assumption that the energy $W_{\mathrm{e}}: L^{p}(\widehat{\Omega}) \rightarrow \mathbb{R}$ in (3-23) is Fréchet differentiable in $L^{p}(\widehat{\Omega})$ with the Fréchet derivative of $F \mapsto \mathscr{W}\left(F, \Lambda^{\star}\right)$ denoted by $W_{F} \in L^{p^{\prime}}(\widehat{\Omega})$. As a consequence, for every $F \in L^{p}(\widehat{\Omega})$,

$\left(\mathrm{A}_{1}\right) \delta \mathcal{W}\left(F^{\star}\right)[F]:=\frac{d}{d \epsilon} \mathscr{W}\left(F^{\star}+\epsilon F, \Lambda^{\star}\right)_{\mid \epsilon=0}=\int_{\widehat{\Omega}} W_{F}^{\star} \cdot F d x=\delta W_{\mathrm{e}}\left(F^{\star}\right)[F]$ with $W_{F}^{\star}:=W_{F}\left(F^{\star}, \Lambda^{\star}\right)=\delta W_{\mathrm{e}}\left(F^{\star}\right) \in L^{p^{\prime}}(\widehat{\Omega})$.

Note that this assumption is rather general and is about the least we can assume on $\mathcal{W}_{\mathrm{e}}$.

Variations $F$ of deformation $F^{\star}$ still satisfying the constraint $-\operatorname{Curl}\left(F^{\star}+\epsilon F\right)=$

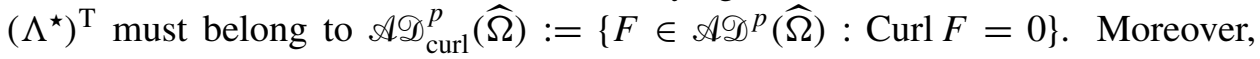
such variations at the minimum points of the energy $\mathscr{W}$ must provide a vanishing variation of $\mathcal{W}$. Thus, $F^{\star}$ being such a solution, for every curl-free $F=D u \in$ $L^{p}(\widehat{\Omega})$, it must hold that

$$
\delta W^{\star}(D u)=\delta W_{\mathrm{e}}\left(F^{\star}\right)[D u]=0
$$


From $\left(\mathrm{A}_{1}\right),(4-4)$, and Theorem 18 , it follows that there exists $\mathbb{\Perp}^{\star}$ continuous such that

$$
\mathbb{P}^{\star}:=W_{F}^{\star}=\delta W_{\mathrm{e}}\left(F^{\star}\right)=\operatorname{Curl} \mathbb{L}^{\star} \in L^{p^{\prime}}(\widehat{\Omega})
$$

satisfying the strong form

$$
\begin{cases}-\operatorname{Div} \mathbb{P}^{\star}=0 & \text { in } \widehat{\Omega}, \\ \mathbb{P}^{\star} N=0 & \text { on } \partial \widehat{\Omega} .\end{cases}
$$

One could wonder why (4-6) is not immediate from the relation $\int_{\Omega} W_{F}^{\star} \cdot \nabla u d x=0$, which is Euler-Lagrange in weak form. In fact, the integration by parts which is classically used in this context is not legitimate in the present case simply because the divergence theorem does not hold for $L^{p}$-fields since $\mathbb{P}^{\star} N$ has no meaning at the boundary. This is the reason why Theorem 18 is called and $\mathbb{P}^{\star}$ is obtained as the curl of constraint reaction $\mathbb{L}^{\star}$, being therefore automatically divergence-free, while $\mathbb{P}^{\star} N$ has a meaning by Lemma 13 and Theorem 18 .

Remark 23. By (4-6), $\mathbb{P}^{\star}:=W_{F}^{\star}$ is identified with the first Piola-Kirchhoff stress. $\mathbb{P}^{\star}$ being in $L^{p^{\prime}}(\widehat{\Omega})$, and recalling that $F \in L^{p}(\widehat{\Omega})$, means that the Kirchhoff stress $\mathbb{P}^{\star} F$ is in $L^{1}(\widehat{\Omega})$.

4.3. The Peach-Koehler force as a stationary condition. In this subsection, we derive the second Euler-Lagrange equation of the system in equilibrium.

Regularity assumption on the stress. Regularity of the minimizers is a well-known open problem in mathematical elasticity. Indeed, almost no results exist, even with an energy growth with $p \geq 2$ (i.e., without dislocations), as reported by J. Ball [2002]. A related problem is the regularity of the Piola-Kirchhoff stress $\mathbb{P}^{\star}$. In order to derive the subsequent formulae, which are well-established by physicists, we will also appeal to an assumption, not on $F^{\star}$, but rather on some components of $\mathbb{P}^{\star}$.

Let us consider the orthonormal curvilinear basis $\left(\tau^{\star}, \sigma^{\star}, v^{\star}\right)$ on the optimal dislocation set $L^{\star}$, with $\tau^{\star}$ the unit tangent vector to $L^{\star}$. Let us decompose $\mathbb{P}^{\star}$ in this basis; i.e., $\mathbb{P}^{\star}=\mathbb{P}^{\star} \tau^{\star} \otimes \tau^{\star}+\widetilde{\mathbb{P}}^{\star}$. Physically, $\mathbb{P}^{\star} \tau^{\star}$ represents the force $d \mathscr{F}$ exerted on a facet $d S$ of normal $\tau^{\star}$, that is, on a section of the tubular neighborhood of the dislocation $L^{\star}$; namely, $d \mathscr{F}=\mathbb{P}^{\star} \tau^{\star} d S$. Since all such facets are crossed by the dislocation, they presumably correspond to singular forces, in such a way that no regularity assumption can be made on these components. We will therefore make a regularity assumption on the remaining components $\widetilde{\mathbb{P}}^{\star}$. Let us emphasize that the optimal deformation tensor $F^{\star}$ is smooth in $\Omega \backslash L^{\star}$, and hence, by (4-6), $\mathbb{P}^{\star}$ will also be smooth in $\Omega \backslash L^{\star}$. Therefore, it is assumed that

$\left(\mathrm{A}_{2}\right) \widetilde{\mathbb{P}}^{\star}$ is continuous in a neighborhood of $L^{\star}$.

In fact, lack of continuity of these components would mean that the contact forces $d \mathscr{F}$ tend to infinity at $L^{\star}$. 
Validity of Assumption $\left(\mathrm{A}_{2}\right)$. First, we remark that in linearized elasticity the stress behaves as $\sim 1 / r$ and following [Hirth and Lothe 1982; Van Goethem and Dupret 2012a] one has $\widetilde{\mathbb{P}}_{\text {screw }}^{\star}=0$ whereas $\widetilde{\mathbb{P}}_{\text {edge }}^{\star} \rightarrow \infty$ as $r \rightarrow 0$. Hence, obviously, one must consider finite strain elasticity to discuss Assumption $\left(\mathrm{A}_{2}\right)$. Here again, the situation is not evident since nonlinear stresses depend on the choice of the material (i.e., of the energy $W_{\mathrm{e}}$ ) and on the physics which takes place at the singular line. We will thus follow L. Zubov, who has reported the current state of the art in [Zubov 1997]. About the screw dislocation, he first points out that, for a Mooney (and neo-Hookean) incompressible material, one has divergence of $\widetilde{\mathbb{P}}_{\text {screw }}^{\star}$ at the singularity, but he discards this case as being nonphysical, i.e., not suitable for the creation of a screw dislocation [Zubov 1997, p. 74]. Then he considers the Bartenev-Khazanovich among two other incompressible materials and finds $\widetilde{\mathbb{P}}_{\text {screw }}^{\star} \sim \ln r$, hence again lacking continuity though showing better integrability properties. However, incompressibility is not assumed in general and in particular not in the present paper (indeed, it would imply another constraint reaction [Fosdick and Royer-Carfagni 2005]). Therefore, following Zubov again, one considers a Blatz-Ko material together with the physical observation that the creation of a screw dislocation takes place together with a cylindrical cavity, and this implies continuity of $\widetilde{\mathbb{P}}_{\text {screw }}^{\star}$ at the singularity [Zubov 1997, (3.2.12), (3.2.16), (3.2.24), (3.2.28), p. 76], whereas $\mathbb{P}-\widetilde{\mathbb{P}}$ must not be continuous [Zubov 1997, (3.2.12), p. 76]. Because the technical difficulties are huge, Zubov does not compute in extenso the edge dislocation with a cavitation, but nonetheless we consider the following physical interpretation, as based on Zubov's aforementioned results and physical evidence of dislocation nucleation as reported by, e.g., [Cottrell 1964; Berezhkova 1969]:

Assumption $\left(\mathrm{A}_{2}\right)$ holds true for a compressible material where a cavitation is found along any dislocation loop.

In practice, Assumption $\left(\mathrm{A}_{2}\right)$ allows one to have a finite radius $R$ in the reference configuration corresponding to $r=0$ in the deformed configuration $\Omega$. Furthermore, Zubov shows that $R(0)$ is proportional, on the order of $10 \%$ of the Burgers vector.

Note that the creation of such a cavity in single crystals is due to the nucleation process of dislocation loops resulting from the collapse of a void, i.e., a cluster of vacancies which has become unstable.

The Peach-Koehler force. We write $F=D u+\operatorname{Curl}^{-1}\left(-\Lambda^{\mathrm{T}}\right)=D u+S(\lambda)$ for all $F \in \mathscr{B}_{\mathfrak{C}}{ }^{p}\left(\widehat{\Omega}, \mathbb{R}^{3 \times 3}\right)$ with $u \in W^{1, p}\left(\widehat{\Omega}, \mathbb{R}^{3}\right)$ (by Theorem 9 and (4-1)). Following this formalism, it is thus assumed that the energy $\mathscr{W}$ depends on the dislocation path $\lambda \in W^{1,1}\left(S^{1}, \widehat{\Omega}^{3}\right)$ as defined in (3-11), that is,

$$
W^{\circ}(D u, \lambda):=\mathscr{W}(D u+S(\lambda),-T(\lambda)),
$$


and then $\mathscr{W}^{\circ}(D u, \lambda)=\mathscr{W}(F, \Lambda)$ with $\Lambda^{\mathrm{T}}=-\operatorname{Curl} F$ if $\lambda \in T^{-1}\left(-\Lambda^{\mathrm{T}}\right)$. Let us consider the energy at its minimum $F^{\star}$ :

$$
\begin{aligned}
\mathscr{W}^{\circ}\left(D u^{\star}, \lambda^{\star}\right) & :=\mathscr{W}\left(D u^{\star}+S\left(\lambda^{\star}\right),-T\left(\lambda^{\star}\right)\right) \\
& =\mathscr{W}_{\mathrm{e}}\left(D u^{\star}+S\left(\lambda^{\star}\right)\right)+\mathscr{W}_{\text {defect }}\left(-T\left(\lambda^{\star}\right)\right) \\
& =: \mathscr{W}_{\mathrm{e}}^{\circ}\left(\lambda^{\star}\right)+\mathscr{W}_{\text {defect }}^{\circ}\left(\lambda^{\star}\right) .
\end{aligned}
$$

Let us denote the variation of the energy $\mathcal{W}_{\mathrm{e}}$ by $\delta^{\circ} \mathcal{W}_{\mathrm{e}}\left(F^{\star}\right):=\delta \mathcal{W}_{\mathrm{e}}^{\circ}\left(\lambda^{\star}\right)$. The expression of the variation of the energy is then given by the following main result.

Theorem 24 (work done by the Peach-Koehler force). Under the assumptions of Theorem 21 and hypotheses $\left(\mathrm{A}_{1}\right)$ and $\left(\mathrm{A}_{2}\right)$, one has

$$
\left\langle\delta^{\circ} W_{\mathrm{e}}\left(F^{\star}\right), \lambda\right\rangle=\sum_{i=1}^{3} \int_{L^{\star}}\left(\mathbb{P}^{\star} \times \tau^{i}\right)^{\mathrm{T}} \theta_{i} \cdot \lambda^{i} \circ\left(\lambda^{\star}\right)^{-1} d \mathcal{H}^{1},
$$

where we have employed notation (3-7).

Proof. We want to perform variations $\lambda \in W^{1,1}\left(S^{1},\left(\mathbb{R}^{3}\right)^{3}\right)$ of $\mathscr{W}_{\mathrm{e}}^{\circ}\left(\lambda^{\star}\right)$. Identifying $W_{F}^{\star}$ with the Piola-Kirchhoff $\mathbb{P}^{\star}$ as in (4-5), using Lemma 22 with $\varphi=\widetilde{\mathbb{P}}$, one has

$$
\begin{aligned}
\delta W_{\mathrm{e}}^{\circ}\left(\lambda^{\star}\right)[\lambda] & =\sum_{i=1}^{3} \int_{S^{1}} \lambda_{j}^{i} \epsilon_{j k m}\left(\dot{\lambda}^{\star}\right)_{k}^{i} \widetilde{\mathbb{P}}_{i m}^{\star} \circ \lambda^{\star} d s \\
& =\sum_{i=1}^{3} \int_{S^{1}} \lambda_{j}^{i} \epsilon_{j k m}\left(\dot{\lambda}^{\star}\right)_{k}^{i} \mathbb{P}_{i m}^{\star} \circ \lambda^{\star} d s,
\end{aligned}
$$

where Assumption $\left(\mathrm{A}_{2}\right)$ gives a meaning to $\widetilde{\mathbb{P}}_{i m}^{\star}$ on $L^{\star}$ (i.e., to $\widetilde{\mathbb{P}}_{i m}^{\star} \circ \lambda^{\star}$ on $S^{1}$ ) and hence to the duality pairing

$$
\delta W_{\mathrm{e}}^{\circ}\left(\lambda^{\star}\right)[\lambda]=\left\langle W_{F}^{\star}, D S\left(\lambda^{\star}\right)[\lambda]\right\rangle,
$$

completing the proof.

The integrand in the right-hand side of (4-9) is recognized as the Peach-Koehler force. Theorem 24 simply says that at minimality the virtual work done by the Peach-Koehler force must vanish.

Remark 25. The duality pairing (4-11) holds as soon as one considers a mollification of $W_{F}^{\star}$, that is, if $W_{F}^{\star}$ is assumed continuous. However, this assumption is stronger than $\left(\mathrm{A}_{2}\right)$, which requires only the continuity of some physically relevant components (related to the formation of a cavitation at the line singularity). Furthermore, nothing guarantees that the variation with any mollification of $W_{F}^{\star}$ would vanish since it is strictly speaking not the minimum point. Thus, at the mesoscopic scale, the best assumption found is $\left(\mathrm{A}_{2}\right)$ in order to be able to merely define the Peach-Koehler force as related to minimality. 
According to Remark 25, the following subsection shows how the Peach-Koehler force would formally be recovered.

Formal derivation of the Peach-Koehler force from the Eshelby tensor. Recalling (3-23), we introduce the Eshelby tensor $\mathscr{E}$ componentwise as

$$
\mathscr{E}_{i j}=\delta_{i j} W_{\mathrm{e}}-F_{k i} \mathbb{P}_{k j} .
$$

Then, assuming that $F$ and $\mathbb{P}$ are smooth enough,

$$
\partial_{j} \mathscr{E}_{i j}=\partial_{i} W_{\mathrm{e}}-\partial_{j} F_{k i} \mathbb{P}_{k j}-F_{k i} \partial_{j} \mathbb{P}_{k j}
$$

At minimality, one has $\partial_{j} \mathbb{P}_{k j}=0$ and hence

$$
\partial_{j} \mathscr{E}_{i j}^{\star}=\partial_{i} W_{\mathrm{e}}^{\star}-\partial_{j} F_{k i}^{\star} \mathbb{P}_{k j}^{\star}=\partial_{i} W_{\mathrm{e}}^{\star}-\left(\partial_{j} F_{k i}^{\star}-\partial_{i} F_{k j}^{\star}\right) \mathbb{P}_{k j}^{\star}-\partial_{i} F_{k j}^{\star}\left(W_{F}^{\star}\right)_{k j},
$$

where the first and last terms of the right-hand side mutually cancel, whence

$$
\partial_{j} \mathscr{E}_{i j}^{\star}=-\left(\partial_{j} F_{k i}^{\star}-\partial_{i} F_{k j}^{\star}\right) \mathbb{P}_{k j}^{\star}=\epsilon_{i j l} \epsilon_{l m n} \partial_{m} F_{k n}^{\star} \mathbb{P}_{k j}^{\star}=\epsilon_{i j l}\left(\operatorname{Curl} F^{\star}\right)_{k l} \mathbb{P}_{k j}^{\star},
$$

that is

$$
\partial_{j} \mathscr{E}_{i j}^{\star}=\left\langle\epsilon_{i l j} \Lambda_{l k}^{\star}, \mathbb{P}_{k j}^{\star}\right\rangle .
$$

Note that (4-16) has no rigorous meaning in our setting, i.e., at the mesoscale, since, $\Lambda^{\star}$ being a Radon measure but $\mathbb{P}^{\star}$ not being continuous, the duality pairing (4-16) is undefined. This is the reason why the Peach-Koehler force is established in our work by means of Assumption $\left(\mathrm{A}_{2}\right)$.

4.4. Configurational balance. Let $L=\gamma^{\star}\left(S^{1}\right)$ be a single smooth-enough dislocation loop with tangent vector $\tau$, normal vector $\nu$, curvature $\kappa$, and total Burgers vector $B$. This is assumed for simplicity of exposition, but similar results can be stated for general $\lambda^{\star} \in W^{1,1}\left(S^{1}, \widehat{\Omega}^{3}\right)$. We introduce

$$
\begin{aligned}
\mathscr{F} & :=\left(\mathbb{P}^{\star} \times \tau\right)^{\mathrm{T}} B \delta_{L}, \\
\mathscr{G} & :=\kappa(\psi(b, \tau)-\nabla \psi(b, \tau) \cdot \tau+\nabla \nabla \psi(b, \tau) \cdot \nu \otimes \nu) \nu\left\|\dot{\gamma}^{\star}\right\|^{-1} \delta_{L},
\end{aligned}
$$

the so-called Peach-Koehler force and line tension, respectively, where $\psi$ is the energy density as introduced in (3-25).

Deriving strong forms of equilibrium from a variational problem is classically done provided some regularity of the minimizers is assumed, as summarized in the following theorem. Note that restricting to a single generating loop with Burgers vector $b$ is chosen for simplicity of exposition. In order to well-define tangent and normal vectors, as well as line curvature, the following regularity assumption will be made on the optimal dislocation set $L^{\star}=\gamma^{\star}\left(S_{1}\right)$ :

$\left(\mathrm{A}_{3}\right) \gamma^{\star} \in W^{2,1}\left(S^{1}, \widehat{\Omega}\right)$. 
Theorem 26. Under the assumptions of Theorem 21 , assuming $\psi, \bar{\psi}: \mathbb{Z}^{3} \times \mathbb{R}^{3} \rightarrow$ $\mathbb{R}^{+}$are of class $\mathscr{C}^{2}$ and that the optimal dislocation network satisfies $\left(\mathrm{A}_{3}\right)$ and is associated to a single Burgers vector $b$, then minimality implies equilibrium of configurational forces, in the sense that the Peach-Koehler force $\mathscr{F}^{\star}$ is balanced by the line tension $\mathscr{G}^{\star}$ in $L^{\star}$, i.e.,

$$
\mathscr{F}^{\star}+\mathscr{G}^{\star}=0 .
$$

Proof. Let us particularize (4-10) to the case where the density $\Lambda^{\star}$ is generated by one single loop $\gamma^{\star} \in W^{1,1}\left(S^{1}, \widehat{\Omega}\right)$ with Burgers vector $b=\beta_{i} e_{i}, \beta_{i} \in \mathbb{Z}(b \neq 0)$ (see Remark 19). For variations of the form $\gamma^{\star}+\epsilon \gamma$ with $\gamma \in W^{1, \infty}\left(S^{1}, \widehat{\Omega}\right),(4-10)$ becomes

$$
\begin{aligned}
\delta W_{\mathrm{e}}^{\circ}\left(\gamma^{\star}\right)[\gamma] & =\int_{S^{1}} \epsilon_{k j m}\left(\mathbb{P}^{\star} \circ \gamma^{\star}(s)\right)_{i m} \tau_{k} b_{i} \gamma_{j}(s)\left\|\dot{\gamma}^{\star}(s)\right\| d s \\
& =\int_{L^{\star}} \epsilon_{k j m} \mathbb{P}_{i m}^{\star} \tau_{k} b_{i} \gamma_{j} d \mathcal{H}^{1}=\int_{\widehat{\Omega}} \epsilon_{k j m} \mathbb{P}_{i m}^{\star} \gamma_{j} d \Lambda_{k i}^{\star} .
\end{aligned}
$$

Using the notation introduced in (4-8), we write

$$
W^{\circ}\left(\gamma^{\star}+\epsilon \gamma\right)=W_{\mathrm{e}}^{\circ}\left(\gamma^{\star}+\epsilon \gamma\right)+W_{\text {defect }}^{\circ}\left(\gamma^{\star}+\epsilon \gamma\right),
$$

We have

$$
\delta W^{\circ}\left(\gamma^{\star}\right)[\gamma]=\delta W_{\mathrm{e}}^{\circ}\left(\gamma^{\star}\right)[\gamma]+\delta W_{\text {defect }}^{\circ}\left(\gamma^{\star}\right)[\gamma] .
$$

Let us now compute the variation of the defect part of the energy. For a dislocation density of the form $\mu=b \otimes \gamma_{\sharp} \llbracket\left[S^{1} \rrbracket\right.$, (3-25) can be written as

$$
W_{\text {defect }}^{1}(\mu)=\int_{S^{1}} \psi\left(b, \frac{\dot{\gamma}}{\|\dot{\gamma}\|}(s)\right)\|\dot{\gamma}(s)\| d s .
$$

Taking into account that the term $\mathcal{W}_{\text {defect }}^{2}(\mu)$ does not change for small perturbations of the dislocation line, the first variation of (4-21) at the point $\gamma^{\star} \in W^{1,1}\left(S^{1}, \widehat{\Omega}\right)$ can be explicitly computed and will coincide with $\delta W_{\text {defect }}^{o}\left(\gamma^{\star}\right)[\gamma]$. It holds that

$$
\begin{array}{r}
\delta W_{\text {defect }}^{\circ}\left(\gamma^{\star}\right)[\gamma]=\int_{S^{1}}\left(D_{k} \psi\left(b, \frac{\dot{\gamma}^{\star}}{\left\|\dot{\gamma}^{\star}\right\|}(s)\right)\left(\frac{\dot{\gamma}_{k}\left\|\dot{\gamma}^{\star}\right\|^{2}-\dot{\gamma}_{k}^{\star} \dot{\gamma}_{j}^{\star} \dot{\gamma}_{j}}{\left\|\dot{\gamma}^{\star}\right\|^{2}}(s)\right)\right. \\
\left.+\psi\left(b, \frac{\dot{\gamma}^{\star}}{\left\|\dot{\gamma}^{\star}\right\|}(s)\right)\left(\frac{\dot{\gamma}_{j}^{\star} \dot{\gamma}_{j}}{\left\|\dot{\gamma}^{\star}\right\|}(s)\right)\right) d s,
\end{array}
$$

where $D_{k} \psi$ is the derivative of $\psi$ with respect to the $k$-th component of its second variable. Denoting $\tau=\dot{\gamma}^{\star} /\left\|\dot{\gamma}^{\star}\right\|$, we integrate by parts to obtain

$$
\begin{aligned}
\delta \widehat{\mathscr{W}}_{\text {defect }}\left(\gamma^{\star}\right)[\gamma]=-\int_{S^{1}}\left(\psi(b, \tau) \dot{\tau}_{j}-D_{k} \psi(b, \tau) \tau_{k} \dot{\tau}_{j}\right. \\
\\
\left.+D_{j} D_{k} \psi(b, \tau) \dot{\tau}_{k}-D_{p} D_{k} \psi(b, \tau) \dot{\tau}_{k} \tau_{p} \tau_{j}\right) \gamma_{j} d s,
\end{aligned}
$$


where we dropped the variable $s$. Equivalently, recalling that $\dot{\tau}_{i}=\kappa v_{i}$ and since $D_{j} D_{k} \psi(b, \tau) \dot{\tau}_{k}=\tau_{j}^{i} \tau_{p}^{i} D_{p} D_{k} \psi(b, \tau) \dot{\tau}_{k}+v_{j} v_{p} D_{p} D_{k} \psi(b, \tau) \dot{\tau}_{k}$,

$$
\begin{aligned}
g_{j}^{\star}[b] & :=\psi(b, \tau) \dot{\tau}_{j}^{i}-D_{k} \psi(b, \tau) \tau_{k} \dot{\tau}_{j}^{i}+D_{j} D_{k} \psi(b, \tau) \dot{\tau}_{k}-D_{p} D_{k} \psi(b, \tau) \dot{\tau}_{k} \tau_{p}^{i} \tau_{j}^{i} \\
& =\psi(b, \tau) \dot{\tau}_{j}^{i}-D_{k} \psi(b, \tau) \tau_{k} \dot{\tau}_{j}^{i}+D_{p} D_{k} \psi(b, \tau) \dot{\tau}_{k} v_{p} v_{j} \\
& =\kappa\left(\psi(b, \tau)-D_{k} \psi(b, \tau) \tau_{k}+D_{p} D_{k} \psi(b, \tau) v_{p} v_{k}\right) v_{j} .
\end{aligned}
$$

Plugging the last expression into (4-20) and using (4-18), we obtain

$$
\delta W^{\circ}\left(\gamma^{\star}\right)[\gamma]=\int_{S^{1}}\left(\epsilon_{k j m}\left(\mathbb{P}^{\star} \circ \gamma^{\star}\right)_{i m}(s) b_{i} \dot{\gamma}_{k}^{\star}(s)-g_{j}^{\star}[b](s)\right) \gamma_{j}(s) d s .
$$

From the condition

$$
\delta W^{\circ}\left(\gamma^{\star}\right)[\gamma]=0 \quad \text { for all } \gamma \in W^{1,1}\left(S^{1}, \mathbb{R}^{3}\right),
$$

due to the minimality of $\gamma^{\star}$, we then get from $(4-24) \mathscr{F}_{j}^{\star}+\mathscr{G}_{j}^{\star}=0$, with

$$
\mathscr{F}_{j}^{\star}:=\epsilon_{k j m}\left(\mathbb{P}^{\star}\right)_{i m} B_{i} \tau_{k} \delta_{L^{\star}} \quad \text { and } \quad \mathscr{G}_{j}^{\star}:=\rho_{\text {dislo }}(B) v_{j} \delta_{L^{\star}},
$$

where

$$
\rho_{\text {dislo }}(B):=-g^{\star}[B] \varepsilon^{-1}
$$

with $g^{\star}:=g_{j}^{\star} v_{j}, \varepsilon=\varepsilon(P):=\left\|\dot{\gamma}^{\star} \circ \gamma^{\star^{-1}}(P)\right\|$, the local deformation of the curve at $P \in L^{\star}$,

$$
B:=\theta(P) b,
$$

the total Burgers vector, and $\theta(P)$ as defined by (3-10), the multiplicity of the dislocation (accounting for the loops of the cluster whose Burgers vector is a multiple of $b$ ).

Remark 27. Actually, (4-17) holds at $\mathscr{H}^{1}$-a.e. $P \in L$ and not at all $P$. This is due to the fact that it might happen that a point $P \in L$ is the overlapping of parts of $\gamma$ which, although having the same tangent vector $\tau$, do not have the same curvature $\kappa$ nor the same orthogonal vector $v$.

In the case where $\theta=1$ and the dislocation is parametrized by arc length $\left(\left|\dot{\gamma}^{\star}\right|=1\right)$, the balance of forces can be rewritten as

$$
\epsilon_{k j m} \mathbb{P}_{i m}^{\star} b_{i} \tau_{k}=g_{j}^{\star}[b] \quad \text { on } L^{\star} .
$$

A modeling example. Conti et al. [2015a] consider a potential $\mathscr{W}_{\text {dislo }}^{1}$ of the form (3-25) with

$$
\psi(b, \tau):=|b|^{2}+\eta\langle b, \tau\rangle^{2},
$$

where $\eta>0$ is a constant. 
In the particular case where $b=\beta e_{1}, \beta \geq 1$, it is shown that such energy is also lower-semicontinuous.

In such a case, the above computations entail that

$$
\mathscr{G}_{j}^{\star}(P)=\left(|b|^{2}-\eta\langle b, \tau\rangle^{2}+2 \eta\langle b, v\rangle^{2}\right) \kappa v_{j}
$$

so that at the minimum of the energy

$$
\theta^{2}\left((1-\eta)\langle b, \tau\rangle^{2}+(1+2 \eta)\langle b, v\rangle^{2}\right) \kappa v_{j}=\epsilon_{j p k} \mathbb{P}_{i p}^{\star} \theta b_{i} \tau_{k} .
$$

\subsection{Some additional remarks.}

Formal balance of configurational forces. Equation (4-18) yields, by (4-16) and a slight abuse of notation,

$$
\delta W_{\mathrm{e}}^{\circ}\left(\gamma^{\star}\right)[\gamma]=\int_{\widehat{\Omega}}-\partial_{k} \mathscr{E}_{j k}^{\star} \gamma_{j} d x
$$

Therefore, (4-24) and (4-27) can be rewritten as the virtual configurational work balance at minimality, i.e.,

$$
-\operatorname{Div} \mathscr{E}^{\star}=\mathscr{G}^{\star},
$$

where $\mathscr{E}^{\star}$ and $\mathscr{G}^{\star}$ stand for the configurational stress and the internal configurational force [Gurtin 2000, p. 34]. In our case, $\mathscr{G}^{\star}:=\rho_{\mathrm{dislo}}[B] v_{j} \delta_{L^{\star}}$. Quoting Gurtin, such force is "related to the material structure of the body $\mathscr{B}$; to each configuration of $\mathscr{B}$ there correspond a distribution of material and internal configurational forces that act to hold the material in place in that configuration. Such forces characterize the resistance of the material to structural changes and are basic when discussing temporal changes associated with phenomena such as the breaking of atomic bonds during fracture [and during dislocation motion]".

Let us note that Agiasofitou and Lazar [2010] have also derived a relation such as (4-28) in the framework of dislocation gauge theory by means of invariance properties and the Noether theorem (without considering a minimization problem as done here). These authors showed that the translational balance laws of the elastic and dislocation parts give rise to the Peach-Koehler force and also give the interpretation that "the Peach-Koehler force is the interaction force between the elastic subsystem and the dislocation subsystem" (see also (5.39) in [Lazar and Anastassiadis 2008]).

A brief glance at the dynamic problem. So far, we have identified the stationarity condition as a balance of configurational work. This happens when minimality is reached.

Consider now a time-evolution problem involving dislocation lines. In principle, no variational problem drives its evolution instantaneously, but minimality might be reached as $t \rightarrow \infty$ [Berdichevsky 2006]. So a first remark is that, before minimality 
is reached, one has Div $\mathscr{E}^{\star}+g^{\star} \neq 0$, by definition, and hence there exists a nonzero momentum $p$ such that, according to [Gurtin 2000, p. 46],

$$
-F^{\mathrm{T}} \dot{p}=\operatorname{Div} \mathscr{E}^{\star}+\mathscr{G}^{\star} .
$$

Hence, one might determine the motion of the line towards equilibrium, i.e., until $\dot{p}=0$.

Now, Gurtin [2000, p. 11] further says that as far as $\dot{p}=0$ the internal configurational force remains "indeterminate when and only when the associated structures are fixed in the material". This is similar to the constraint forces in classical mechanics (as the line tension of the pendulum) which do not need to be determined to establish the motion equation. In particular, no constitutive law for these forces is required in general.

However, we would like to emphasize that we have derived a constitutive law since (4-28) can be rewritten as

$$
\text { Peach-Koehler force }=\operatorname{Div} \mathscr{E}^{\star}=-\rho_{\text {dislo }}[B] v_{j} \delta_{L^{\star}},
$$

where $\rho_{\text {dislo }}$ is given in extenso by (4-23) and (4-25) in terms of the dislocation energy.

Second Euler-Lagrange equation and the dislocation equation. With the view of establishing an equation relating dislocation density and stress, the second EulerLagrange equation for our minimum problem should be derived; that is, the differential of the total energy should be computed with respect to divergence-free deformations $G$ (recall that curl-free deformations were considered for the first Euler-Lagrange equation in Section 4.2). We would like to point out serious mathematical issues in order to give a meaning to a vanishing of such a variation, $0=\delta \mathscr{W}\left(F^{\star}\right)[G]$. The principal reason (and the only which we discuss here) is that the differential $\frac{d}{d \epsilon} W_{\text {dislo }}\left(\Lambda^{\star}+\epsilon \Lambda\right)_{\mid \epsilon=0}$ has no meaning in $\mathcal{M}_{\Lambda}\left(\widehat{\Omega}, \mathbb{M}^{3}\right)$, this space not being linear, due to the fact that, as $\epsilon$ tends to zero, the resulting Burgers vector might not be an integer, whereas the minimum is achieved in this class of measures, with a crystallographic Burgers vector.

\section{Concluding remarks}

On the way to mathematically understanding time evolution of dislocations, the work achieved in [Scala and Van Goethem 2016] was the first step, allowing us to describe the geometry of dislocation clusters and to prove existence of solutions to a general variational problem. With the present contribution, our wish was to provide a further decisive step since the result of Theorem 26 introduces two forces balancing each other at optimality, the first deriving from the elastic part of the energy and named after Peach and Koehler (well-known in dislocation models [Hirth 
and Lothe 1982]) and the second deriving by shape variation of the defect part of the energy. Here crucial use has been made of the decomposition $F=\nabla u+F^{\circ}$ where $F^{\circ}$ and $\operatorname{Curl} F^{\circ}$ depend on the line. Such a force and such a balance of forces could be derived at the mesoscopic scale, without the required mathematical formalism, since there is subtle interplay between concentrated measures and Sobolev functions.

It turns out that the sum of these two forces naturally provides an expression of the velocity of the dislocation (for instance, a linear law is acceptable under certain working assumptions [Acharya 2003]). Of course, a nonvanishing velocity, i.e., a nonzero force, means that the solution does not coincide with energy minimization, as is well-known for evolution problems. In future work, our task is to determine the dissipative effects and the balance equations and to analyze in detail the evolutionary scheme.

The force we derived here yields an important output in terms of modeling, but to achieve a proof of Theorem 26, a series of results have appeared about the mathematical nature of functional spaces for dislocation-induced deformations. These should also be considered as contributions to the general aim of understanding dislocation problems considered at the mesoscale in appropriate mathematical terms. Moreover, the paper has been written with a first part containing generic results, which are not related to dislocation models.

\section{References}

[Acharya 2003] A. Acharya, "Driving forces and boundary conditions in continuum dislocation mechanics”, R. Soc. Lond. Proc. Ser. A Math. Phys. Eng. Sci. 459:2034 (2003), 1343-1363.

[Agiasofitou and Lazar 2010] E. Agiasofitou and M. Lazar, "On the nonlinear continuum theory of dislocations: a gauge field theoretical approach”, J. Elasticity 99:2 (2010), 163-178.

[Ambrosio et al. 2000] L. Ambrosio, N. Fusco, and D. Pallara, Functions of bounded variation and free discontinuity problems, Clarendon, New York, 2000.

[Ball 2002] J. M. Ball, "Some open problems in elasticity", pp. 3-59 in Geometry, mechanics, and dynamics, edited by P. Newton et al., Springer, New York, 2002.

[Berdichevsky 2006] V. L. Berdichevsky, "Continuum theory of dislocations revisited", Contin. Mech. Thermodyn. 18:3-4 (2006), 195-222.

[Berezhkova 1969] G. V. Berezhkova, Nitevidnye kristally, Nauka, Moscow, 1969.

[Bolik and von Wahl 1997] J. Bolik and W. von Wahl, "Estimating $\nabla \boldsymbol{u}$ in terms of div $\boldsymbol{u}, \operatorname{curl} \boldsymbol{u}$, either $(v, \boldsymbol{u})$ or $v \times \boldsymbol{u}$ and the topology", Math. Methods Appl. Sci. 20:9 (1997), 737-744.

[Bourgain and Brezis 2004] J. Bourgain and H. Brezis, "New estimates for the Laplacian, the divcurl, and related Hodge systems", C. R. Math. Acad. Sci. Paris 338:7 (2004), 539-543.

[Cermelli and Gurtin 2001] P. Cermelli and M. E. Gurtin, "On the characterization of geometrically necessary dislocations in finite plasticity”, J. Mech. Phys. Solids 49:7 (2001), 1539-1568.

[Conti et al. 2011] S. Conti, A. Garroni, and S. Müller, "Singular kernels, multiscale decomposition of microstructure, and dislocation models", Arch. Ration. Mech. Anal. 199:3 (2011), 779-819. 
[Conti et al. 2015a] S. Conti, A. Garroni, and A. Massaccesi, "Modeling of dislocations and relaxation of functionals on 1-currents with discrete multiplicity", Calc. Var. Partial Differential Equations 54:2 (2015), 1847-1874.

[Conti et al. 2015b] S. Conti, A. Garroni, and M. Ortiz, "The line-tension approximation as the dilute limit of linear-elastic dislocations", Arch. Ration. Mech. Anal. 218:2 (2015), 699-755.

[Cottrell 1964] A. H. Cottrell, Theory of crystal dislocations, Gordon and Breach, New York, 1964.

[Fabes et al. 1998] E. Fabes, O. Mendez, and M. Mitrea, "Boundary layers on Sobolev-Besov spaces and Poisson's equation for the Laplacian in Lipschitz domains", J. Funct. Anal. 159:2 (1998), 323368.

[Federer 1969] H. Federer, Geometric measure theory, Die Grundlehren der mathematischen Wissenschaften 153, Springer, New York, 1969.

[Fosdick and Royer-Carfagni 2004] R. Fosdick and G. Royer-Carfagni, "Stress as a constraint reaction in rigid bodies", J. Elasticity 74:3 (2004), 265-276.

[Fosdick and Royer-Carfagni 2005] R. Fosdick and G. Royer-Carfagni, "A penalty interpretation for the Lagrange multiplier fields in incompressible multipolar elasticity theory", Math. Mech. Solids 10:4 (2005), 389-413.

[Fujiwara and Morimoto 1977] D. Fujiwara and H. Morimoto, "An $L_{r}$-theorem of the Helmholtz decomposition of vector fields", J. Fac. Sci. Univ. Tokyo Sect. IA Math. $24: 3$ (1977), 685-700.

[Galdi 2011] G. P. Galdi, An introduction to the mathematical theory of the Navier-Stokes equations: steady-state problems, 2nd ed., Springer, New York, 2011.

[Giaquinta et al. 1998] M. Giaquinta, G. Modica, and J. Souček, Cartesian currents in the calculus of variations, II: Variational integrals, Ergebnisse der Mathematik und ihrer Grenzgebiete (3) 38, Springer, Berlin, 1998.

[Gurtin 2000] M. E. Gurtin, Configurational forces as basic concepts of continuum physics, Applied Mathematical Sciences 137, Springer, New York, 2000.

[Hirth and Lothe 1982] J. P. Hirth and J. Lothe, Theory of dislocations, 2nd ed., Wiley, New York, 1982.

[Hochrainer 2013] T. Hochrainer, "Moving dislocations in finite plasticity: a topological approach", Z. Angew. Math. Mech. 93:4 (2013), 252-268.

[Hochrainer and Zaiser 2005] T. Hochrainer and M. Zaiser, "Fundamentals of a continuum theory of dislocations", in International Conference on Statistical Mechanics of Plasticity and Related Instabilities (Bangalore, India, 2005), edited by S. Dattagupta et al., Proceedings of Science, Trieste, Italy, 2005.

[Kozono and Yanagisawa 2009] H. Kozono and T. Yanagisawa, " $L^{r}$-variational inequality for vector fields and the Helmholtz-Weyl decomposition in bounded domains", Indiana Univ. Math. J. 58:4 (2009), 1853-1920.

[Krantz and Parks 2008] S. G. Krantz and H. R. Parks, Geometric integration theory, Birkhäuser, Boston, 2008.

[Kröner 1981] E. Kröner, "Continuum theory of defects", pp. 219-315 in Physiques des défauts, session XXXV (Les Houches, France, 1980), edited by R. Balian et al., North-Holland, Dordrecht, 1981.

[Lazar and Anastassiadis 2008] M. Lazar and C. Anastassiadis, "The gauge theory of dislocations: conservation and balance laws”, Philos. Mag. 88:11 (2008), 1673-1699.

[Müller and Palombaro 2008] S. Müller and M. Palombaro, "Existence of minimizers for a polyconvex energy in a crystal with dislocations", Calc. Var. Partial Differential Equations 31:4 (2008), $473-482$. 
[Müller et al. 2004] G. Müller, J.-J. Métois, and P. Rudolph (editors), Crystal growth: from fundamentals to technology, Elsevier, Amsterdam, 2004.

[Neff et al. 2012a] P. Neff, D. Pauly, and K.-J. Witsch, "Maxwell meets Korn: a new coercive inequality for tensor fields in $\mathbb{R}^{N \times N}$ with square-integrable exterior derivative", Math. Methods Appl. Sci. 35:1 (2012), 65-71.

[Neff et al. 2012b] P. Neff, D. Pauly, and K.-J. Witsch, "On a canonical extension of Korn's first and Poincaré's inequality to H(Curl)", J. Math. Sci. 185:5 (2012), 721-727.

[Neff et al. 2015a] P. Neff, I. D. Ghiba, M. Lazar, and A. Madeo, "The relaxed linear micromorphic continuum: well-posedness of the static problem and relations to the gauge theory of dislocations", Quart. J. Mech. Appl. Math. 68:1 (2015), 53-84.

[Neff et al. 2015b] P. Neff, D. Pauly, and K.-J. Witsch, "Poincaré meets Korn via Maxwell: extending Korn's first inequality to incompatible tensor fields", J. Differential Equations 258:4 (2015), $1267-1302$.

[Scala and Van Goethem 2015] R. Scala and N. Van Goethem, "Graphs of torus-valued harmonic maps, with application to a variational model for dislocations", preprint, 2015, Available at https:// hal.archives-ouvertes.fr/hal-01183365v1.

[Scala and Van Goethem 2016] R. Scala and N. Van Goethem, "Currents and dislocations at the continuum scale", Methods Appl. Anal. 23:1 (2016), 1-34.

[Simader and Sohr 1996] C. G. Simader and H. Sohr, The Dirichlet problem for the Laplacian in bounded and unbounded domains: a new approach to weak, strong and $(2+k)$-solutions in Sobolevtype spaces, Pitman Research Notes in Mathematics Series 360, Longman, Harlow, England, 1996.

[Van Goethem 2014] N. Van Goethem, "Thermodynamic forces in single crystals with dislocations", Z. Angew. Math. Phys. 65:3 (2014), 549-586.

[Van Goethem 2015] N. Van Goethem, "Cauchy elasticity with dislocations in the small strain assumption”, Appl. Math. Lett. 46 (2015), 94-99.

[Van Goethem and Dupret 2012a] N. Van Goethem and F. Dupret, "A distributional approach to 2D Volterra dislocations at the continuum scale", European J. Appl. Math. 23:3 (2012), 417-439.

[Van Goethem and Dupret 2012b] N. Van Goethem and F. Dupret, "A distributional approach to the geometry of $2 D$ dislocations at the continuum scale", Ann. Univ. Ferrara Sez. VII Sci. Mat. 58:2 (2012), 407-434.

[von Wahl 1992] W. von Wahl, "Estimating $\nabla u$ by $\operatorname{div} u$ and curl $u$ ", Math. Methods Appl. Sci. 15:2 (1992), 123-143.

[Yanagisawa 2007] T. Yanagisawa, "Hodge decomposition of $L^{r}$-vector fields on a bounded domain and its application to the Navier-Stokes equations", RIMS Kokyuroku 1536 (2007), 73-86.

[Zaiser 2004] M. Zaiser, "Dislocation patterns in crystalline solids: phenomenology and modelling", pp. 215-238 in Crystal growth: from fundamentals to technology, edited by G. Müller et al., Elsevier, Amsterdam, 2004.

[Zubov 1997] L. M. Zubov, Nonlinear theory of dislocations and disclinations in elastic bodies, Lecture Notes in Physics Monographs 47, Springer, Berlin, 1997.

Received 8 Oct 2015. Accepted 17 Jul 2016.

RICCARDO SCALA: rscala@fc.ul.pt

Departamento de Matemática, Centro de Matemática, Aplicações Fundamentais e Investigação Operacional, Universidade de Lisboa, Alameda da Universidade C6, 1749-016 Lisboa, Portugal

Nicolas VAn GoETHEM: vangoeth@fc.ul.pt 
Departamento de Matemática, Centro de Matemática, Aplicações Fundamentais e Investigação Operacional, Universidade de Lisboa, Alameda da Universidade C6, 1749-016 Lisboa, Portugal 


\title{
STABILITY ANALYSIS OF TWO COUPLED OSCILLATORS
}

\author{
Mickhail A. Guzev And Alexandr A. DMitriev
}

\begin{abstract}
We study a system of two coupled oscillators linked by a linear elastic spring and positioned vertically in a uniform gravity field. It is demonstrated that the system has different equilibrium configurations below and above the oscillators' suspension centers. We obtained the relations of the string stiffness and the distance between the suspension centers identifying the stability region of the oscillators.
\end{abstract}

\section{Introduction}

Mechanical oscillators are models of various physical processes and complex physical systems as demonstrated by a vast body of literature. For example, coupled oscillators are used to describe the lattice vibrations in crystals [Kittel 2005].

A well-known and useful oscillator system is the sympathetic oscillators [Sommerfeld 1994], which are two linked oscillators with equal rods and masses interacting through a spring. Small linear oscillations about the equilibrium point have been studied, focusing on analyzing the physical situations depending on the spring stiffness.

There have been many scientific studies on oscillating dynamics of mechanical systems. However, new results still periodically appear. For instance, Maianti et al. [2009] study the impact of symmetrical initial conditions of linked oscillators in a uniform gravity field on the eigenoscillations and obtain the initial angle that ensures an independent frequency spectrum. Ramachandran et al. [2011] deal with different configurations of two pendulums connected by a rod. The results are that there are stable equilibrium configurations that are asymmetrical with respect to the vertical midline. An important property of the system is that there can appear bifurcations depending on the distance between the suspension points. The obtained results are useful for investigation of the pantographic structures [dell' Isola et al. 2016]. The interest in these materials is defined by development of the threedimensional printing technology. They can be regarded as families of pendulums (also called fibers) interconnected by pivots in equilibrium. Synchronization of

\section{Communicated by Francesco dell'Isola.}

MSC2010: 70E55, 70H14.

Keywords: coupled oscillators, equilibrium configurations, stability, linear interaction. 
two oscillators is the focus of [Koluda et al. 2014] and their chaotic dynamics is studied in [Huynh and Chew 2010; Huynh et al. 2013].

A system of inverted oscillators also provides physically sound phenomena. Stable positions can also be attained if there is a fast perturbation frequency [Stephenson 1908]. This result is due to Pyotr Kapitza [Kapitza 1951a; Kapitza 1951b]. A more accurate condition of dynamical stabilization of an inverted oscillator is introduced in [Butikov 2011]. Chelomei's problem of the stabilization of an elastic, statically unstable rod by means of a vibration is considered in [Seyranian and Seyranian 2008]. The stability of two inverted linearly linked oscillators is analyzed in [Markeev 2013]. The author reveals bifurcations depending on the linking spring stiffness and single out parameters that lead to stable or unstable equilibria. The phenomenon of stabilization by parametric excitation of an elastically restrained double inverted pendulum is considered in [Arkhipova et al. 2012]. The problem of restabilization of statically unstable linear Hamiltonian systems is analyzed in [Arkhipova and Luongo 2014]. A comprehensive review of the dynamics of a large number of coupled oscillators is presented in [Pikovsky and Rosenblum 2015].

The objective of the current paper is to study the stability of the model of two linearly interacting oscillators in a uniform gravity field. The formal analysis of equilibrium stability is carried out in the framework of the linear stability approach. It consists of determination of the equilibrium position and calculation of the matrix of the second partial derivatives of potential energy in the equilibrium position. If the matrix spectrum is positive, the equilibrium is stable. Otherwise, it is unstable. We focus on analyzing the equilibrium solutions depending on the distance between the suspension points and the spring stiffness. This analysis includes different configurations of the model of coupled oscillators.

\section{Basic equations}

Let us consider two oscillators of length $l$ and mass $m$ in a uniform gravity field. We assume that the suspension points $O_{1}$ and $O_{2}$ are positioned on a motionless horizontal straight line, while the distance between the suspension points $a$ is constant. A massless elastic spring of stiffness $k$ links the masses at points $B_{1}$ and $B_{2}$, which coincide with the masses' positions. We assume that the oscillators move in a fixed vertical plane containing the interval $\mathrm{O}_{1} \mathrm{O}_{2}$ (see Figure 1). The oscillators can be situated both below the horizontal suspension line (see the region $A 1$ in Figure 1, left) and above it (see the region $A 2$ in Figure 1, right). In the region $A 1$, angles $\varphi_{1}$ and $\varphi_{2}$ lie in the interval $(0, \pi)$, while transition to the region $A 2$ implies the transformation $\varphi_{1}, \varphi_{2} \mapsto-\varphi_{1},-\varphi_{2}$. 

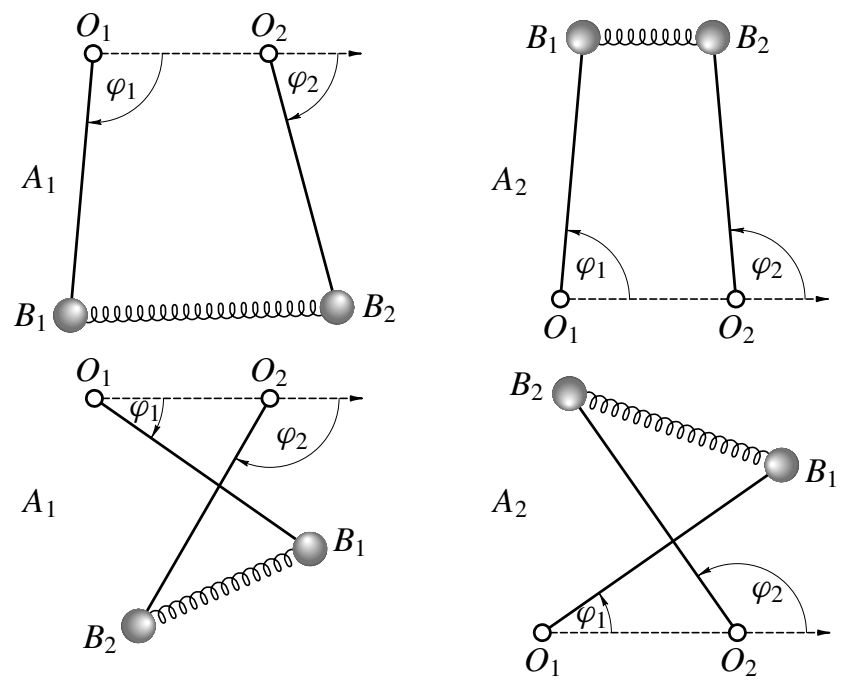

Figure 1. Top left: the classical configuration and the region $A 1$. Top right: the classical configuration and the region $A 2$. Bottom left: the modified configuration and the region $A 1$. Bottom right: the modified configuration and the region $A 2$.

Hence, in this article, we consider different configurations of the oscillator model. Configurations presented in Figure 1, top left, correspond to the sympathetic oscillators [Sommerfeld 1994], and configurations of Figure 1, top right, describe a system of inverted oscillators. Both models are well-known in scientific literature, so configurations presented in Figure 1, top, will be called the classical ones.

Configurations of Figure 1, bottom, are presented in [Ramachandran et al. 2011] (called "modified configurations" to distinguish them from Figure 1, top).

It is clear that the kinetic energy of the oscillators is

$$
T=\frac{m l^{2}}{2}\left[\left(\dot{\varphi}_{1}\right)^{2}+\left(\dot{\varphi}_{2}\right)^{2}\right]
$$

Potential energy $U$ includes the energy of the oscillator interaction $k(d-a)^{2} / 2$ and the gravity field energy where $d$ is the spring length. In the region $A 1$, oscillators linked by a linear elastic spring provide

$$
U=U\left(\varphi_{1}, \varphi_{2}\right)=\frac{k(d-a)^{2}}{2}-m g l\left(\sin \varphi_{1}+\sin \varphi_{2}\right)
$$

while in the region $A 2$ there is a transformation $g \mapsto-g$ in (2). In the regions $A 1$ and $A 2$, the spring length is given by the formula

$$
d=\sqrt{\left[a+l\left(\cos \varphi_{2}-\cos \varphi_{1}\right)\right]^{2}+l^{2}\left(\sin \varphi_{2}-\sin \varphi_{1}\right)^{2}} .
$$


It is interesting that there is a natural geometrical condition for the configurations. In the case of the classical configurations (Figure 1, top), the difference of the rod length projections on the suspension axis is less then $a$, giving the condition

$$
l\left(\cos \varphi_{1}-\cos \varphi_{2}\right)<a .
$$

In the case of the modified configurations (Figure 1, bottom), the corresponding difference is larger than $a$ :

$$
l\left(\cos \varphi_{1}-\cos \varphi_{2}\right)>a .
$$

From (1) and (2), the Lagrangian of the system ensures

$$
L=T-U=\frac{m l^{2}}{2}\left(\dot{\varphi}_{1}^{2}+\dot{\varphi}_{2}^{2}\right)-\frac{k(d-a)^{2}}{2}+2 m g l \sin \frac{\varphi_{1}+\varphi_{2}}{2} \cos \frac{\varphi_{1}-\varphi_{2}}{2} .
$$

Now let us introduce instead of $\varphi_{1}$ and $\varphi_{2}$ new coordinates $q_{1}$ and $q_{2}$, where $q_{1}=\left(\pi-\varphi_{1}-\varphi_{2}\right) / 2$ and $q_{2}=\left(\varphi_{1}-\varphi_{2}\right) / 2$. Introducing new dimensionless time $\tau=t \sqrt{2 g / l}$ and Lagrangian $\Lambda=L / m g l$, (5) can be rewritten as

$$
\begin{aligned}
& \Lambda=\frac{1}{2}\left(\dot{q}_{1}^{2}+\dot{q}_{2}^{2}\right)-\Pi\left(q_{1}, q_{2}\right), \\
& \Pi=\Pi\left(q_{1}, q_{2}\right)=\frac{(s-\mu)^{2}}{2 v}-\cos q_{1} \cos q_{2}, \\
& s^{2}=\sin ^{2} q_{2}+2 \mu \cos q_{1} \sin q_{2}+\mu^{2}, \quad \mu=\frac{a}{2 l}, \quad v=\frac{2 m g l}{k} .
\end{aligned}
$$

Parameter $v$ characterizes the relation between the potential energy of the oscillators and the spring's effective energy, while $\mu$ is a kinematic parameter and depends on the metric characteristics.

Differential equations of the oscillator dynamics in the form of Lagrangian equations are

$$
\frac{d}{d \tau} \frac{\partial \Lambda}{\partial \dot{q}_{i}}=\frac{\partial \Lambda}{\partial q_{i}} \quad \Longleftrightarrow \quad \ddot{q}_{i}=-\frac{\partial \Pi}{\partial q_{i}}, \quad i=1,2 .
$$

System (7) allows for solutions corresponding to both the classical and the modified configurations. Therefore, while analyzing system (7), it is necessary to point out the region of feasible solutions. Conditions (3)-(4) can be written as

$$
\begin{aligned}
& \mu+\cos q_{1} \sin q_{2}>0, \\
& \mu+\cos q_{1} \sin q_{2}<0 .
\end{aligned}
$$

Equilibrium configurations of the oscillator system ensue from the condition $\ddot{q}_{i}=0$; then it follows from (7) that they are determined as the critical points of the system's potential energy

$$
\frac{\partial \Pi}{\partial q_{1}}=0, \quad \frac{\partial \Pi}{\partial q_{2}}=0 .
$$



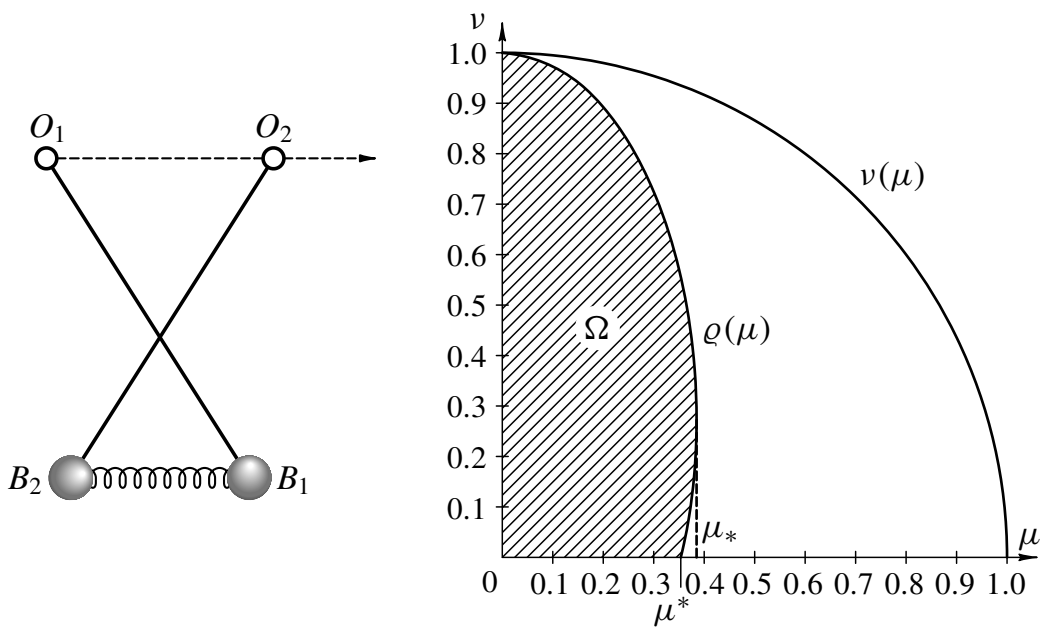

Figure 2. Left: the modified symmetric equilibrium configuration for the oscillator model in the region $A 1$. Right: the stability domain $\Omega$ for the modified configuration in the region $A 1$.

Taking into account (6), one can rewrite (10) in the form

$$
\begin{aligned}
\sin q_{1}\left[\left(\frac{\mu}{s}-1\right) \mu \sin q_{2}+v \cos q_{2}\right] & =0, \\
\left(1-\frac{\mu}{s}\right)\left(\sin q_{2}+\mu \cos q_{1}\right) \cos q_{2}+v \cos q_{1} \sin q_{2} & =0 .
\end{aligned}
$$

Thus, by solving the system (11)-(12), one obtains a set of equilibrium configurations.

\section{Symmetrical equilibrium configurations}

Symmetrical configurations are characterized by symmetrical positions of the pendulums with respect to the vertical midline. The classical symmetric configurations in the region $A 1$ follow from $q_{1}=0$, while in the region $A 2$ from $q_{1}=\pi$. In this case, (11) is satisfied identically ( $\sin q_{1}=0$ ); then the distance (6) between the oscillators equals $s=\left|\sin q_{2} \pm \mu\right|$ and the condition (8) is equivalent to $\mu \pm \sin q_{2}>0$, i.e., $s=\mu \pm \sin q_{2}$. So (12) reduces to $\sin q_{2}\left(\cos q_{2} \pm v\right)=0$, which was studied in [Markeev 2013].

The modified symmetrical configurations in the region $A 1$ follow from $\varphi_{2}=$ $\pi-\varphi_{1}, q_{1}=0$, and are shown in Figure 2. This allows us to rewrite the condition (9) as $\mu+\sin q_{2}<0$, i.e., $\mu<1$ and $\left|q_{2}\right|<\pi / 2$; then the distance $s=-\left(\mu+\sin q_{2}\right)$ and (12) is equivalent to

$$
\left(2 \mu+\sin q_{2}\right) \cos q_{2}+v \sin q_{2}=0
$$

$$
\Longleftrightarrow \quad \sin 2 q_{2}+2 \sqrt{4 \mu^{2}+v^{2}} \sin \left(q_{2}-q^{*}\right)=0,
$$


where $q^{*}=-\arcsin \left(2 \mu / \sqrt{4 \mu^{2}+v^{2}}\right)$. Let $q^{* *}=-\arcsin \mu$; then inside the interval $\left(q^{*}, q^{* *}\right),(13)$ has a unique solution $\tilde{q}$ provided the inequality

$$
v<\sqrt{1-\mu^{2}}, \quad \mu<1,
$$

is true. Indeed, (13) is identical to

$$
2 \mu+\sin q_{2}=-v \tan q_{2} .
$$

The right-hand side of (15) decreases; it equals $2 \mu / \nu$ at point $q^{*}$ and $\mu / \sqrt{1-\mu^{2}}$ at point $q^{* *}$. The left-hand side increases; it is less than $2 \mu / v$ at point $q^{*}$ and equals $2 \mu / v$ at point $q^{* *}$. If the inequality (14) is satisfied, the function graphs intersect at one and only one point $\tilde{q}$.

Let us analyze the type of equilibrium. The matrix of the second partial derivatives of potential $\Pi$ at critical point $(0, \tilde{q})$ agrees with

$$
\begin{aligned}
& \Pi_{11}=\frac{\partial^{2} \Pi}{\partial q_{1}^{2}}=\left(\frac{\mu}{s}-1\right) \frac{\mu}{v} \sin \tilde{q}+\cos \tilde{q}, \\
& \Pi_{22}=\frac{\partial^{2} \Pi}{\partial q_{2}^{2}}=\frac{1}{v}\left[\cos ^{2} \tilde{q}+\left(\frac{\mu}{s}-1\right)(\sin \tilde{q}+\mu) \sin \tilde{q}\right]+\cos \tilde{q}, \\
& \Pi_{12}=\frac{\partial^{2} \Pi}{\partial q_{1} \partial q_{2}}=0
\end{aligned}
$$

i.e., the matrix is diagonal. At point $\tilde{q}$, since $s=-(\mu+\sin \tilde{q})$, (13) is equivalent to $(s-\mu)=v \tan \tilde{q}$, which results in

$$
\Pi_{11}=\frac{\mu+\cos ^{2} \tilde{q} \sin \tilde{q}}{\cos \tilde{q}(\mu+\sin \tilde{q})}, \quad \Pi_{22}=\frac{1}{v} \cos ^{2} \tilde{q}+\frac{1}{\cos \tilde{q}} .
$$

It is straightforward that $\Pi_{22}>0$ and $\Pi_{11}>0$ if

$$
\mu+\cos ^{2} \tilde{q} \sin \tilde{q}<0 .
$$

To solve (17), one needs to find the roots of the cubic parabola $x^{3}-x-\mu$ as $x=\sin \tilde{q}$. It ensures the restrictions on parameter $\mu$

$$
0<\mu<\mu_{*}=\frac{2}{3 \sqrt{3}}, \quad x_{1}(\mu)<\sin \tilde{q}<x_{2}(\mu),
$$

where $x_{1}(\mu)$ and $x_{2}(\mu)$ are the cubic parabola's roots:

$$
\begin{aligned}
& x_{1}(\mu)=-\frac{2}{\sqrt{3}} \sin \left(\frac{\pi}{6}+\phi(\mu)\right), \\
& x_{2}(\mu)=-\frac{2}{\sqrt{3}} \sin \left(\frac{\pi}{6}-\phi(\mu)\right),
\end{aligned}
$$



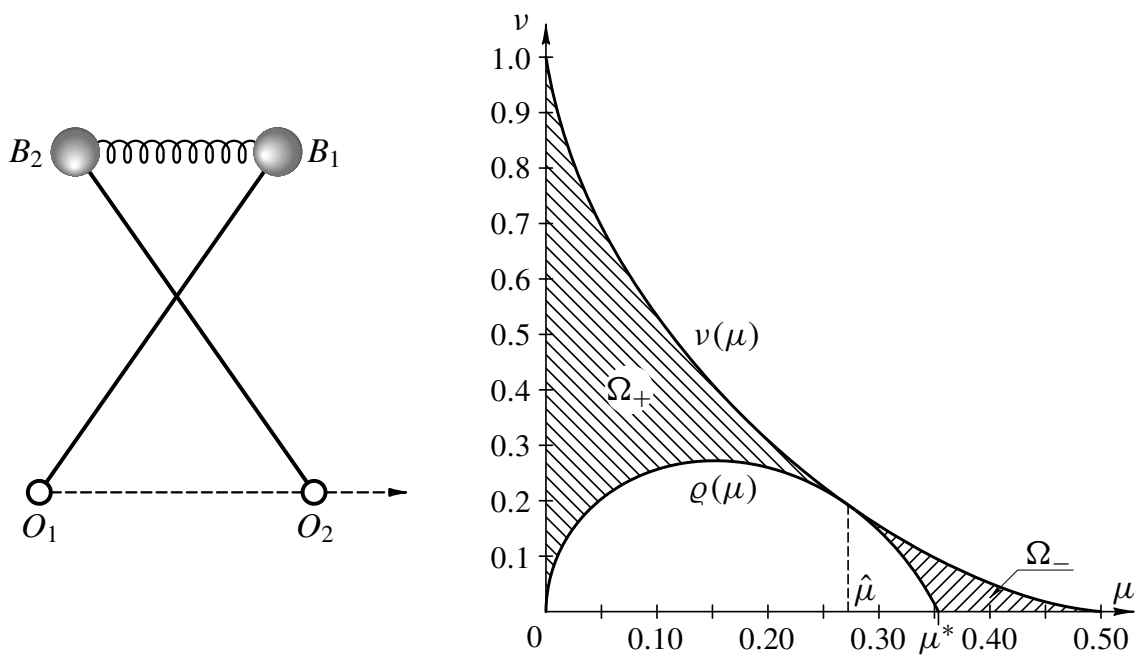

Figure 3. Left: the modified symmetric equilibrium configuration in the region $A 2$. Right: the solution existence domain for the modified symmetric equilibrium configuration in the region $A 2$. The region $\Omega_{-}$is the stability region.

Thus, the oscillator model in the region A1 given the condition (14) has modified equilibrium configurations depending on the solution $\tilde{q}$ of (13). This equilibrium is stable if the conditions (18) and (19) are satisfied.

Figure 2 shows that the region of solution existence is bounded by a circular $\operatorname{arc} \nu(\mu)=\sqrt{1-\mu^{2}}$. The shaded region $\Omega$ indicates parameters $(\mu, v)$ that ensure stable configuration. The boundary of the stability region $\varrho(\mu)$ is determined by $\Pi_{11}=0$. However, this formula is rather cumbersome; thus, it is not presented. It should be noted that $\varrho(\mu)$ has two branches merging at point $\mu_{*}$.

If a point $(\mu, v)$ is outside the domain $\Omega$, then the critical point corresponding to the solution $\tilde{q}$ of (13) is a saddle.

For the modified oscillator model, the equilibrium configurations in the region $A 2$ follow from $q_{1}=\pi\left(\varphi_{1}+\varphi_{2}=-\pi\right)$, the distance $s=\sin q_{2}-\mu>0$, i.e., $q_{2}>0$, and (12) takes the form

$$
\sin q_{2}=2 \mu+v \tan q_{2} .
$$

The oscillator position corresponding to the region $A 2$ is depicted in Figure 3.

Since $\sin q$ is a concave function as $q \in(0, \pi / 2)$ and $\tan q$ is convex, the number of solutions of (20) depends on the parameters $(\mu, v)$. Particularly, $q_{0}$ exists if the function graphs have a common tangent, i.e., $\cos q_{0}=v / \cos ^{2} q_{0}$. Substituting the obtained $v$ into (20), we get $2 \mu=\sin ^{3} q_{0}$. It follows that there is a curve

$$
v(\mu)=\left[1-(2 \mu)^{2 / 3}\right]^{3 / 2},
$$


whose points determine the only solution $q_{0}(\mu)=\arcsin (2 \mu)^{1 / 3}$ of (20). The solution $q_{0}(\mu)$ is a bifurcation point. If one slightly varies the parameters $(\mu, v)$, (20) has either no solution or two solutions $q_{-}$and $q_{+}\left(q_{-}<q_{0}(\mu)<q_{+}\right)$. From convexity of $\tan q$, concavity of $\sin q$, and (21), it follows that the condition for two solutions is

$$
v<\left[1-(2 \mu)^{2 / 3}\right]^{3 / 2}
$$

which leads to $\mu<\frac{1}{2}$.

By analogy to (16), one can infer that

$$
\Pi_{11}=\frac{\mu-\cos ^{2} q_{ \pm} \sin q_{ \pm}}{\cos q_{ \pm}\left(\mu-\sin q_{ \pm}\right)}, \quad \Pi_{22}=\cos ^{2} q_{ \pm}-\frac{v}{\cos q_{ \pm}} .
$$

The function $1-v / \cos ^{3} q$ decreases and equals zero at $q_{0}(\mu)$; therefore, $\Pi_{22}<0$ at the root $q_{+}$of (20). Hence, the oscillators are unstable around the equilibrium from $q_{+}$.

The value of $\Pi_{11}$ is positive in the region where $h(q)=\mu-\cos ^{2} q \sin q$ is positive. This region ensures that

$$
\sin q<x_{1}(\mu), \quad x_{2}(\mu)<\sin q, \quad 0<\mu<\mu_{*} .
$$

Figure 3 shows a shaded region $\Omega_{+}$, where $\Pi_{11}<0$ at $q_{+}$, and another shaded region $\Omega_{-}$, where $\Pi_{11}>0$ at $q_{-}$. The point $\hat{\mu}$ is a tangential point of curves $v(\mu)$ and $\varrho(\mu)$. Calculated values of $\hat{\mu} \approx 0.272166$ and $\hat{v} \approx 0.19245$.

Thus, in the region A2, the equilibria of the modified configurations are determined by the two solutions $q_{-}$and $q_{+}$of (20), which exist as the parameters $(\mu, v)$ comply with (21).

If the parameters $(\mu, v)$ are inside the region $\Omega_{+}$, the critical point corresponding to $q_{+}$is a maximum, while otherwise it is a saddle.

If the parameters $(\mu, v)$ are inside the region $\Omega_{-}$, the critical point corresponding to $q_{-}$is stable, while otherwise it is again a saddle.

\section{Asymmetric equilibrium configurations}

To study the asymmetric equilibria, it is convenient to use the variables $x=\sin q_{2}$ and $y=\cos q_{1}$. Since $-\pi / 2<q_{2}<\pi / 2$ and $0<q_{1}<\pi / 2$ in the region $A 1$ and $-\pi / 2<q_{1}<0$ in the region $A 2$, these transformations result in a one-to-one mapping in each of the considered regions. It is straightforward that the variables $x$ and $y$ vary within the triangle $\Delta_{+}=\{(x, y):-1<x<1,0<y<1\}$ in the region $A 1$ and $\Delta_{-}=\{(x, y):-1<x<1,-1<y<0\}$ in the region $A 2$. Using the variables $x$ and $y$, the potential $\Pi$ is given by

$$
\Pi(x, y)=\frac{(s-\mu)^{2}}{2 v} \mp \sqrt{1-x^{2}} \cdot y, \quad s^{2}=x^{2}+2 \mu x y+\mu^{2},
$$


where the minus corresponds to the region $A 1$ and the plus corresponds to $A 2$. Then the system (10) can be rewritten as

$$
\begin{aligned}
\mu \frac{s-\mu}{s} k(x) \mp \nu & =0, \quad k(x)=\frac{x}{\sqrt{1-x^{2}}} . \\
\frac{s-\mu}{s}(x+\mu y) \pm v k(x) y & =0,
\end{aligned}
$$

By eliminating $(s-\mu) / s$, we obtain the relation $\mu y+x\left(1-x^{2}\right)=0$, which suggests that the critical points of the potential $\Pi$ are determined from the system

$$
\begin{aligned}
h(x, \mu)=\mu \frac{s-\mu}{s} k(x) & = \pm v, \\
\mu y+x\left(1-x^{2}\right) & =0 .
\end{aligned}
$$

The left-hand side of (23) differs from the cubic parabola pertaining to (17), by a multiplicator $y$ at $\mu$.

Substituting (23) in the $s$ relation, one obtains

$$
s^{2}=2 x^{4}-x^{2}+\mu^{2}
$$

The triangle $\Delta_{+}$intersects the cubic parabola of (23) if

$$
\begin{aligned}
& -\sqrt{1-\mu} \leq x \leq x_{1}(\mu), \\
& x_{2}(\mu) \leq x \leq 0 \\
& \text { as } 0<\mu<\mu_{*} \text {, } \\
& -\sqrt{1-\mu} \leq x \leq 0 \quad \text { as } \mu_{*} \leq \mu<1 .
\end{aligned}
$$

Thus, the asymmetric equilibria in the region A1 may exist only if $0<\mu<1$ and are determined by the solutions $\tilde{x}$ of (22) as the s follows from (24) agreeing with (25).

Condition (8) for the classical configurations takes the form

$$
\mu+x y>0 .
$$

Inequality (26) then can be rewritten as

$$
x^{2}+y^{2}<1, \quad y \geq-x \quad \text { as }-1<x \leq 0 .
$$

Indeed, since $y<0$ and $x<0$, by multiplying (26) by $y$ and using (23), we get

$$
y(\mu+x y)=x\left(x^{2}-1\right)+x y^{2}=x\left(x^{2}+y^{2}-1\right) \geq 0 \quad \text { or } \quad x^{2}+y^{2} \leq 1 .
$$

For the modified configurations, the inequality sign in (26) changes to the opposite; then the condition of existence is determined by

$$
x^{2}+y^{2}>1, \quad y \geq-x \quad \text { as }-1<x \leq 0 .
$$


On the other hand, by multiplying (26) by $\mu$, one can determine the boundary demarcating the classical configuration from the modified one:

$$
\mu^{2}-x^{2}\left(1-x^{2}\right)=0 .
$$

By solving the biquadratic equation, one can find the intersection points of a unit circle and the cubic parabola of (23):

$$
\hat{x}_{1}(\mu)=-\sqrt{\frac{1}{2}+\sqrt{\frac{1}{4}-\mu^{2}}}, \quad \hat{x}_{2}(\mu)=-\sqrt{\frac{1}{2}-\sqrt{\frac{1}{4}-\mu^{2}}} .
$$

The asymmetric equilibrium is stable if the eigenvalues of the second derivative matrix of the potential $\Pi$ are positive. It can be shown that the eigenvalues are positive if and only if det $\Pi^{\prime \prime}>0$. Moreover, det $\Pi^{\prime \prime}$ coincides with the accuracy of a multiplicator with the derivative of $h(x, \mu)$ over $x$, which leads to

$$
\operatorname{det} \Pi^{\prime \prime}=\frac{\mu}{-x} h^{\prime}(x, \mu) \text {. }
$$

By figuring out $h^{\prime}(x, \mu)$ and omitting always-positive multiplicators, one can see that the equilibrium is stable at the point $\tilde{x}$, the solution of (23), if the function

$$
\Lambda(x, \mu)=\mu x^{2}\left(4 x^{2}-1\right)\left(1-x^{2}\right)+s^{2}(s-\mu)
$$

is positive.

The stability region boundary is determined by $h(x, \mu)=v$ and $h^{\prime}(x, \mu)=0$. However, the condition $h^{\prime}(x, \mu)=0$ implies that the solution $\tilde{x}$ is a local extremum of the function $h(x, \mu)$ and a bifurcation point of the solution of (22), which results in the solution $\tilde{x}$ dividing into the two solutions $\tilde{x}_{-}<\tilde{x}_{+}$. One of the solutions is stable since $h^{\prime}(x, \mu)$ changes its sign at the point $\tilde{x}$. The solutions of $\Lambda(x, \mu)=0$ taking into account the corresponding restrictions on $x$ determine $x$ as a function of $\mu$. Then by substituting it into (22), we have the function $\varrho(\mu)$, whose graph is the boundary of the stability region of the asymmetric equilibria.

The region A1. Equation (22) is written in the form

$$
\mu \frac{s-\mu}{s} k(x)=v .
$$

Since $k(x)<0$, the function $h(x, \mu)$ is positive if $s<\mu$. This inequality is valid if $x^{*}=1 / \sqrt{2}<x<0$. From this, it follows that in the region $A 1$ the solution of (23) lies within the intersection of the interval $\left(x^{*}, 0\right)$ and the intervals determined by the inequalities (25).

In the case of classical configuration, the inequality (27) must be satisfied, while the modified configuration is valid given the inequality (28). The boundary of the solution existence region is determined by the maximal and minimal values of $h(x, \mu)$ for corresponding $\mu$. The stability region is determined by the values 

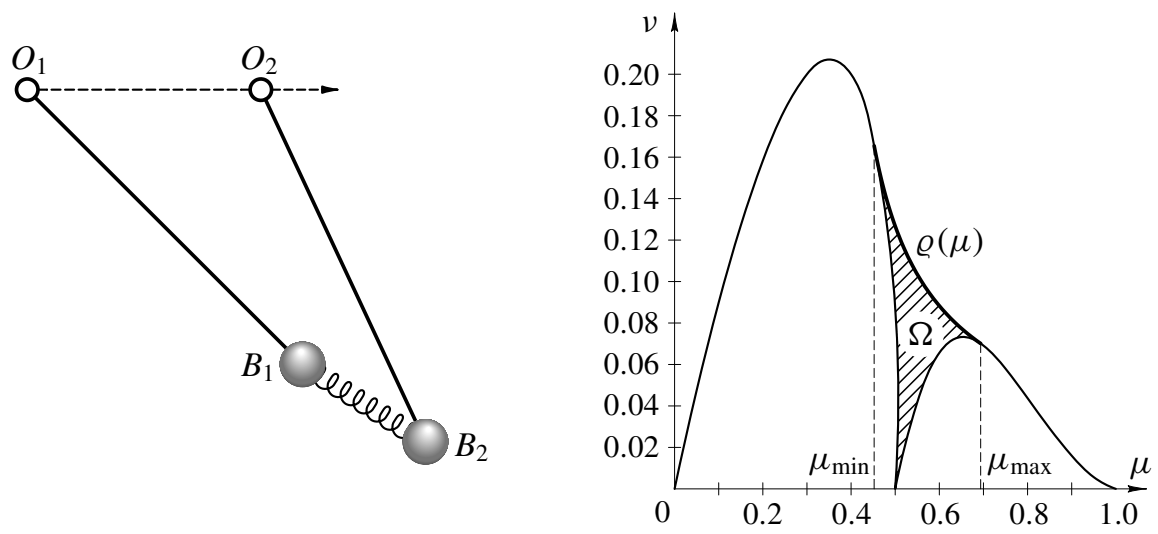

Figure 4. Left: the asymmetric classical configuration in the region $A 1$. Right: the stability domain $\Omega$ of the asymmetric classical configuration in the region $A 1$.

of $\varrho(\mu)$ while $\Lambda(x, \mu)$ must be positive. Figure 4, right, shows the solution existence region of (29) for the sympathetic oscillators (Figure 4, left). The values $\mu_{\min }$ and $\mu_{\max }$ are determined by the condition of maximality and minimality of $\mu$, which ensures $\Lambda(x, \mu)$ to be zero. Calculated values of $\mu_{\min } \approx 0.452258$ and $\mu_{\max } \approx 0.693692$. The stable equilibrium region $\Omega$ is shaded and coincides with the region of two-solution existence $\tilde{x}_{-}<\tilde{x}_{+}$of (22) with $\tilde{x}_{-}$being the stable equilibrium. It is worth noticing that the sympathetic oscillators correspond to the branch of the cubic parabola (23) corresponding to the $x$ satisfying

$$
\hat{x}_{2}(\mu)<x<0 \quad \text { as } 0<\mu<\mu_{*} \quad \text { and } \quad-\sqrt{1-\mu}<x<0 \quad \text { as } \mu_{*} \leq \mu<0 .
$$

The equilibrium existence region of the modified configuration (Figure 5, left, is depicted in Figure 5, right). The condition (28) is satisfied for two branches of the parabola (23) as $0<\mu<\mu_{*}$, corresponding to the $x$ satisfying

$$
-\sqrt{1-\mu} \leq x \leq x_{1}(\mu) \text { and } x_{2}(\mu) \leq x \leq \hat{x}_{2}(\mu) .
$$

Also from the condition $x^{*}<x$, it follows that the first inequality of (30) specifies the modified model in the region $A 1$ as $x^{*}<x_{1}(\mu)$, which is true if $\mu^{*}=1 / 2 \sqrt{2}<\mu$. Given $\mu=\mu_{*}$, these branches coalesce and as $\mu_{*}<\mu$ they specify the sole function $h(x, \mu)$ within the interval $\left(-\sqrt{1-\mu}, \hat{x}_{2}(\mu)\right)$. The condition $-\sqrt{1-\mu}<\hat{x}_{2}(\mu)$ results in the inequality $\mu<\frac{1}{2}$. Therefore, the solution existence region is specified by

$$
\begin{aligned}
x_{2}(\mu) \leq x \leq \hat{x}_{2}(\mu) & \text { as } 0<\mu<\mu_{*}, \\
x^{*} \leq x<\hat{x}_{2}(\mu) & \text { as } \mu_{*} \leq \mu<\frac{1}{2}, \\
x^{*} \leq x<x_{1}(\mu) & \text { as } \mu^{*} \leq \mu<\mu_{*}
\end{aligned}
$$



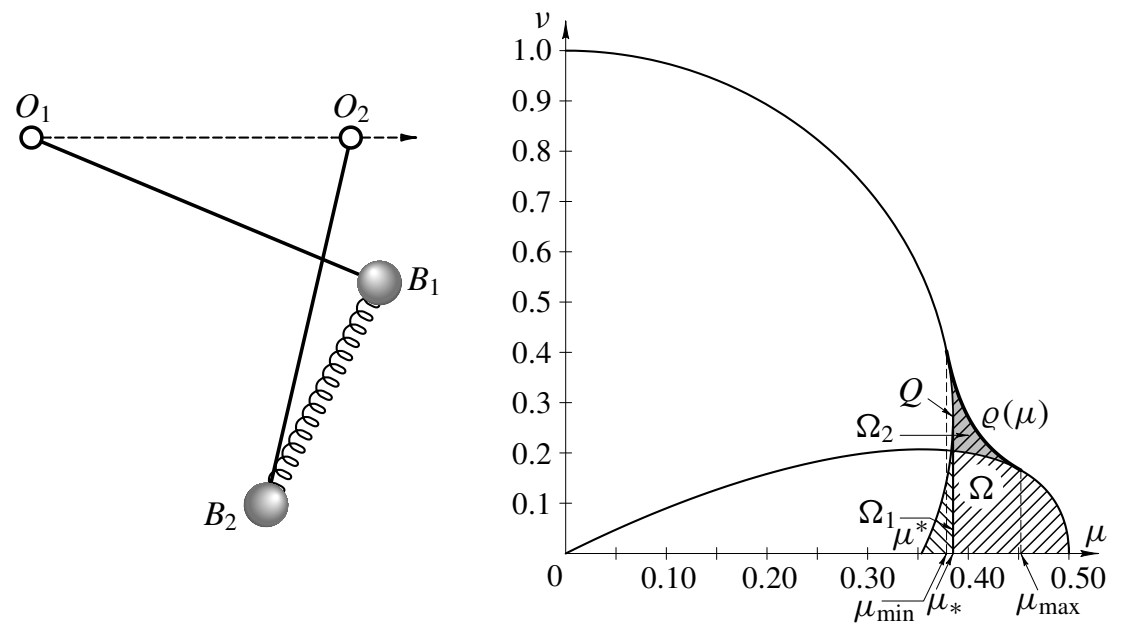

Figure 5. Left: the asymmetrical modified configuration in region A1. Right: the stability domain of the asymmetrical configuration is the merger of the regions $\Omega$ and $\Omega_{1}$.

and bounded by the curves $h\left(\hat{x}_{2}(\mu), \mu\right)$ and $h(-\sqrt{1-\mu}, \mu)$. Analogous to the case of the sympathetic oscillators, one can determine the boundary of the local maximum existence region for the function $h(x, \mu): \mu_{\min } \approx 0.378424$ and $\mu_{\max } \approx$ 0.452258 .

The stability region $\Omega$, corresponding to the branch of the cubic parabola with the point $x_{2}(\mu)$, encompasses the region $\Omega_{2}$ of the two-equilibrium-solution existence. The stability region $\Omega_{1}$ corresponds to the parabola's branch with the point $x_{1}(\mu)$. In the region of two-solution existence, there is a stable equilibrium corresponding to the solution $\tilde{x}_{-}$. The point $Q$ indicates the coalescence point between the branches and equals $(2, \sqrt{2}) / 3 \sqrt{3}$.

The region A2. In this case, we write (22) in the form

$$
\mu \frac{s-\mu}{s} k(x)=-v \text {. }
$$

The solutions of (31) exist if $-1<x<x^{*}$. Since $x^{*} \leq \hat{x}_{2}(\mu)$ and $x^{*} \leq-\sqrt{1-\mu}$, the sympathetic oscillators have no asymmetric equilibria in the region A2.

The modified configurations exist if $s<\mu$ or $x<x^{*}$. This condition is satisfied if $-\sqrt{1-\mu}<x<x_{1}(\mu)$ as $0<\mu<\mu^{*}$ and $-\sqrt{1-\mu}<x<x^{*}$ as $\mu^{*} \leq \mu<\frac{1}{2}$. Since $x<-\frac{1}{2}$ and $s<\mu$, the function $h(x, \mu)$ increases, i.e., $h^{\prime}(x, \mu)>0$. The solution existence region is specified by the inequalities $h(-\sqrt{1-\mu}, \mu)<v<$ $h\left(x_{1}(\mu), \mu\right)$ as $0<\mu<\mu^{*}$ and $h(-\sqrt{1-\mu}, \mu)<v<0$ as $\mu^{*} \leq \mu<\frac{1}{2}$. Since $\operatorname{det} \Pi^{\prime \prime}=v h^{\prime}(x, \mu) / x$ and $x<0$, then $\operatorname{det} \Pi^{\prime \prime}<0$ and there is no stable equilibrium in the region $A 2$. 


\section{Conclusions}

The analysis of the stability of two coupled oscillators showed that the model solutions significantly depend on the dimensionless parameters of varied physical origins. We demonstrated that the natural dimensionless kinematic parameter $\mu$ is subjected to the relation of the distance between the suspension points and the oscillator length. The dimensionless energetic parameter $v$ is equal to the relation between the potential energy of the oscillator and the spring's effective energy. Thus, the parameter set $(\mu, v)$ presents the convenient variables of the model.

Though we considered a static case, dynamic stability of such systems was investigated using chains of particles connected by springs, some of which could exhibit negative stiffness [Pasternak et al. 2014]. The necessary stability condition was formulated: only one spring in the chain can have negative stiffness, and the value of negative stiffness cannot exceed a certain critical value. Applying the Cosserat theory with negative Cosserat shear modulus was proposed in [Pasternak et al. 2016]. It was shown that, when the sum of the negative Cosserat shear modulus and the conventional shear modulus is positive, the waves can propagate.

The demonstrated phenomena of the system's critical dynamics of the linked oscillators are important to general understanding of the nature of different processes. At macroscales, they play a crucial role in determining the fragility and instability of rocks [Tarasov and Guzev 2013] whereas at microscales the dynamics of phononic crystals that are lattices of linked oscillators is governed by the parameters $(\mu, v)$ [Ghasemi Baboly et al. 2013]. In addition, an important application is magnetic tweezers, which may permit us to handle even single micromolecules [Lipfert et al. 2009].

\section{References}

[Arkhipova and Luongo 2014] I. M. Arkhipova and A. Luongo, "Stabilization via parametric excitation of multi-dof statically unstable systems", Commun. Nonlinear Sci. Numer. Simul. 19:10 (2014), 3913-3926.

[Arkhipova et al. 2012] I. M. Arkhipova, A. Luongo, and A. P. Seyranian, "Vibrational stabilization of the upright statically unstable position of a double pendulum", J. Sound Vib. 331:2 (2012), 457469 .

[Butikov 2011] E. I. Butikov, “An improved criterion for Kapitza's pendulum stability”, J. Phys. A 44:29 (2011), 295202.

[dell' Isola et al. 2016] F. dell'Isola, I. Giorgio, M. Pawlikowski, and N. L. Rizzi, "Large deformations of planar extensible beams and pantographic lattices: heuristic homogenization, experimental and numerical examples of equilibrium", P. Roy. Soc. A 472:2185 (2016), 20150790.

[Ghasemi Baboly et al. 2013] M. Ghasemi Baboly, M. F. Su, C. M. Reinke, S. Alaie, D. F. Goettler, I. El-Kady, and Z. C. Leseman, "The effect of stiffness and mass on coupled oscillations in a phononic crystal”, AIP Adv. 3:11 (2013), 112121. 
[Huynh and Chew 2010] H. N. Huynh and L. Y. Chew, "Two-coupled pendulum system: bifurcation, chaos and the potential landscape approach", Internat. J. Bifur. Chaos Appl. Sci. Engrg. 20:8 (2010), 2427-2442.

[Huynh et al. 2013] H. N. Huynh, T. P. T. Nguyen, and L. Y. Chew, "Numerical simulation and geometrical analysis on the onset of chaos in a system of two coupled pendulums", Commun. Nonlinear Sci. Numer. Simul. 18:2 (2013), 291-307.

[Kapitza 1951a] P. L. Kapitza, "Маятник с вибрирующим подвесом" ("Pendulum with vibrating suspension”), Usp. Fiz. Nauk. 44:5 (1951), 7-20.

[Kapitza 1951b] P. L. Kapitza, “Динамическая устойчивость маятника при колеблю ейся точке подвеса", Zh. Eksp. Teor. Fiz. 21:5 (1951), 588-592. Translated as "Dynamical stability of a pendulum when its point of suspension vibrates" pp. 714-725 in Collected papers of P. L. Kapitza, vol. 2, edited by D. ter Haar, Pergamon, London, 1965.

[Kittel 2005] C. Kittel, Introduction to solid state physics, 8th ed., Wiley, Hoboken, NJ, 2005.

[Koluda et al. 2014] P. Koluda, P. Perlikowski, K. Czolczynski, and T. Kapitaniak, "Synchronization configurations of two coupled double pendula", Commun. Nonlinear Sci. Numer. Simul. 19:4 (2014), 977-990.

[Lipfert et al. 2009] J. Lipfert, X. Hao, and N. H. Dekker, "Quantitative modeling and optimization of magnetic tweezers", Biophys. J. 96:12 (2009), 5040-5049.

[Maianti et al. 2009] M. Maianti, S. Pagliara, G. Galimberti, and F. Parmigiani, "Mechanics of two pendulums coupled by a stressed spring", Am. J. Phys. 77:9 (2009), 834-838.

[Markeev 2013] A. P. Markeev, "О движении связанных маятников" (“On the motion of connected pendulums"), Nelin. Dinam. 9:1 (2013), 27-38.

[Pasternak et al. 2014] E. Pasternak, A. V. Dyskin, and G. Sevel, "Chains of oscillators with negative stiffness elements”, J. Sound. Vib. 333:24 (2014), 6676-6687.

[Pasternak et al. 2016] E. Pasternak, A. V. Dyskin, and M. Esin, "Wave propagation in materials with negative Cosserat shear modulus”, Int. J. Eng. Sci. 100 (2016), 152-161.

[Pikovsky and Rosenblum 2015] A. Pikovsky and M. Rosenblum, "Dynamics of globally coupled oscillators: progress and perspectives", Chaos 25 (2015), 097616.

[Ramachandran et al. 2011] P. Ramachandran, S. G. Krishna, and Y. M. Ram, "Instability of a constrained pendulum system", Am. J. Phys. 79:4 (2011), 395-400.

[Seyranian and Seyranian 2008] A. A. Seyranian and A. P. Seyranian, "Chelomei's problem of the stabilization of a statically unstable rod by means of a vibration", J. Appl. Math. Mech. 72:6 (2008), 649-652.

[Sommerfeld 1994] A. Sommerfeld, Vorlesungen über theoretische Physik, Band I: Mechanik, Harri Deutsch, Thun, Switzerland, 1994.

[Stephenson 1908] A. Stephenson, "On induced stability", Philos. Mag. (6) 15:86 (1908), 233-236.

[Tarasov and Guzev 2013] B. G. Tarasov and M. A. Guzev, "Mathematical model of fan-head shear rupture mechanism”, Key Eng. Mat. 592-593 (2013), 121-124.

Received 8 Nov 2015. Revised 11 Apr 2016. Accepted 14 May 2016.

MickHAIL A. GUZEv: guzev@iam.dvo.ru

Institute for Applied Mathematics, Far Eastern Branch, Russian Academy of Sciences, Radio 7, Vladivostok, 690041, Russia

Alexandr A. DMitriev: dmitriev@iam.dvo.ru

Institute for Applied Mathematics, Far Eastern Branch, Russian Academy of Sciences, Radio 7, Vladivostok, 690041, Russia 


\title{
ANALYSIS OF THE ELECTROMAGNETIC REFLECTION AND TRANSMISSION THROUGH A STRATIFIED LOSSY MEDIUM OF AN ELLIPTICALLY POLARIZED PLANE WAVE
}

\author{
FABIO MANGINI AND FABRIZIO FREZZA
}

\begin{abstract}
In this paper, a method to analyze the electromagnetic scattering of an elliptically polarized plane wave through a stratified lossy medium is presented. The interaction of the electromagnetic radiation with the stratified material is taken into account by means of the transfer-matrix approach: in this way, we can consider the stratified medium as an effective single interface. To do that, it was necessary to represent the complex plane-wave propagation vector with two different formulations: the phase and attenuation vectors and the complex angle. Thanks to these two formalisms, it is possible to describe the behaviors of this canonical phenomenon in an elegant way in all the cases of presence of a stratified lossy medium. A numerical code has been implemented to compute the field over the whole space. Finally, to validate the presented model, comparisons with the results presented in the literature have been provided.
\end{abstract}

\section{Introduction}

The determination of the Fresnel coefficients due to the presence of a layered medium with plane interfaces has been the subject of several studies in the last decades because of its important applications, e.g., to forward and inverse scattering by buried two- and three-dimensional objects, to electromagnetic artificial materials, to characterization of porous media and to enhancement of optical antennas and photovoltaic panels [Takenaka et al. 2003; Khoo et al. 2006; Feng et al. 2003; Ziolkowski and Erentok 2006; Taminiau et al. 2008; Frezza et al. 2013; 2015]. We consider the propagation of an inhomogeneous plane wave in a lossy medium, which impinges on the interface with another lossy medium. We assume the media are linear, isotropic, homogeneous, dielectric and generally dispersive and dissipative; with these exceptions, we define the problem in the most general manner. In general, from an electromagnetic point of view, all the materials can be subdivided into three categories: the vacuum with null conductivity $(\sigma=0)$,

\section{Communicated by Francesco dell'Isola.}

MSC2010: 78A25, 78A48.

Keywords: lossy media, transmission and reflection, stratified medium. 


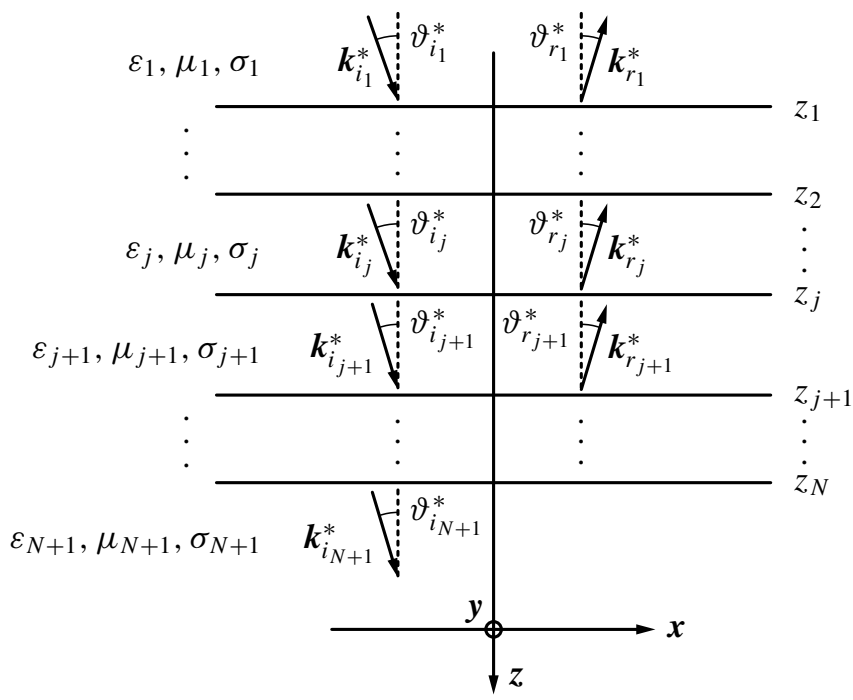

Figure 1. Geometry of the problem with the complex-angle formulation.

absolute dielectric permittivity $\varepsilon=\varepsilon_{0}$ and absolute permeability $\mu=\mu_{0}$; the lossless dielectrics with $\sigma=0, \varepsilon=\varepsilon_{0} \varepsilon_{r}$ and $\mu=\mu_{0} \mu_{r}$; the lossy medium with $\sigma \neq 0$, $\varepsilon=\varepsilon_{0} \varepsilon_{r}$ and $\mu=\mu_{0} \mu_{r}$; and finally the perfect conductor with $\sigma \simeq \infty$. Now we can see the lossy media are the most general materials considerable. In this paper, the complex plane-wave propagation vectors are represented with different formulations: the complex-angle formulation [Ivlev 1987] (Figure 1) and the phase and attenuation vectors, i.e., the Adler-Chu-Fano formulation [Adler et al. 1960] (Figure 2). In Figure 1, considering the $j$-th layer, the impinging complex wave vectors $\boldsymbol{k}_{j}^{*}$ and the transmitted one, $\boldsymbol{k}_{j+1}^{*}$, are shown. The angles that these vectors form with the normal direction (z-axis) to the interfaces are $\vartheta_{j}^{*}$ and $\vartheta_{j+1}^{*}$, respectively. On the other hand, in Figure 2, the phase and attenuation vectors of the incident, $\boldsymbol{\beta}_{j}, \boldsymbol{\alpha}_{j}$, and of the transmitted, $\boldsymbol{\beta}_{j+1}, \boldsymbol{\alpha}_{j+1}$, waves are shown. The angles that these vectors form with the normal direction to the interface are, respectively, $\xi_{j}, \eta_{j}$ and $\xi_{j+1}, \eta_{j+1}$. Moreover, we define the angles between the phase and the attenuation vectors, in the two media, as $\zeta_{j}=\eta_{j}-\xi_{j}$ and $\zeta_{j+1}=\eta_{j+1}-\xi_{j+1}$, respectively. The difference between the complex angle of the transmitted wave vector and the angle of the transmitted phase vector is well understood. However, some confusion between these angles may occur. To emphasize the possible mistake that can be made between these formulations, we consider the arguments in [Canning 2011], where the expressions of the Fresnel coefficients found in the literature are questioned. The mistake has been pointed out and corrected in [Besieris 2011] but allows us to clarify the differences between the possible representations of the 


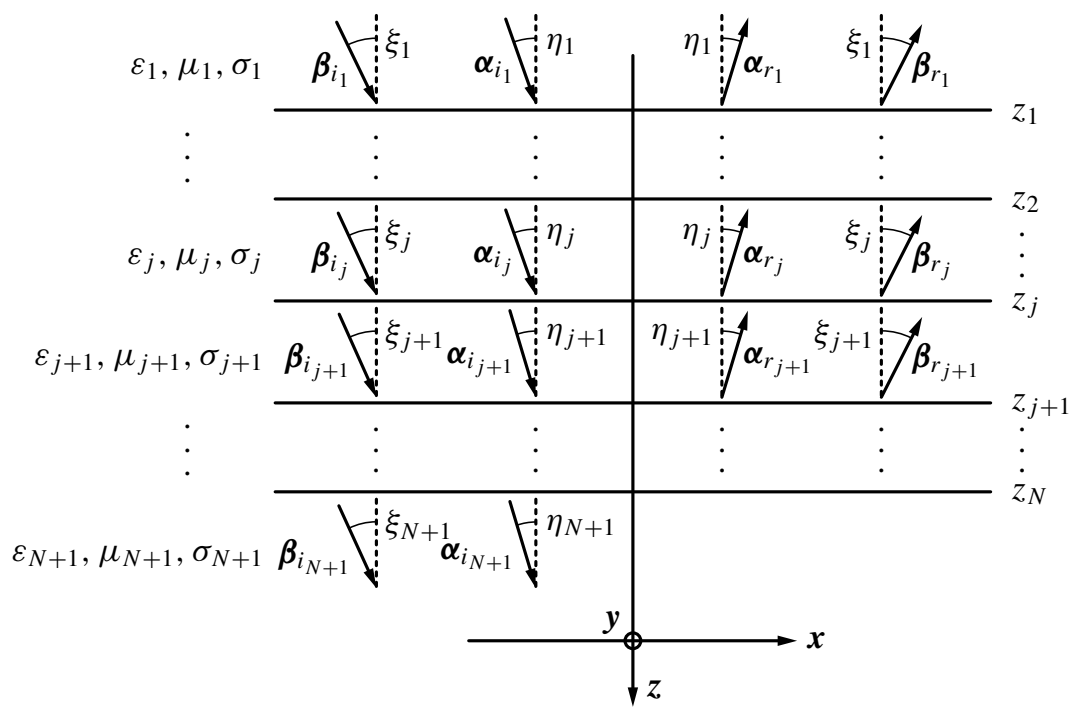

Figure 2. Geometry of the problem with the phase and attenuation vector representation.

complex wave vector of an inhomogeneous plane wave in a lossy medium. Moreover, the connection between these two formulations has been mentioned before, in [Roy 2003], where a numerical result, which needs different determinations in the solution of a polynomial equation of the fourth order and the inversion of a cosine function, is presented.

The two possible representations of a complex wave vector in a lossy medium are very useful to describe the most canonical scenarios about the stratified lossy medium. The complex-angle formulation can be the best solution to represent the transmission wave through a multilayered medium that will impinge on a buried sphere. The actual model of the complex wave vector consists of expanding in two different plane waves and then obtaining a single expansion involving the Wigner 3-j symbols [Kaplan and Resnikoff 1967], i.e., using the Adler-Chu-Fano formulation. Using the complex-angle formulation, it is possible to obtain an expansion of inhomogeneous elliptically polarized plane waves in terms of vectorial spherical harmonics in Mie form just using the Legendre functions generalized via hypergeometrical functions, instead of the classical Legendre functions. Hence, in all cases where a scatterer is present below the stratified lossy medium, in which the scattered electric field has to be represented as an expansion of vectorial harmonics, for example when scatterers with spherical, cylindrical or ellipsoidal shape are present, it is better to use the complex-angle formulation. Indeed, the phase and attenuation vector formulation is indispensable to obtain an elegant model of the classical generalized Fresnel problem. 
In this paper, we consider an elliptically polarized plane wave incident on the stratified material, we show the two possible representations of a complex wave vector in a lossy medium and we use the polarization vectors to calculate the real and imaginary parts of the complex angle as functions of the phase and attenuation vectors. The transmission through the stratified medium is determined by the well known formalism of the transition matrix. This matrix was first introduced by Abelès and is presented in many textbooks of optics and electromagnetics [Abelès 1950; Born and Wolf 1999; Chew 1995]. In the literature, many works have generalized the transmission-matrix method, e.g., with a polynomial expression of its elements [Vigoureux 1991] or by considering anisotropic layers [Essinger-Hileman 2013]. Thanks to the transfer-matrix formalism, the lossy medium stratification can be taken into account, for what concerns our problem, as an effective single interface. In particular, contrary to what is present in the literature, we have obtained an elegant matrix formulation, simply using the combination of the complex-angle formalism and the transfer-matrix approach. Moreover, in that way, we highlight the physical meaning of each element in the transition matrix.

Thanks to the transfer-matrix approach, we can easily extend the number of layers to infinity so as to realize an intermediate layer with peculiar properties. In particular, it will be possible to take into account some materials characterized by a relative permittivity that varies, for example, linearly between two external layers.

In Section 2, the theoretical formulation of the problem is presented in detail. In Section 3, in order to prove the correctness of our method, we show some comparisons with the results presented in the literature. Finally, in Section 4, the conclusions are drawn.

\section{Theoretical Approach}

The geometry of the problem is depicted in Figures 1 and 2. The incident monochromatic radiation on the first surface of the medium is a plane wave traveling from medium 1 to medium $N+1$. Let us call $z$ the stratification direction. Considering the $j$-th interface, the wave vector of the impinging radiation forms an angle $\vartheta_{j}$ with the $z$-axis, and its projection on the interface forms an angle $\varphi_{j}$ with the $x$ axis. Let $\varepsilon_{j}, \mu_{j}$ and $\sigma_{j}$ be the relative permittivity, the relative permeability, and the electrical conductivity in the $j$-th layer of the stratified medium. We remember that all media are linear, isotropic, homogeneous and generally dispersive and dissipative. Then the constitutive equations are

$$
\begin{aligned}
& \boldsymbol{D}(\boldsymbol{r}, \omega)=\varepsilon_{0} \varepsilon_{r} \boldsymbol{E}(\boldsymbol{r}, \omega), \\
& \boldsymbol{B}(\boldsymbol{r}, \omega)=\mu_{0} \mu_{r} \boldsymbol{H}(\boldsymbol{r}, \omega), \\
& \boldsymbol{J}(\boldsymbol{r}, \omega)=\sigma \boldsymbol{E}(\boldsymbol{r}, \omega),
\end{aligned}
$$


where $\omega$ is the angular frequency of the incident field. Solving the Helmholtz equation for the electric field, we can write the incident elliptically polarized field as

$$
\boldsymbol{E}_{i_{1}}(\boldsymbol{r})=\left(E_{i_{1}}^{H} \boldsymbol{\vartheta}_{0 i}+E_{i_{1}}^{E} \boldsymbol{\varphi}_{0 i}\right) e^{i \boldsymbol{k}_{i} \cdot \boldsymbol{r}}
$$

with

$$
\begin{aligned}
\boldsymbol{k}_{i_{1}} & =k_{1}^{*}\left(\sin \vartheta_{i_{1}}^{*} \cos \varphi_{i_{1}} \boldsymbol{x}_{0}+\sin \vartheta_{i_{1}}^{*} \sin \varphi_{i_{1}} \boldsymbol{y}_{0}+\cos \vartheta_{i_{1}}^{*} z_{0}\right), \\
\boldsymbol{\vartheta}_{0 i} & =\cos \vartheta_{i_{1}}^{*} \cos \varphi_{i_{1}} \boldsymbol{x}_{0}+\cos \vartheta_{i_{1}}^{*} \sin \varphi_{i_{1}} \boldsymbol{y}_{0}-\sin \vartheta_{i_{1}}^{*} z_{0}, \\
\boldsymbol{\varphi}_{0 i} & =-\sin \varphi_{i_{1}} \boldsymbol{x}_{0}+\cos \varphi_{i_{1}} \boldsymbol{y}_{0}
\end{aligned}
$$

and with $\boldsymbol{x}_{0}, \boldsymbol{y}_{0}$ and $z_{0}$ the cartesian unit vectors. Throughout this paper, a time dependence $e^{-i \omega t}$ is assumed and always omitted. The reflected $\boldsymbol{E}_{r}(\boldsymbol{r})$ and transmitted $\boldsymbol{E}_{t}(\boldsymbol{r})$ waves by the stratified medium are given by

$$
\begin{aligned}
& \boldsymbol{E}_{r}(\boldsymbol{r})=\left(R_{E}^{H} E_{i_{1}}^{H} \boldsymbol{\vartheta}_{0 r}+R_{E}^{E} E_{i_{1}}^{E} \boldsymbol{\varphi}_{0 r}\right) e^{i \boldsymbol{k}_{r} \cdot \boldsymbol{r}}, \\
& \boldsymbol{E}_{t}(\boldsymbol{r})=\left(T_{E}^{H} E_{i_{1}}^{H} \boldsymbol{\vartheta}_{0 t}+T_{E}^{E} E_{i_{1}}^{E} \boldsymbol{\varphi}_{0 t}\right) e^{i \boldsymbol{k}_{t} \cdot \boldsymbol{r}},
\end{aligned}
$$

where $R_{E}^{H}, T_{E}^{H}$ and $R_{E}^{E}, T_{E}^{E}$ are the effective reflection and transmission coefficients of the stratified medium for parallel $(\mathrm{E})$ and perpendicular $(\mathrm{H})$ polarizations of the electric field, respectively, relevant to the effective interface between medium 1 and medium $N+1$, with the vectors $\boldsymbol{k}_{r}, \boldsymbol{k}_{t}, \boldsymbol{\vartheta}_{0 r}, \boldsymbol{\vartheta}_{0 t}, \boldsymbol{\varphi}_{0 r}$ and $\boldsymbol{\varphi}_{0 t}$ having expressions similar to (5)-(7). For the sake of brevity, we work on the plane $\varphi=0$; however, the following considerations can be easily extended for each plane $\varphi \neq 0$. In this case, the wave number and the complex angle in (5)-(7) can be written as

$$
\begin{aligned}
& k^{*}=k_{\boldsymbol{R}}+i k_{\boldsymbol{I}}, \\
& \vartheta^{*}=\vartheta_{\boldsymbol{R}}+i \vartheta_{\boldsymbol{I}}
\end{aligned}
$$

[Adler et al. 1960]; i.e., it is always possible to represent the wave number and the impinging angle as constituted by a real part and an imaginary part. At the same time, as we can see from Figure 2, the complex wave vector can be represented as

$$
\boldsymbol{k}_{i}=\boldsymbol{\beta}_{i}+i \boldsymbol{\alpha}_{i}
$$

with

$$
\begin{aligned}
\boldsymbol{\beta}_{i} & =\beta_{i}\left(\sin \xi_{i} \boldsymbol{x}_{0}+\cos \xi_{i} z_{0}\right), \\
\boldsymbol{\alpha}_{i} & =\alpha_{i}\left(\sin \eta_{i} \boldsymbol{x}_{0}+\cos \eta_{i} z_{0}\right)
\end{aligned}
$$

being the phase vector and the attenuation vector, respectively. Another representation useful for our treatment is

$$
\begin{aligned}
& \boldsymbol{k}_{i}=k_{i_{\tau}} \boldsymbol{x}_{0}+k_{i_{n}} z_{0}, \\
& \boldsymbol{k}_{r}=k_{r_{\tau}} \boldsymbol{x}_{0}+k_{r_{n}} z_{0}
\end{aligned}
$$


with

$$
\begin{aligned}
& k_{i_{\tau}}=k^{*} \sin \vartheta^{*}=\beta_{i} \sin \xi_{i}+i \alpha_{i} \sin \eta_{i}, \\
& k_{i_{n}}=k^{*} \cos \vartheta^{*}=\beta_{i} \cos \xi_{i}+i \alpha_{i} \cos \eta_{i} ;
\end{aligned}
$$

i.e., we can prefer to divide the wave vector into two components: the tangential one $k_{i_{\tau}}$ and the orthogonal one $k_{i_{n}}$ with respect to the separation interfaces. In order to determine $\beta$ and $\alpha$, we have to impose the well known conditions

$$
\begin{aligned}
\beta^{2}-\alpha^{2} & =k_{\boldsymbol{R}}^{2}-k_{\boldsymbol{I}}^{2}, \\
2 \beta \alpha \cos (\eta-\xi) & =2 k_{\boldsymbol{R}}^{2} k_{\boldsymbol{I}}^{2}
\end{aligned}
$$

[Frezza and Tedeschi 2012]. Solving, we obtain

$$
\begin{aligned}
& \beta=\sqrt{\frac{k_{R}^{2}-k_{I}^{2}}{2}} \sqrt{\sqrt{1+\left[\frac{k_{R} k_{I}}{\left(k_{R}^{2}-k_{I}^{2}\right) \cos \zeta}\right]^{2}}+1}, \\
& \alpha=\sqrt{\frac{k_{R}^{2}-k_{I}^{2}}{2}} \sqrt{\sqrt{1+\left[\frac{k_{R} k_{I}}{\left(k_{R}^{2}-k_{I}^{2}\right) \cos \zeta}\right]^{2}}-1 .}
\end{aligned}
$$

Before proceeding to the study of the reflection and transmission coefficients of the incident field, we want to determine each parameter presented so far; to do that, we can consider the generalized Snell law on the $j$-th interface

$$
\begin{aligned}
\beta_{j} \sin \xi_{j} & =\beta_{j+1} \sin \xi_{j+1}, \\
\alpha_{j} \sin \eta_{j} & =\alpha_{j+1} \sin \eta_{j+1}, \\
\beta_{j+1}^{2}-\alpha_{j+1}^{2} & =k_{\boldsymbol{R}_{j+1}}^{2}-k_{\boldsymbol{I}_{j+1}}^{2}, \\
\beta_{j+1} \alpha_{j+1} \cos \left(\eta_{j+1}-\xi_{j+1}\right) & =2 k_{\boldsymbol{R}_{j+1}}^{2} k_{\boldsymbol{I}_{j+1}}^{2}
\end{aligned}
$$

[Adler et al. 1960]. Using the methodology adopted in [Frezza and Tedeschi 2012], system (23) can be solved, obtaining

$$
\begin{aligned}
& \beta_{j+1}=\sqrt{\frac{\left|k_{i_{j_{\tau}}}\right|^{2}+\left(k_{\boldsymbol{R}_{j+1}}^{2}-k_{\boldsymbol{I}_{j+1}}^{2}\right)+\left|k_{j+1}^{2}-k_{i_{j_{\tau}}}^{2}\right|}{2}}, \\
& \alpha_{j+1}=\sqrt{\frac{\left|k_{i_{j_{\tau}}}\right|^{2}-\left(k_{\boldsymbol{R}_{j+1}}^{2}-k_{\boldsymbol{I}_{j+1}}^{2}\right)+\left|k_{j+1}^{2}-k_{i_{j_{\tau}}}^{2}\right|}{2}}
\end{aligned}
$$


and for the angles

$$
\begin{aligned}
\sin \xi_{j+1} & =\frac{\beta_{j}}{\beta_{j+1}} \sin \xi_{j}, \\
\sin \eta_{j+1} & =\frac{\alpha_{j}}{\alpha_{j+1}} \sin \eta_{j} .
\end{aligned}
$$

Using the generalized Snell law again, we can extend (24) and (25) to the case of stratified media. In particular, the system of equations (23) can be rewritten as

$$
\begin{aligned}
\beta_{1} & =\beta_{N+1} \frac{\sin \xi_{N+1}}{\sin \xi_{1}}, \\
\alpha_{1} & =\alpha_{N+1} \frac{\sin \eta_{N+1}}{\sin \eta_{1}}, \\
\beta_{N+1}^{2}-\alpha_{N+1}^{2} & =k_{\boldsymbol{R}_{N+1}}^{2}-k_{\boldsymbol{I}_{N+1}}^{2}, \\
\beta_{N+1} \alpha_{N+1} \cos \left(\eta_{N+1}-\xi_{N+1}\right) & =2 k_{\boldsymbol{R}_{N+1}}^{2} k_{\boldsymbol{I}_{N+1}}^{2},
\end{aligned}
$$

which once solved gives

$$
\begin{aligned}
& \beta_{N+1}=\sqrt{\frac{\left|k_{i_{\tau}}\right|^{2}+\left(k_{\boldsymbol{R}_{N+1}}^{2}-k_{\boldsymbol{I}_{N+1}}^{2}\right)+\left|k_{N+1}^{2}-k_{i_{1_{\tau}}}^{2}\right|}{2},} \\
& \alpha_{N+1}=\sqrt{\frac{\left|k_{i_{1_{\tau}}}\right|^{2}-\left(k_{\boldsymbol{R}_{N+1}}^{2}-k_{\boldsymbol{I}_{N+1}}^{2}\right)+\left|k_{N+1}^{2}-k_{i_{\tau}}^{2}\right|}{2} .}
\end{aligned}
$$

Now we can see the phase and attenuation constants are independent of the presence of the intermediate layers, as for the case of the lossless scenario. Therefore, the real and the imaginary parts of the complex angle $\vartheta^{*}$ relevant to the last layer may be written as functions of the phase and attenuation vectors:

$$
\begin{aligned}
\cos \vartheta_{\boldsymbol{R}_{N+1}} & =\frac{k_{\boldsymbol{R}_{N+1}} \beta_{N+1} \cos \xi_{N+1}+k_{\boldsymbol{I}_{N+1}} \alpha_{N+1} \cos \eta_{N+1}}{\sqrt{k_{\boldsymbol{R}_{N+1}}^{2} \beta_{N+1}^{2}-k_{\boldsymbol{I}_{N+1}}^{2} \alpha_{N+1}^{2}+2\left(k_{\boldsymbol{R}_{N+1}} k_{\boldsymbol{I}_{N+1}}\right)^{2}}}, \\
\sin \vartheta_{\boldsymbol{R}_{N+1}} & =\frac{k_{\boldsymbol{R}_{N+1}} \beta_{N+1} \sin \xi_{N+1}+k_{\boldsymbol{I}_{N+1}} \alpha_{N+1} \sin \eta_{N+1}}{\sqrt{k_{\boldsymbol{R}_{N+1}}^{2} \beta_{N+1}^{2}-k_{\boldsymbol{I}_{N+1}}^{2} \alpha_{N+1}^{2}+2\left(k_{\boldsymbol{R}_{N+1}} k_{\boldsymbol{I}_{N+1}}\right)^{2}}}, \\
\vartheta_{\boldsymbol{I}_{N+1}} & =\frac{1}{2} \operatorname{atanh}\left(\frac{2 \beta_{N+1} \alpha_{N+1}}{k_{N+1}^{2}}\right) .
\end{aligned}
$$

To determine the value assumed by $\vartheta_{\boldsymbol{R}_{N+1}}$, both (31) and (32) are needed.

We consider a scattering approach to solve the problem; i.e., we take into account all the fields in the different layers and recursively apply the boundary conditions on each interface in order to find the effective reflection and transmission coefficients. Referring to Figure 1, we consider $N+1$ different media, separated 
by $N$ surfaces, each of them identified by the subscript $j$; i.e., we indicate with $\vartheta_{j}, \varphi_{j}, \varepsilon_{j}, \mu_{j}$ and $k_{j}$ the angle of the propagation vector with the $z$-axis, the angle with the $x$-axis of its projection on the $(x, y)$ plane, the relative permittivity, the relative permeability and the wavenumber of the $j$-th medium. Moreover, each layer has thickness $h_{j}$ with the exception of layers 1 and $N+1$, which are two halfspaces. The $j$-th interface separates the $j$-th medium from the $(j+1)$-th medium. Firstly, we consider the parallel (E) polarization; then we will obtain the same result in perpendicular $(\mathrm{H})$ polarization by duality. Our goal is to determine the effective reflection and transmission coefficients for the electric and the magnetic fields $R_{E}^{E}, T_{E}^{E}$ and $R_{H}^{E}, T_{H}^{E}$, respectively, of the structure:

$$
R_{E}^{E}=\frac{E_{r_{1}}^{E}}{E_{i_{1}}^{E}}, \quad T_{E}^{E}=\frac{E_{i_{N+1}}^{E}}{E_{i_{1}}^{E}}, \quad R_{H}^{E}=\frac{H_{r_{1}}^{E}}{H_{i_{1}}^{E}}, \quad T_{H}^{E}=\frac{H_{i_{N+1}}^{E}}{H_{i_{1}}^{E}} .
$$

Looking at the problem from the point of view of multiple reflections on the interfaces between the media, we expect that, in the $j$-th layer, two plane waves propagate: one in the forward direction, with propagation vector $\boldsymbol{k}_{i_{j}}$, that is the superposition of all the secondary reflected waves in the forward direction and the second one in the backward direction, with propagation vector $\boldsymbol{k}_{r_{j}}$, resulting from the superposition of all the secondary reflected waves in the backward direction (see Figure 1). In the last medium, i.e., the $(N+1)$-th layer, there is no backward wave because it is an infinite layer. While the amplitudes of the waves in each layer are our unknowns, the corresponding wave vectors are determined from the Snell law. In fact, similarly to the case of a single dielectric interface, the tangential components of all the wave vectors must be equal to one another: $k_{i_{\tau}}=k_{r_{\tau \tau}}=k_{i_{j+1_{\tau}}}=k_{r_{j+1_{\tau}}}$. From these equalities, we can derive the expressions of the angles $\vartheta_{i_{j}}^{*}$ and $\vartheta_{r_{j}}^{*}$ in each layer. At this point, in order to obtain the coefficients, we have to impose the boundary conditions on each interface. Imposing the continuity of the tangential components of the electric and magnetic field, we obtain

$$
\begin{aligned}
z_{0} \times\left(\boldsymbol{E}_{i_{j}}+\boldsymbol{E}_{r_{j}}-\boldsymbol{E}_{i_{j+1}}-\boldsymbol{E}_{r_{j+1}}\right)=0 & \text { for } z=z_{j}, \\
\boldsymbol{z}_{0} \times\left(\boldsymbol{H}_{i_{j}}+\boldsymbol{H}_{r_{j}}-\boldsymbol{H}_{i_{j+1}}-\boldsymbol{H}_{r_{j+1}}\right)=0 & \text { for } z=z_{j} .
\end{aligned}
$$

The expressions of the electric and magnetic fields for the $j$-th layer are

$$
\begin{aligned}
\boldsymbol{E}_{i_{j}} & =E_{0 i_{j}} \boldsymbol{y}_{0} e^{i\left[k_{i_{j_{\tau}}} x+k_{i_{j_{n}}}\left(z-z_{j}\right)\right]}, \\
\boldsymbol{E}_{r_{j}} & =E_{0 r_{j}} \boldsymbol{y}_{0} e^{i\left[k_{r_{j_{\tau}}} x-k_{r_{j_{n}}}\left(z-z_{j}\right)\right]}, \\
\boldsymbol{H}_{i_{j}} & =\frac{E_{0 i_{j}}}{\omega \mu_{j}}\left(k_{i_{j_{\tau}}} z_{0}-k_{i_{j_{n}}} \boldsymbol{x}_{0}\right) e^{i\left[k_{j_{j_{\tau}}} x+k_{i_{j_{n}}}\left(z-z_{j}\right)\right]}, \\
\boldsymbol{H}_{r_{j}} & =\frac{E_{0 r_{j}}}{\omega \mu_{j}}\left(k_{r_{j_{\tau}}} z_{0}+k_{r_{j_{n}}} \boldsymbol{x}_{0}\right) e^{i\left[k_{r_{j_{\tau}}} x-k_{r_{n}}\left(z-z_{j+1}\right)\right]} .
\end{aligned}
$$


Replacing these expressions in the boundary conditions, we obtain

$$
\left\{\begin{array}{l}
E_{0 i_{j}}+E_{0 r_{j}}=E_{0 i_{j+1}} e^{-i k_{i j+1_{n}}\left(z_{j+1}-z_{j}\right)}+E_{0 r_{j+1}} e^{i k_{i_{j+1}}\left(z_{j+1}-z_{j}\right)}, \\
E_{0 i_{j}}-E_{0 r_{j}}=\zeta_{j, j+1}\left[E_{0 i_{j+1}} e^{-i k_{i_{j+1}}\left(z_{j+1}-z_{j}\right)}-E_{0 r_{j+1}} e^{i k_{i j+1_{n}}\left(z_{j+1}-z_{j}\right)}\right],
\end{array}\right.
$$

having put, for the sake of simplicity, $\zeta_{j, j+1}=\mu_{j} k_{i_{j+1_{n}}} /\left(\mu_{j+1} k_{i_{j_{n}}}\right)$. The linear system (41) can be written in matrix form, yielding

$$
\left[\begin{array}{c}
E_{0 i_{j+1}} \\
E_{0 r_{j+1}}
\end{array}\right]=\left[M_{j}\right]\left[\begin{array}{l}
E_{0 i_{j}} \\
E_{0 r_{j}}
\end{array}\right]
$$

with

$$
\left[M_{j}\right]=\frac{1}{2 \zeta_{j, j+1}}\left[\begin{array}{cc}
\left(1+\zeta_{j, j+1}\right) e^{i \phi_{j+1}} & -\left(1-\zeta_{j, j+1}\right) e^{i \phi_{j+1}} \\
-\left(1-\zeta_{j, j+1}\right) e^{-i \phi_{j+1}} & \left(1+\zeta_{j, j+1}\right) e^{-i \phi_{j+1}}
\end{array}\right],
$$

having put $\phi_{j+1}=k_{i_{j+1_{n}}}\left(z_{j+1}-z_{j}\right)$, the phase difference between two adjacent layers.

We can highlight the term $1+\zeta_{j, j+1}$ :

$$
\left[M_{j}\right]=\frac{1+\zeta_{j, j+1}}{2 \zeta_{j, j+1}}\left[\begin{array}{cc}
e^{i \phi_{j+1}} & -\frac{1-\zeta_{j, j+1}}{1+\zeta_{j, j+1}} e^{i \phi_{j+1}} \\
-\frac{1-\zeta_{j, j+1}}{1+\zeta_{j, j+1}} e^{-i \phi_{j+1}} & e^{-i \phi_{j+1}}
\end{array}\right]
$$

We can note that the term $2 \zeta_{j, j+1} /\left(1+\zeta_{j, j+1}\right)$ is the Fresnel transmission coefficient between the $j$-th and $(j+1)$-th layer $\left(T_{j, j+1}^{E}\right)$ and the term $\left(1-\zeta_{j, j+1}\right) /\left(1+\zeta_{j, j+1}\right)$ is the Fresnel reflection coefficient $\left(R_{j, j+1}^{E}\right)$. So we can rewrite the $\left[M_{j}\right]$ matrix as

$$
\left[M_{j}\right]=\frac{1}{T_{j, j+1}^{E}}\left[\begin{array}{cc}
e^{i \phi_{j+1}} & -R_{j, j+1}^{E} e^{i \phi_{j+1}} \\
-R_{j, j+1}^{E} e^{-i \phi_{j+1}} & e^{-i \phi_{j+1}}
\end{array}\right] .
$$

Now, it is trivial to analyze the transmission through all the layers; in fact, we can write the transmitted field as

$$
\left[\begin{array}{c}
E_{0 i_{N+1}} \\
0
\end{array}\right]=\left[M_{N}\right]\left[\begin{array}{l}
E_{0 i_{N}} \\
E_{0 r_{N}}
\end{array}\right]=\prod_{\ell=1}^{N}\left[M_{\ell}\right]\left[\begin{array}{l}
E_{0 i_{1}} \\
E_{0 r_{1}}
\end{array}\right]=[M]\left[\begin{array}{l}
E_{0 i_{1}} \\
E_{0 r_{1}}
\end{array}\right] .
$$

About the last layer, there is no reflection to obviate this drawback; it is sufficient to place, only for the last layer, $\phi_{N+1}=0$, i.e., consider the last layer to have zero thickness.

Once the overall matrix is obtained, the effective reflection and transmission coefficients of the structure can be found. If we define the transmission matrix as

$$
[M]=\left[\begin{array}{ll}
M_{11} & M_{12} \\
M_{21} & M_{22}
\end{array}\right]
$$


we have

$$
\left\{\begin{array}{l}
M_{11} E_{0 i_{1}}+M_{12} E_{0 r_{1}}=E_{0 i_{N+1}} \\
M_{21} E_{0 i_{1}}+M_{22} E_{0 r_{1}}=0
\end{array}\right.
$$

then the effective coefficients for the parallel polarization are

$$
\begin{aligned}
R_{E}^{E} & =\frac{E_{r_{1}}^{E}}{E_{i_{1}}^{E}}=-\frac{M_{21}}{M_{22}}, \\
T_{E}^{E} & =\frac{E_{i_{N+1}}^{E}}{E_{i_{1}}^{E}}=\frac{M_{11} M_{22}-M_{12} M_{21}}{M_{22}}=\frac{\operatorname{det}[M]}{M_{22}}, \\
R_{H}^{E} & =\frac{H_{r_{1}}^{E}}{H_{i_{1}}^{E}}=\frac{E_{r_{1}}^{E}\left(k_{r_{1}}^{*} / \omega \mu_{1}\right)}{E_{i_{1}}^{E}\left(k_{i_{1}}^{*} / \omega \mu_{1}\right)}=\frac{E_{r_{1}}^{E}}{E_{i_{1}}^{E}}=R_{E}^{E}=-\frac{M_{21}}{M_{22}}, \\
T_{H}^{E} & =\frac{H_{i_{N+1}}^{E}}{H_{i_{1}}^{E}}=\frac{E_{i_{N+1}}^{E}\left(k_{i_{N+1}}^{*} / \omega \mu_{N+1}\right)}{E_{i_{1}}^{E}\left(k_{i_{1}}^{*} / \omega \mu_{1}\right)} \\
& =\frac{\sqrt{\varepsilon_{N+1}} / \sqrt{\mu_{N+1}}}{\sqrt{\varepsilon_{1}} / \sqrt{\mu_{1}}} \frac{E_{i_{N+1}}^{E}}{E_{i_{1}}^{E}}=\frac{Z_{1}^{*}}{Z_{N+1}^{*}} T_{E}^{E}=\frac{Z_{1}^{*}}{Z_{N+1}^{*}} \frac{\operatorname{det}[M]}{M_{22}} .
\end{aligned}
$$

It should be noticed that the matrix $[M]$ that we derived is not the same obtained in the literature [Born and Wolf 1999] but is its inverse: in fact, usually the relation between the transmitted and the incident fields is considered, while we derived the opposite relation.

About the perpendicular polarization, we can find the expression of the coefficients simply exchanging $\zeta_{j, j+1}=\mu_{j} k_{i_{j+1_{n}}} / \mu_{j+1} k_{i_{j_{n}}}$ with $\chi_{j, j+1}=\varepsilon_{j} k_{i_{j+1_{n}}} / \varepsilon_{j+1} k_{i_{j_{n}}}$. It means that the magnetic fields are related by matrices analogous to the ones in (43) but where the parameters $\zeta_{j, j+1}$ must be substituted with $\chi_{j, j+1}$. In this polarization, the transmission matrix assumes the form

$$
\begin{aligned}
{\left[N_{j}\right] } & =\frac{1}{2 \chi_{j, j+1}}\left[\begin{array}{cc}
\left(1+\chi_{j, j+1}\right) e^{i \phi_{j+1}} & -\left(1-\chi_{j, j+1}\right) e^{i \phi_{j+1}} \\
-\left(1-\chi_{j, j+1}\right) e^{-i \phi_{j+1}} & \left(1+\chi_{j, j+1}\right) e^{-i \phi_{j+1}}
\end{array}\right] \\
& =\frac{1}{T_{j, j+1}^{H}}\left[\begin{array}{cc}
e^{i \phi_{j+1}} & -R_{j, j+1}^{H} e^{i \phi_{j+1}} \\
-R_{j, j+1}^{H} e^{-i \phi_{j+1}} & e^{-i \phi_{j+1}}
\end{array}\right],
\end{aligned}
$$

where $T_{j, j+1}^{H}=2 \chi_{j, j+1} /\left(1+\chi_{j, j+1}\right)$ is the transmission coefficient between the $j$-th and $(j+1)$-th layers and the term $R_{j, j+1}^{H}=\left(1-\chi_{j, j+1}\right) /\left(1+\chi_{j, j+1}\right)$ is the reflection coefficient, both in perpendicular polarization. Now the reflection and transmission coefficients of the electric and magnetic fields are

$$
R_{H}^{H}=-\frac{N_{21}}{N_{22}}, \quad T_{H}^{H}=\frac{\operatorname{det}[N]}{N_{22}}, \quad R_{E}^{H}=-\frac{N_{21}}{N_{22}}, \quad T_{E}^{H}=\frac{Z_{N+1}}{Z_{1}} \frac{\operatorname{det}[N]}{N_{22}} .
$$




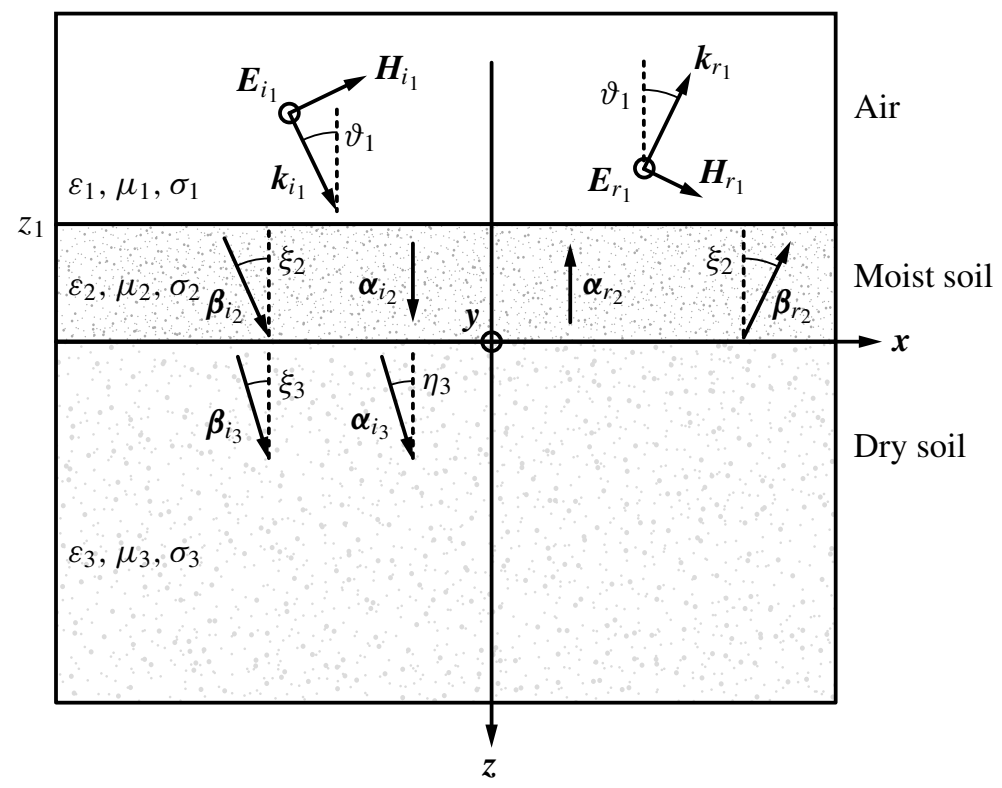

Figure 3. Geometry used to validate the model.

\section{Validation of the model}

To validate our formulation, we compared our results with those of [Curtis 2005], which are based on [Reitz et al. 1979]: their methods exploit an algebraic series that adds up all of the contributions to the net amplitude reflection coefficient of the traveling field in the particular case of just three layers. As a first example, we consider a circular plane wave incident on the stratified medium with the three layers at a frequency of $100 \mathrm{MHz}$ (see Figure 3). The first one is air $\left(\varepsilon_{1}=1\right.$ and $\sigma_{1}=0 \mathrm{~S} / \mathrm{m}$ ), the second one moist soil whose relative dielectric constant is chosen to be $\varepsilon_{2}=10+i 2$, which results in a conductivity of $\sigma_{2}=11.1 \mathrm{mS} / \mathrm{m}$, and the last one dry soil with a complex dielectric constant of $\varepsilon_{3}=3+i 0.2$, which means an effective conductivity of $\sigma_{3}=1.1 \mathrm{mS} / \mathrm{m}$. The thickness of the intermediate layer is $0.05 \mathrm{~m}$ in the first case and $0.20 \mathrm{~m}$ in the second one. In these conditions, we show in Figure 4 the comparison between our results (dashed lines) and the Curtis results (solid line) of the square amplitude of the reflection coefficient in E polarization and $\mathrm{H}$ polarization as a function of the incidence angle: as we can see, the results show a very good agreement in both cases. The second result concerns the square amplitude of the reflection coefficient as a function of the ratio between the toplayer thickness and the top-layer wavelength; the electromagnetic parameters and the geometrical configuration are the same as in the previous case, but now we have considered an incidence angle of 30 degrees (see Figure 5). From the comparisons shown, we can see an optimum agreement, validating our procedure. 

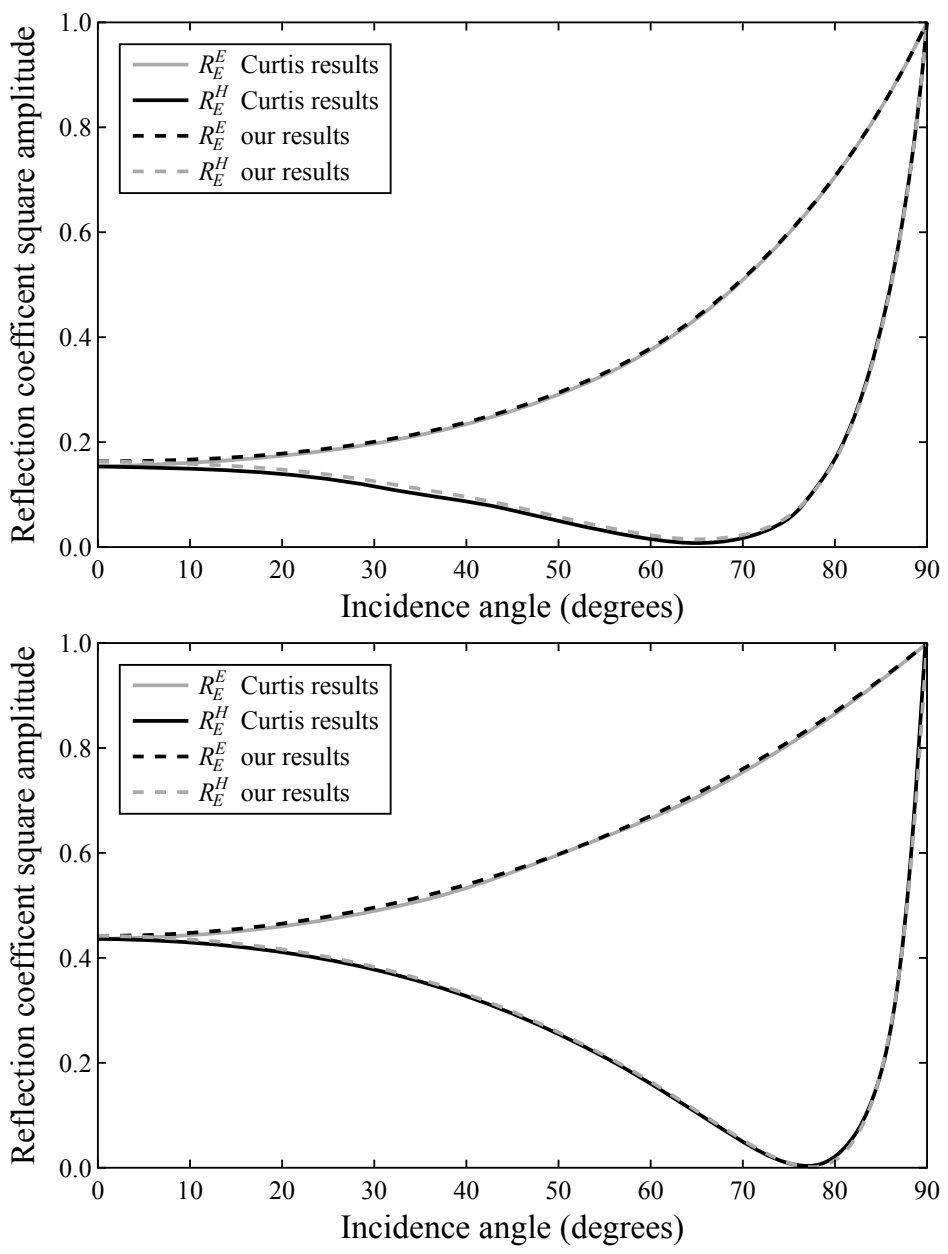

Figure 4. Square amplitude of the reflection coefficient for the electric field as a function of the incidence angle in both polarizations. The stratified medium is located below an air half-space and composed of a layer of moist soil $5 \mathrm{~cm}$ (top) and $20 \mathrm{~cm}$ (bottom) thick on an infinite layer of dry soil.

\section{Conclusion}

In this paper, a rigorous method to solve the electromagnetic scattering problem of an elliptically polarized plane wave by a stratified lossy medium is presented. To determine the reflection and transmission coefficients, we considered the so-called transfer matrix approach. In order to determine each parameter of interest, we have adopted two formalisms: the phase and attenuation vectors and the complex-angle formulation. With these approaches, it is possible to describe all the canonical cases 


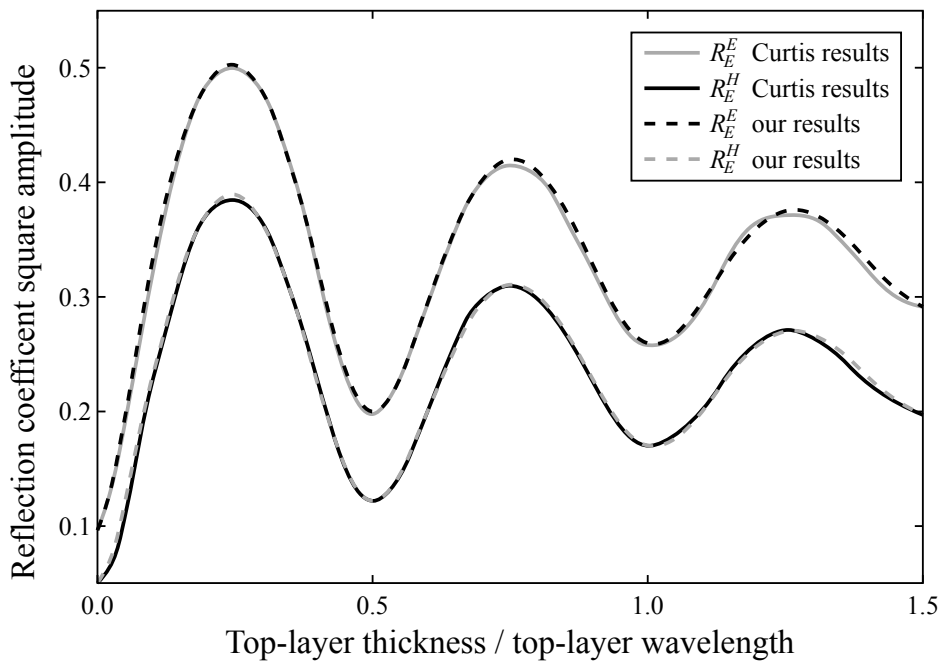

Figure 5. Square amplitude of the reflection coefficient for the electric field as a function of the ratio between the top-layer thickness and the top-layer wavelength in both polarizations with an incident angle of 30 degrees.

in the presence of a multilayered lossy medium, i.e., with or without the presence of a scatterer in the stratified medium. To validate our model, some comparisons with literature results have been presented, obtaining very good agreement in any situation. Obviously this method enjoys the advantages of the transmission-matrix method from which it derives; that is, it can be easily extended to an infinite number of layers to realize an intermediate layer with exotic properties. The generality of the presented method allows its application to several fields of engineering, such as detection of buried or immersed objects, biomedical sensing problems, metamaterial analysis, radar systems, diagnosis of cultural heritage and microscopy; in particular, it can be used to model the lossy medium characterized by a dielectric constant with an anisotropy along the depth direction. Moreover, thanks to this approach, it is possible to design an adaptive material in order to obtain, for example, the zero-reflection and zero-transmission between two external materials: to do this, we can interpose a medium with a relative complex permittivity linearly dependent on the depth.

\section{References}

[Abelès 1950] F. Abelès, "Recherches sur la propagation des ondes électromagnétiques sinusoïdales dans les milieux stratifiés: application aux couches minces”, Ann. Physique (12) 5 (1950), 596-640. [Adler et al. 1960] R. B. Adler, L. J. Chu, and R. M. Fano, Electromagnetic energy transmission and radiation, Wiley, New York, 1960. 
[Besieris 2011] I. M. Besieris, "Comment on the 'Corrected Fresnel coefficients for lossy materials", IEEE Antenn. Propag. M. 53:4 (2011), 161-164.

[Born and Wolf 1999] M. Born and E. Wolf, Principles of optics: electromagnetic theory of propagation, interference and diffraction of light, 7th ed., Cambridge University, 1999.

[Canning 2011] F. X. Canning, "Corrected Fresnel coefficients for lossy materials”, pp. 2123-2126 in 2011 IEEE International Symposium on Antennas and Propagation (Spokane, WA, 2011), IEEE, Piscataway, NJ, 2011.

[Chew 1995] W. C. Chew, Waves and fields in inhomogenous media, IEEE, Piscataway, NJ, 1995.

[Curtis 2005] J. O. Curtis, "A computational tool for simulating plane wave reflectance from layered lossy media", technical report TR-05-3, U.S. Army Corps of Engineers, Engineer Research and Development Center, 2005, Available at http://el.erdc.usace.army.mil/elpubs/pdf/trel05-3.pdf.

[Essinger-Hileman 2013] T. Essinger-Hileman, "Transfer matrix for treating stratified media including birefringent crystals”, Appl. Optics 52:2 (2013), 212-218.

[Feng et al. 2003] Y. Feng, H. Zheng, Z. Zhu, and F. Zu, "The microstructure and electrical conductivity of aluminum alloy foams”, Mater. Chem. Phys. 78:1 (2003), 196-201.

[Frezza and Tedeschi 2012] F. Frezza and N. Tedeschi, "On the electromagnetic power transmission between two lossy media: discussion”, J. Opt. Soc. Am. A 29:11 (2012), 2281-2288.

[Frezza et al. 2013] F. Frezza, F. Mangini, L. Pajewski, G. Schettini, and N. Tedeschi, "Spectral domain method for the electromagnetic scattering by a buried sphere", J. Opt. Soc. Am. A 30:4 (2013), 783-790.

[Frezza et al. 2015] F. Frezza, F. Mangini, and N. Tedeschi, "Electromagnetic scattering by two concentric spheres buried in a stratified material", J. Opt. Soc. Am. A 32:2 (2015), 277-286.

[Ivlev 1987] E. I. Ivlev, "Structure and properties of inhomogeneous waves", J. Mod. Optic. 34:12 (1987), 1559-1569.

[Kaplan and Resnikoff 1967] L. M. Kaplan and M. Resnikoff, "Matrix products and the explicit 3, 6, 9, and 12- $j$ coefficients of the regular representation of $S U(n)$ ", J. Math. Phys. 8:11 (1967), 2194-2205.

[Khoo et al. 2006] I. C. Khoo, D. H. Werner, X. Liang, A. Diaz, and B. Weiner, "Nanosphere dispersed liquid crystals for tunable negative-zero-positive index of refraction in the optical and terahertz regimes", Opt. Lett. 31:17 (2006), 2592-2594.

[Reitz et al. 1979] J. R. Reitz, F. J. Milford, and R. W. Christy, Foundations of electromagnetic theory, 3rd ed., Addison-Wesley, Reading, MA, 1979.

[Roy 2003] J. E. Roy, "New results for the effective propagation constants of nonuniform plane waves at the planar interface of two lossy media", IEEE T. Antenn. Propag. 51:6 (2003), 12061215.

[Takenaka et al. 2003] T. Takenaka, H. Zhou, and T. Tanaka, "Inverse scattering for a three-dimensional object in the time domain", J. Opt. Soc. Am. A 20:10 (2003), 1867-1874.

[Taminiau et al. 2008] T. H. Taminiau, F. D. Stefani, and N. F. van Hulst, "Enhanced directional excitation and emission of single emitters by a nano-optical Yagi-Uda antenna", Opt. Express 16:14 (2008), 10858-10866.

[Vigoureux 1991] J. M. Vigoureux, "Polynomial formulation of reflection and transmission by stratified planar structures", J. Opt. Soc. Am. A 8:11 (1991), 1697-1701.

[Ziolkowski and Erentok 2006] R. W. Ziolkowski and A. Erentok, "Metamaterial-based efficient electrically small antennas”, IEEE T. Antenn. Propag. 54:7 (2006), 2113-2130. 
Received 14 Dec 2015. Revised 17 Feb 2016. Accepted 1 Apr 2016.

FABIO MANGINI: fabio.mangini@uniroma1.it

Information Engineering, Electronics and Telecommunications, Sapienza University of Rome, Via Eudossiana 18, I-00184 Roma, Italy

FABRIZIO FREZZA: fabrizio.frezza@uniroma1.it

Information Engineering, Electronics and Telecommunications, Sapienza University of Rome, Via Eudossiana 18, I-00184 Roma, Italy 



\title{
DISLOCATION-INDUCED LINEAR-ELASTIC STRAIN DYNAMICS BY A CAHN-HILLIARD-TYPE EQUATION
}

\author{
Nicolas Van Goethem
}

\begin{abstract}
In a single crystal containing dislocations, the elastic strain defined by a linear constitutive law from the stress tensor can be written as the sum of a symmetric gradient and a solenoidal tensor $\epsilon^{0}$, called the dislocation strain. This latter part of the elastic strain is related to dislocations since its incompatibility equals the curl of the contortion. The aim of this paper is to derive a time-evolution law for the internal thermodynamic variable $\epsilon^{0}$, arising from the second law of thermodynamics, and to discuss its mathematical setting. This encompasses a discussion on the functional space used and about the equation's well-posedness. A fourth-order time-dependent nonlinear PDE involving the incompatibility operator is found, which is similar in form to the Cahn-Hilliard equation, and represents in this respect a tensor generalization for solenoidal fields.
\end{abstract}

\section{Introduction and preliminary results}

Let $\Omega$ be a simply connected smooth and bounded subset of $\mathbb{R}^{3}$. Let $\mathcal{L}$ be a set of dislocation lines in $\Omega$ and the dislocation density $\Lambda_{\mathcal{L}} \in \mathcal{M}\left(\Omega, \mathbb{M}^{3}\right)$ be given by a Radon measure concentrated in $\mathcal{L}$. As soon as dislocations are present, the strain $\epsilon$ cannot be a symmetric gradient as the following crucial relation, called Kröner's formula, shows [Van Goethem 2016b]:

$$
\text { inc } \epsilon=\operatorname{Curl} \kappa_{\mathcal{L}}, \quad \kappa_{\mathcal{L}}:=\Lambda_{\mathcal{L}}-\frac{\rrbracket_{2}}{2} \operatorname{tr} \Lambda_{\mathcal{L}},
$$

where $\mathbb{I}_{2}$ is the second-rank identity tensor and $\Lambda_{\mathcal{L}}$ the dislocation density tensor defined as $\Lambda_{\mathcal{L}}=\tau \otimes b \mathcal{H}_{L \mathcal{L}}^{1}$ with $\tau$ the tangent vector to the Lipschitz curve $\mathcal{L}$, $\mathcal{H}_{L \mathcal{L}}^{1}$ the one-dimensional Hausdorff measure concentrated in $\mathcal{L}$, and $b$ the Burgers vector, constant on the line. Moreover, inc is the incompatibility operator; i.e.,

$$
\text { inc } F:=\operatorname{Curl~Curl}^{t} F \text {, }
$$

\section{Communicated by Francesco dell'Isola.}

MSC2010: 35J48, 35J50, 35G31, 35K52, 35Q74.

Keywords: dislocations, linear elasticity, incompatibility, Cahn-Hilliard, evolution law, second principle. 
where the curl of a tensor is taken columnwise. This operator is at the heart of the present work since it will be shown to drive the time evolution of the dislocationinduced strain. Note that the evolution of the dislocations are given by the socalled contortion tensor $\kappa_{\mathcal{L}}$, which cannot be determined from the sole knowledge of its curl except in particular cases in which it is divergence-free, as for pure edge dislocations. For this reason, this work is not strictly speaking about the dynamics of dislocations.

Classically in linear elasticity, overall equilibrium reads $\operatorname{div} \mathbb{A} \epsilon=0$ in $\Omega$ with $\mathbb{A}$ the isotropic elasticity tensor. As a consequence, it is shown in [Van Goethem 2015] that there exist two fields of interest: the displacement $u$ and $F$, an auxiliary tensor that is solenoidal and symmetric. These fields satisfy Beltrami decomposition of the elastic strain, namely

$$
\epsilon=\nabla^{S} u+\operatorname{inc} F .
$$

In this paper, our aim is to derive an evolution law for the internal thermodynamic variable

$$
\epsilon^{0}:=\text { inc } F,
$$

which is called the dislocation-induced strain since it satisfies a regularized Kröner's relation inc $\epsilon^{0}=\operatorname{Curl} \kappa$, i.e., has a smoothed dislocation density (namely, the macroscopic contortion $\kappa$ ) in the right-hand side. Furthermore, $\epsilon^{0}$ satisfies a timedependent evolution that turns out to be sufficient for the global mechanical dissipation to be positive.

Specifically in this paper, we establish in a first step, study in a second step, and eventually discuss the nonlinear tensor-valued equation

$$
\alpha \partial_{t} \epsilon^{0}=\operatorname{inc}\left(-\mathbb{M} \text { inc } \epsilon^{0}-\mathcal{G}\left(\epsilon^{0}\right)\right) \quad \text { in } \Omega \times[0, T]
$$

with $\mathcal{G}$ a nonlinear potential, $\alpha>0$, and $\mathbb{M}$ a positive-definite and symmetric fourthorder material-dependent tensor. For simplicity, and for the sake of physical interpretation, we assume that $\mathcal{G}$ depends only on $e:=\operatorname{tr} \epsilon^{0}$, the trace of the dislocationinduced strain, which is shown to be directly related to and hence interpreted as a density of point defects. To achieve this goal, the mathematical nature of the incompatibility operator must be understood, and hence, a series of mathematical results must be recalled as preliminary steps.

Observe that evolution law (1-1) has a form similar to the Cahn-Hilliard equation but for a tensor-valued unknown $\epsilon^{0}$. Indeed, the Laplacian counterpart is precisely the incompatibility operator since $\operatorname{tr} \operatorname{inc} F=\Delta \operatorname{tr} F$, and hence, (1-1) appears as a tensor generalization for solenoidal tensor fields of the classical scalar Cahn-Hilliard equation. From a physical point of view, the scalar version of our equation is related to the dynamics of point defects, which are required for the creation and motion of dislocations and are related to the variation of matter density. 
Furthermore, $e$ obeys the scalar Cahn-Hilliard equation, though with nonstandard boundary conditions. A discussion about this equation, though derived by other means and with a different purpose, can be found in [Van Goethem 2014]. The purpose of this paper is to show that this equation is well-posed in an appropriate functional space; some of its important properties are given. Let us emphasize that particular care is given to justify the equation boundary conditions, which must be mathematically sound and at the same time have a physical interpretation.

The notion of internal variable of state. We consider $F$ a mathematical gauge field arising from Beltrami decomposition of symmetric tensors and without any particular physical meaning. However, its incompatibility, $\epsilon^{0}:=\operatorname{inc} F$, is the dislocation-induced strain since it is the only part of the elastic strain which appear in Kröner's formula. It is considered an internal variable of state (IVS) in the sense given here by G. Maugin [2015]: "Internal variables of state are introduced in thermomechanics in addition to the usual observable variables of state (e.g., deformation, temperature, electric, and magnetic fields). They are supposed to account in a more or less crude way for the complex internal microscopic processes that occur in the material and manifest themselves at a macroscopic scale in the form of dissipation".

Motivation. In our case, the observable variable of state (OVS) is the stress $\sigma$, from which the elastic strain $\epsilon$ is deduced by a constitutive law (hence, the latter is also an OVS). So far, $u$ and $F$ are vector and tensor fields involved in the decomposition of $\epsilon$. In some sense, $u$ is also observable, measurable, and controllable, depending on its boundary conditions and on the introduction of a reference configuration, which is an uncomfortable notion in infinitesimal elasticity. As a matter of fact, we prefer to let the identification $u$ be the displacement field as a convenient "vue de l'esprit".

The crucial point is that $\epsilon^{0}$ is an internal variable that is neither observable nor measurable or controllable, in the sense of physicists. Only its existence as a mathematical object and its effect in the form of dissipation is observed. Therefore, the aim of this paper is to show that it naturally obeys a PDE and thus becomes observable, measurable, and controllable in a mathematical sense. It should be emphasized that there exists no consensual procedure in the literature to determine the equation governing an IVS. Our plan is to derive such an equation in the simplest and most natural possible way, while not contradicting (at least) or, better, complying with (so far as possible) thermodynamics principles.

Structure of the work. The main part of this paper is about the derivation of the incompatibility-governed time-dependent model for the dislocation strain. To this end, considerations about the statics problem, and in particular about the choice 
of the boundary conditions and their physical meaning, are found in Sections 3, 3.1, and 3.2, respectively. The evolution law is then found in Section 4, whose mathematical properties, such as existence of solutions and energy bounds, are given in Section 5. In the preliminary Section 2, the functional spaces needed to mathematically handle the incompatibility operator are given. Several properties of tensor-valued fields with bounded incompatibility are also recalled, without proofs, to be found in a specifically dedicated paper [Amstutz and Van Goethem 2016]. A discussion is given in Section 6.

Notation and conventions. Let $E \in \mathbb{S}^{3}$ and $\beta \in \mathbb{M}^{3}$, where $\mathbb{M}^{3}$ denotes the space of square 3-matrices and $\mathbb{S}^{3}$ of symmetric 3-matrices. Note that superscript $t$ stands for the transpose of a tensor and superscript $S$ for the symmetric part of a tensor. The divergence and curl of a tensor $E$ are defined componentwise as (div $E)_{i}:=$ $\partial_{j} E_{i j}$ and $(\operatorname{Curl} E)_{i j}:=\epsilon_{j k l} \partial_{k} E_{i l}$, respectively. The incompatibility of a tensor $E$ is the symmetric tensor defined componentwise as

$$
(\operatorname{inc} E)_{i j}:=\left(\operatorname{Curl~Curl}^{t} E\right)_{i j}=\epsilon_{i k m} \epsilon_{j l n} \partial_{k} \partial_{l} E_{m n}=\left(\operatorname{Curl}^{t} \operatorname{Curl}^{t} E\right)_{i j} .
$$

Also, $(E \times N)_{i j}=-(N \times E)_{i j}=-\epsilon_{j k m} N_{k} E_{i m}$. Moreover, $\int_{\Omega} \operatorname{Curl} F \cdot E d x=$ $\int_{\Omega} F \cdot \operatorname{Curl} E d x$ and $\int_{\Omega}$ inc $F \cdot E d x=\int_{\Omega} F \cdot$ inc $E d x$ for smooth tensor-valued functions $E$ and $F$ with compact support in $\Omega$. It is a key part of this paper (see Section 2.1) to determine appropriate boundary conditions in order for this integration by parts to be valid for more general fields. We will also use the shorthand

$$
a \mid b:=\int_{\Omega} a \cdot b d x
$$

The following theorem is crucial for the developments of this work.

Theorem 1 (Beltrami decomposition [Maggiani et al. 2015]). Let $\Omega \subseteq \mathbb{R}^{3}$ be a simply connected domain with smooth boundary, let $p \in(1,+\infty)$ be a real number, and let $E \in L^{p}\left(\Omega, \mathbb{S}^{3}\right)$ be a symmetric tensor. Then there exist a vector field $u \in W^{1, p}\left(\Omega, \mathbb{R}^{3}\right)$ and a tensor $F \in L^{p}\left(\Omega, \mathbb{S}^{3}\right)$ with $\operatorname{Curl} F \in L^{p}\left(\Omega, \mathbb{S}^{3}\right)$, inc $F \in$ $L^{p}\left(\Omega, \mathbb{S}^{3}\right), \operatorname{div} F=0$ in $\Omega$, and $F N=0$ on $\partial \Omega$, where $N$ stands for the unit normal to $\partial \Omega$, satisfying

$$
E=\nabla^{S} u+\operatorname{inc} F
$$

Moreover, $u$ can be taken with vanishing trace on $\partial \Omega$, and such a pair $(u, F)$ is unique. 


\section{Preliminary results: functional spaces}

Define

$$
\begin{aligned}
H_{\text {curl }}\left(\Omega ; \mathbb{M}^{3}\right) & :=\left\{E \in L^{2}\left(\Omega ; \mathbb{M}^{3}\right): \operatorname{Curl} E \in L^{2}\left(\Omega, \mathbb{M}^{3}\right)\right\}, \\
\mathcal{H}(\Omega) & :=\left\{E \in H^{2}\left(\Omega, \mathbb{S}^{3}\right): \operatorname{div} E=0\right\}, \\
\mathcal{H}_{0}(\Omega) & :=\left\{E \in \mathcal{H}(\Omega): E=\operatorname{Curl}^{t} E \times N=0 \text { on } \partial \Omega\right\} .
\end{aligned}
$$

These spaces are naturally endowed with the Hilbertian structure of $H^{2}\left(\Omega, \mathbb{S}^{3}\right)$.

Some identities in the local basis. Let us consider the local orthonormal basis $\left(\tau^{A}, \tau^{B}, N\right)$ on $\partial \Omega$ (for detail on such bases and their extension in $\Omega$, see [Amstutz and Van Goethem 2016]). For a general symmetric tensor $T$, one has in this basis

$$
\begin{gathered}
T=\left(\begin{array}{lll}
T_{A A} & T_{A B} & T_{A N} \\
T_{B A} & T_{B B} & T_{B N} \\
T_{N A} & T_{N B} & T_{N N}
\end{array}\right), \quad T \times N=\left(\begin{array}{lll}
T_{A B} & -T_{A A} & 0 \\
T_{B B} & -T_{B A} & 0 \\
T_{N B} & -T_{N A} & 0
\end{array}\right), \\
(T \times N)^{t} \times N=\left(\begin{array}{ccc}
T_{B B} & -T_{A B} & 0 \\
-T_{A B} & T_{A A} & 0 \\
0 & 0 & 0
\end{array}\right) .
\end{gathered}
$$

By the same token,

and

$$
\begin{aligned}
& \left(T \times \tau^{A}\right)^{t} \times \tau^{A}=\left(\begin{array}{ccc}
0 & 0 & 0 \\
0 & T_{N N} & -T_{B N} \\
0 & -T_{N B} & T_{B B}
\end{array}\right), \\
& \left(T \times \tau^{B}\right)^{t} \times \tau^{B}=\left(\begin{array}{ccc}
T_{N N} & 0 & -T_{A N} \\
0 & 0 & 0 \\
-T_{N A} & 0 & T_{A A}
\end{array}\right),
\end{aligned}
$$

$$
\begin{aligned}
& \left(T \times \tau^{A}\right)^{t} \times \tau^{B}=\left(\begin{array}{ccc}
0 & 0 & 0 \\
-T_{N N} & 0 & T_{A N} \\
T_{N B} & 0 & -T_{A B}
\end{array}\right), \\
& \left(T \times \tau^{B}\right)^{t} \times \tau^{A}=\left(\begin{array}{ccc}
0 & -T_{N N} & T_{B N} \\
0 & 0 & 0 \\
0 & T_{N A} & -T_{B A}
\end{array}\right) .
\end{aligned}
$$

Similarly,

$$
\begin{aligned}
& (T \times N)^{t} \times \tau^{A}=\left(\begin{array}{ccc}
0 & T_{N B} & -T_{B B} \\
0 & -T_{N A} & T_{B A} \\
0 & 0 & 0
\end{array}\right), \\
& (T \times N)^{t} \times \tau^{B}=\left(\begin{array}{ccc}
-T_{N B} & 0 & T_{A B} \\
T_{N A} & 0 & -T_{A A} \\
0 & 0 & 0
\end{array}\right) .
\end{aligned}
$$


2.1. Green formula for the incompatibility operator. Let $V$ be a vector field defined on $\partial \Omega$, and let $\widetilde{V}$ be any extension of $V$ in $\Omega$ with appropriate regularity. The surface divergence of $V$ is defined on $\partial \Omega$ by

$$
\operatorname{div}_{S} V=\operatorname{div} \widetilde{V}-\left(\partial_{N} \widetilde{V}\right) \cdot N .
$$

The following result holding for smooth boundaries is sufficient for our purposes whereas, if the boundary had edges, an additional line-integral term must be added. Lemma 2 (surface divergence [Henrot and Pierre 2005]). If $V \in W^{1,1}\left(\partial \Omega, \mathbb{R}^{3}\right)$, then

$$
\int_{\partial \Omega} \operatorname{div}_{S} V d S(x)=\int_{\partial \Omega} \kappa V \cdot N d S(x) .
$$

Lemma 3 [Amstutz and Van Goethem 2016]. For all $U, V \in \mathcal{C}^{2}\left(\bar{\Omega}, \mathbb{M}^{3}\right)$,

$$
\int_{\Omega} U \cdot \operatorname{Curl} V d x=\int_{\Omega} \operatorname{Curl} U \cdot V d x+\int_{\partial \Omega}(U \times N) \cdot V d S(x) .
$$

Denote by $U^{S}=\left(U+U^{t}\right) / 2$ the symmetric part of a tensor $U$, and recall the definition of incompatibility (1-2). The following result is about integration by parts.

Lemma 4 [Amstutz and Van Goethem 2016]. Suppose that $T \in \mathcal{C}^{2}\left(\bar{\Omega}, \mathbb{S}^{3}\right)$ and $\eta \in H^{2}\left(\Omega, \mathbb{S}^{3}\right)$. Then

$$
\int_{\Omega} T \cdot \operatorname{inc} \eta d x=\int_{\Omega} \operatorname{inc} T \cdot \eta d x+\int_{\partial \Omega} \mathcal{T}_{1}(T) \cdot \eta d S(x)+\int_{\partial \Omega} \mathcal{T}_{0}(T) \cdot \partial_{N} \eta d S(x)
$$

with the trace operators defined as

$$
\begin{aligned}
& \mathcal{T}_{0}(T):=(T \times N)^{t} \times N, \\
& \mathcal{T}_{1}(T):=\left(\operatorname{Curl}(T \times N)^{t}\right)^{S}+\left(\left(\partial_{N}+\kappa\right) T \times N\right)^{t} \times N+\left(\operatorname{Curl}^{t} T \times N\right)^{S} .
\end{aligned}
$$

Remark 5. Only $\left(\partial_{N} \eta\right)_{T}$ matters in the rightmost integral of (2-7) since it can be equivalently rewritten as $\int_{\partial \Omega} \mathcal{T}_{0}(T) \cdot \mathcal{T}_{0}\left(\partial_{N} \eta\right) d S(x)$.

Remark 6. Let $\kappa^{R}$ be the two principal curvatures of $\partial \Omega$. It has been proved in [Amstutz and Van Goethem 2016] that ${ }^{1}$

$$
\operatorname{Curl}(T \times N)^{t}=-\sum_{R} \kappa^{R}\left(T \times \tau^{R}\right)^{t} \times \tau^{R}+\left(\operatorname{Curl}^{t} T \times N\right)^{t} .
$$

Taking an $\eta$ such that $\eta N=0=\partial_{N} \eta$ on $\partial \Omega$, then the boundary terms in (2-7) can be rewritten as

$$
\int_{\partial \Omega} \mathcal{T}_{1}(T) \cdot \eta d S(x)=\int_{\partial \Omega} \mathcal{T}_{1}(T)_{T} \cdot \eta_{T} d S(x)
$$

\footnotetext{
${ }^{1}$ The coefficient $\xi$ in [Amstutz and Van Goethem 2016] can be taken to be vanishing.
} 
Now, assuming that $\left(\operatorname{Curl}^{t} T \times N\right)^{S}=0$ and that $\mathcal{T}_{0}(T)=\mathcal{T}_{0}\left(\partial_{N} T\right)=0$ on $\partial \Omega$, taking into account (2-3), (2-9), and (2-10), the second Neumann boundary conditions, due to $(2-11)$, are

$$
\mathcal{T}_{1}(T)_{T}=-T_{N N} D N=0 \quad \text { on } \partial \Omega \text { where } D N=\left(\begin{array}{cc}
\kappa^{A} & 0 \\
0 & \kappa^{B}
\end{array}\right),
$$

with $T_{N N}=T N \cdot N$. In summary, we have the implication

$\left(\operatorname{Curl}^{t} T \times N\right)^{S}=\mathcal{T}_{0}(T)=\mathcal{T}_{0}\left(\partial_{N} T\right)=T N \cdot N=0 \quad$ and $\quad \eta N=0 \quad$ on $\partial \Omega$

$$
\Rightarrow \mathcal{T}_{1} \cdot \eta(T)=0 \text {. }
$$

Remark 7. The following alternative expression is also established in [Amstutz and Van Goethem 2016]:

$$
\begin{aligned}
\mathcal{T}_{1}(T)=-\sum_{R} \kappa^{R}\left(T \times \tau^{R}\right)^{t} \times \tau^{R}+\left(\left(-\partial_{N}+\kappa\right)\right. & T \times N)^{t} \times N \\
& -\sum_{R}\left(\partial_{R} T \times N\right)^{t} \times \tau^{R},
\end{aligned}
$$

where $\tau_{R}$ stands for the derivative along the $R$-th tangent vector $\tau^{R}$, for $R=A$ or $B$. Note that (2-14) is proved in [Amstutz and Van Goethem 2016, Lemma 3.19] by taking $\xi=0$ (since we consider smooth surfaces without umbilical points) and noting that each term of (2-14) is symmetric.

2.2. Basic properties. The following lemma is easy to prove from the properties of these functions.

Lemma 8. Every $E \in \mathcal{H}_{0}(\Omega)$ satisfies $\operatorname{div} \operatorname{Curl}^{t} E=0$ in $\Omega$ and $\operatorname{Curl}^{t} E \times N=$ $\partial_{N} E \times N=0$ on $\partial \Omega$. Moreover, inc $E|F=E|$ inc $F$ for every $E, F \in \mathcal{H}_{0}(\Omega)$.

Proof. The first statement comes easily from the solenoidal property of $E$. As for the second, compute componentwise (see [Amstutz and Van Goethem 2016] for detail)

$$
-\left[\operatorname{Curl}^{t} E \times N\right]_{m q}=\left(\left(\partial_{N} E \times N\right)^{t} \times N\right)_{m q}-\left(\left(\sum_{R} \tau^{R} \times \partial_{R} E\right)^{t} \times N\right)_{m q},
$$

where $\partial_{R}$ means the $R$-th tangential derivative, which here vanishes identically, proving the result. The last statement is a direct consequence of Lemma 4 and Remark 5, and taking into account the density of smooth functions in $\mathcal{H}_{0}(\Omega)$.

Lemma 9. Let $\Omega \subset \mathbb{R}^{3}$ be a bounded open set with boundary of class $\mathscr{C}^{1}$ and $F \in H_{\text {curl }}\left(\Omega ; \mathbb{M}^{3}\right)$ such that $F \times N=0$ on $\partial \Omega$. Then $(\operatorname{Curl} F) N=0$ on $\partial \Omega^{2}{ }^{2}$ Moreover, (inc $E) N=0$ on $\partial \Omega$ as soon as $E=\left(\partial_{N} E \times N\right)^{t} \times N=0$ on $\partial \Omega$.

\footnotetext{
${ }^{2}$ This expression is intended in a classical weak sense; see, e.g., [Van Goethem 2015].
} 
Proof. The first part is proven by taking an arbitrary $\varphi \in H^{2}\left(\Omega, \mathbb{R}^{3}\right)$ since by integration by parts and Lemma $3\langle(\operatorname{Curl} F) N, \varphi\rangle_{\partial \Omega}=\langle\operatorname{Curl} F, D \varphi\rangle=\langle F \times N, D \varphi\rangle_{\partial \Omega}=0$. The second part follows from the first part, the definition of incompatibility, and the identity $0=\operatorname{Curl}^{t} F \times N=0$ from Lemma 8 .

For a proof of the next lemma, see, e.g., [Kozono and Yanagisawa 2009; von Wahl 1992; Bolik and von Wahl 1997].

Lemma 10. Let $F \in H_{\text {curl }}\left(\Omega ; \mathbb{M}^{3}\right)$ such that $\operatorname{div} F=0$ in $\Omega$ and $F \times N=0$ on $\partial \Omega$. Then $F \in H^{1}\left(\Omega, \mathbb{M}^{3}\right)$, and

$$
\|\nabla F\|_{L^{2}(\Omega)} \leq C\|\operatorname{Curl} F\|_{L^{2}(\Omega)} .
$$

The next result follows without major difficulty from Lemma 10.

Lemma 11. For all $E \in \mathcal{H}_{0}(\Omega)$,

$$
\|E\|_{H^{2}(\Omega)} \leq C\left(\|E\|_{L^{2}(\Omega)}+\|\operatorname{Curl} E\|_{L^{2}(\Omega)}+\|\operatorname{inc} E\|_{L^{2}(\Omega)}\right) .
$$

The following theorem is nonclassical but also easy to prove.

Theorem 12 (Poincaré). Let $\partial \Omega_{0} \subset \partial \Omega$ be nonflat with $\mathcal{H}^{2}\left(\partial \Omega_{0}\right)>0$. There exists a constant $C>0$ such that, for each $u \in H^{1}\left(\Omega ; \mathbb{R}^{3}\right)$,

$$
\|u\|_{L^{2}(\Omega)} \leq C\left(\|\nabla u\|_{L^{2}(\Omega)}+\int_{\partial \Omega_{0}}|u \times N| d S\right) .
$$

Theorem 13 (coercivity [Amstutz and Van Goethem 2016]). Let $\Omega$ be a bounded and connected domain with $\mathcal{C}^{1}$ boundary, and let the nowhere-flat subset $\partial \Omega_{0} \subset \partial \Omega$ with $\mathcal{H}^{2}\left(\partial \Omega_{0}\right)>0$. There exists a constant $C>0$ such that, for each $E \in \mathcal{H}_{0}(\Omega)$,

$$
\|E\|_{H^{2}(\Omega)} \leq C \| \text { inc } E \|_{L^{2}(\Omega)} .
$$

\section{Kinematics with dislocations}

First, the complete equations deriving from conservation of momentum are provided. They turn out to be nonclassical since, in the presence of dislocations, an auxiliary tensor variable appears as well as a dislocation-induced force in the righthand side of the equilibrium equation. Second, we discuss the chosen boundary conditions from a mathematical and physical standpoint. Let us emphasize here that from now on the forces will be regularized so that all fields are assumed smooth. This will allow us to perform a thermodynamical study in a classical manner.

3.1. Governing PDEs. The elastic strain is given from the stress tensor $\sigma$ by $\epsilon:=$ $\mathbb{A}^{-1} \sigma$, where $\mathbb{A}$ is the assumed constant elasticity tensor, i.e., $\mathbb{A}=2 \mu \rrbracket_{4}+\lambda \rrbracket_{2} \otimes \mathbb{I}_{2}$, 
where $\mathbb{\square}_{4}$ and $\mathbb{\rrbracket}_{2}$ are the fourth- and second-rank identity tensors, respectively, ${ }^{3}$ with $\mu$ and $\lambda$ the Lamé coefficients. Conservation of linear momentum reads

$$
\left\{\begin{aligned}
\rho \frac{d v}{d t}-\operatorname{div} \mathbb{A} \epsilon & =f & & \text { in } \Omega, \\
\sigma N & =g & & \text { on } \partial \Omega,
\end{aligned}\right.
$$

where $\rho$ is the volumic mass and $v$ the velocity and with $f \in \mathscr{C}^{\infty}\left(\Omega, \mathbb{R}^{3}\right)$ and $g \in \mathscr{C}^{\infty}\left(\partial \Omega, \mathbb{R}^{3}\right)$ the volume and surface forces, respectively. By Beltrami decomposition (see Theorem 1), there exists a vector $u$ and a symmetric and solenoidal tensor $F$ such that

$$
\epsilon=\nabla^{S} u+\text { inc } F
$$

whereby, recalling the solenoidal property of

$$
\epsilon^{0}:=\text { inc } F \text {, }
$$

conservation of linear momentum is rewritten as

$$
\left\{\begin{aligned}
\rho \frac{d v}{d t}-\operatorname{div}\left(\mathbb{A} \nabla^{S} u\right) & =\mathcal{F}_{\mathcal{L}}:=f+\lambda \nabla \operatorname{tr}(\operatorname{inc} F) & & \text { in } \Omega, \\
\left(\mathbb{A} \nabla^{S} u\right) N & =g-\lambda \operatorname{tr}(\operatorname{inc} F) N & & \text { on } \partial \Omega .
\end{aligned}\right.
$$

Therefore, $u$ is called the generalized displacement field since it coincides with the displacement field in the absence of dislocations, i.e., for $\epsilon^{0}=\operatorname{inc} F=0$. Moreover, we set $v:=\frac{d u}{d t}$, the pointwise velocity.

The right-hand side of (3-3) depends on $F$, i.e., through $\operatorname{tr} \epsilon^{0}$, for which an equation must be found. To this end, we appeal to Kröner's relation, proved in [Van Goethem 2016b] and which reads inc $\epsilon=\operatorname{inc} \epsilon^{0}=\operatorname{Curl} \kappa_{\mathcal{L}}$, where the righthand side is a concentrated first-order distribution. However, in the present work, which deals with thermodynamic consideration, the right-hand side will be regularized by convolution with a certain divergence-free mollifier $\eta_{\rho}$ (this amounts to considering a tubular neighborhood of the line of some fixed radius $\rho$, which is a common practice in the dislocation literature). Thus, by (3-2),

$$
\left\{\begin{aligned}
\operatorname{incinc} F & =\mathbb{G}_{\rho}:=\operatorname{Curl} \kappa_{\mathcal{L}} \star \eta_{\rho} & & \text { in } \Omega, \\
F & =0 & & \text { on } \partial \Omega, \\
\left(\partial_{N} F \times N\right)^{t} \times N & =0 & & \text { on } \partial \Omega,
\end{aligned}\right.
$$

where the boundary conditions are chosen in such a way that (3-4) has a unique solution. Indeed, (3-3) and (3-4) are well-posed as discussed in [Van Goethem 2015]. Note that well-posedness in weak form is a direct consequence of coercivity as proved in Section 2. Other boundary conditions of Neumann or mixed type will

${ }^{3}$ Componentwise, $\left(\mathbb{\square}_{4}\right)_{i j k l}=\frac{1}{2}\left(\delta_{i k} \delta_{j l}+\delta_{i l} \delta_{j k}\right)$ and $\left(\mathbb{Q}_{2}\right)_{i j}=\delta_{i j}$. 
be discussed below. Furthermore, $\operatorname{div} \mathbb{G}_{\rho}=0$, and hence, there exists $\kappa$ called the regularized contortion such that

$$
\mathbb{G}_{\rho}=\operatorname{Curl} \kappa .
$$

Note that such a model is also discussed in [Van Goethem 2016a].

\subsection{Chosen boundary conditions.}

Boundary conditions for the gauge field F. The boundary conditions of (3-4) are of essential type (i.e., Dirichlet-like). Note that the first boundary condition on $F$ in (3-4) is required to satisfy the boundary conditions of Beltrami decomposition (3-2) of Theorem 1. Furthermore, it has been shown in Lemma 8 that the second boundary condition for $F$ implies that $\operatorname{Curl}^{t} F \times N=0$ on $\partial \Omega$, which in turn implies that $($ inc $F) N=0$ by Lemma 9 .

On the other hand, in order to determine the natural boundary conditions, a Green formula has been computed in Section 2.1. In particular, the formula shows that the second boundary condition on $F$ may be replaced by a condition on the tangential components of inc $F$. Specifically, the following equation with pure Neumann boundary conditions has a solution [Amstutz and Van Goethem 2016]:

$$
\left\{\begin{aligned}
\operatorname{inc}(\mathbb{M} \text { inc } F) & =\operatorname{Curl} \kappa & & \text { in } \Omega, \\
\mathcal{T}_{0}(\operatorname{inc} F) & =0 & & \text { on } \partial \Omega, \\
\mathcal{T}_{1}(\operatorname{inc} F) & =0 & & \text { on } \partial \Omega,
\end{aligned}\right.
$$

with $\mathbb{M}$ positive-definite and where $\mathcal{T}_{0}$ and $\mathcal{T}_{1}$ are the trace operators as defined in Lemma 4. Note that $\mathcal{T}_{0}(A):=(A \times N)^{t} \times N$ stands by (2-2) for the tangential components of tensor $A$ (in a different order). So we will write

$$
A_{T}:=\mathcal{T}_{0}(A),
$$

with subscript $T$ standing for tangential. To be precise, as a consequence of the Green formula, $\mathcal{T}_{0}$ (inc $\left.F\right)$ is the dual of $\left(\partial_{N} F\right)_{T}$ and $\mathcal{T}_{1}$ (inc $\left.F\right)$ is the dual of $F$. This and the above remark imply that either (inc $F$ ) $N$ or (inc $F)_{T}$ might be prescribed on the boundary but not both simultaneously.

Remark 14. Because (3-6) is given with Neumann boundary conditions, uniqueness might only hold in a quotient space. Specifically, term $\int_{\partial \Omega} \operatorname{Curl}^{t} F \times N d S(x)$ might not vanish in the right-hand side of the coercivity inequality. Hence, $F$ is fixed up to a gauge field $\widetilde{F}$ satisfying $\int_{\partial \Omega} \operatorname{Curl}^{t} \widetilde{F} \times N d S(x)=0$. This kind of detail is not of interest for the purpose of this work, but the curious reader may refer to [Amstutz and Van Goethem 2016].

With a view of the time-evolution model, we would like to justify the chosen boundary condition for $\epsilon^{0}:=\operatorname{inc} F$ as derived in the next section. To this end, 
we must find a set of mixed essential/natural boundary conditions on $F$ and its derivatives that imply $\mathcal{T}_{1}($ inc $F)=0$. First let us make a general remark. There are six unknowns for a fourth-order operator, and hence, twelve complementary conditions must be prescribed on the boundary (for the complete theory, we refer to [Agmon et al. 1964]). This is the case if the symmetric $F$ and $\left(\partial_{N} F\right)_{T}$ are set to zero, for instance, as for the homogeneous Dirichlet boundary condition. For the pure Neumann case, $\mathcal{T}_{0}$ provides three independent conditions and $\mathcal{T}_{1}$ six, whereby there exist three degrees of freedom unprescribed (whence the quotient space).

We assume that $\mathcal{T}_{0}(\operatorname{inc} F$ ) and only the normal components $F N$ are vanishing on the boundary. Then, referring to the Green-formula expression (2-7) and (2-9) with $T=\epsilon^{0}=$ inc $F$, we observe that the central term in the right-hand side of $\mathcal{T}_{1}\left(\epsilon^{0}\right)$ simplifies to $\mathcal{T}_{0}\left(\partial_{N} \epsilon^{0}\right)$. By (2-9) and (2-10), it remains to consider the term $\left(\left(\mathrm{Curl}^{t} \epsilon^{0}\right) \times N\right)^{S}$ and the first term on the right of (2-10). On the one hand, the term $\left(\operatorname{Curl}^{t} \epsilon^{0}\right) \times N$ is related to the dislocation rotation gradient since one recognizes $\mathrm{Curl}^{t} \epsilon$ as the Frank tensor, satisfying for a general strain $\epsilon$ (by Mitchell-CesaroVolterra decomposition and path integrations; see, e.g., [Maggiani et al. 2015])

$$
\nabla \omega=\operatorname{Curl}^{t} \epsilon,
$$

where $\omega$ is the rotation field. Thus, defining the divergence-free dislocation-induced rotation $\omega^{0}$ by means of $\nabla \omega^{0}:=\operatorname{Curl}^{t} \epsilon^{0}$, if we impose that $\omega^{0}$ be constant on $\partial \Omega$, then $\nabla \omega^{0} \times N=\operatorname{Curl}^{t} \epsilon^{0} \times N=0$ on $\partial \Omega$. This is interpreted as a condition of rigid dislocation-induced rotation of the crystal boundary.

Summarizing, by recalling (2-13), if one assumes $F N=0$ (three conditions), $\mathcal{T}_{0}($ inc $F)=\mathcal{T}_{0}\left(\partial_{N}\right.$ inc $\left.F\right)=0(3+3=6$ conditions $)$, and $\omega^{0}=$ constant on $\partial \Omega$ (two conditions), then the second Neumann boundary condition will be zero for a nonflat boundary if we also assume the additional condition $\left(\epsilon^{0}\right)_{N N}:=\epsilon^{0} N \cdot N=0$ (the twelfth and last condition). Note that, as a consequence of $\left(\epsilon^{0}\right)_{T}=\left(\epsilon^{0}\right)_{N N}=0$ on $\partial \Omega$, the trace of $\epsilon^{0}$ vanishes, i.e.,

Obviously,

$$
e:=\operatorname{tr} \epsilon^{0}=0 \quad \text { on } \partial \Omega
$$

$$
\partial_{R} e=\partial_{R} \operatorname{tr} \epsilon^{0}=0 \quad \text { for } R=A, B \text { on } \partial \Omega .
$$

As resulting from the above considerations, from now on in this work, the following equation for $F$ will be considered:

$\left\{\begin{aligned} \operatorname{inc}(\operatorname{inc} F) & =\operatorname{Curl} \kappa & & \text { in } \Omega, \\ F N & =0 & & \text { on } \partial \Omega, \\ \mathcal{T}_{0}(\operatorname{inc} F) & =0 & & \text { on } \partial \Omega, \\ (\operatorname{inc} F) N \cdot N & =0 & & \text { on } \partial \Omega, \\ \mathcal{T}_{0}\left(\partial_{N} \operatorname{inc} F\right) & =0 & & \text { on } \partial \Omega, \\ \operatorname{Curl}^{t}(\operatorname{inc} F) \times N & =0 & & \text { on } \partial \Omega .\end{aligned}\right.$


Furthermore, by elliptic regularity ${ }^{4}$ and the smoothness of $\kappa$, the fields $F$ and inc $F$ are also smooth.

Note that the third line of (3-10) implies that

$$
\epsilon^{0} \times N=-\epsilon_{A A}^{0} \tau_{A} \otimes \tau_{B}+\epsilon_{B B}^{0} \tau_{B} \otimes \tau_{A}+\epsilon_{A B}^{0}\left(\tau_{A} \otimes \tau_{A}-\tau_{B} \otimes \tau_{B}\right)=0 .
$$

Boundary condition for the dislocation strain $\epsilon^{0}$. We recall the following facts. The elastic strain can be written as $\epsilon=\mathbb{A}^{-1} \sigma=\nabla^{S} u+\epsilon^{0}$, where inc $\epsilon=$ inc $\epsilon^{0}=$ $\operatorname{Curl} \kappa$. The tensor $\epsilon^{0}=\operatorname{inc} F$ is called the dislocation strain since it is the only part of the elastic strain related to the dislocation density.

First note that the Neumann conditions $\mathcal{T}_{0}(\operatorname{inc} F)=0$ and $(\operatorname{inc} F) N \cdot N=0$ in (3-10) exactly mean that $\left(\epsilon_{0}\right)_{T}=0$ and $\left(\epsilon_{0}\right)_{N N}=0$, respectively. Thus, they naturally impose Dirichlet boundary conditions for $\epsilon^{0}$ though incomplete since the components $\left(\epsilon_{0} N\right) \cdot \tau^{R}$ remain unprescribed so far (with $\tau^{R}$ the $R$-th tangent vector to $\partial \Omega$ ). We also impose $\left(\operatorname{Curl}^{t} \epsilon^{0}\right) \times N=0$ in (3-10).

In order to chose the remaining boundary conditions for $\epsilon^{0}$, we will require that the following integration by parts be valid:

$$
\mathbb{M i n c} \epsilon^{0} \mid \text { inc } \epsilon^{0}=\operatorname{inc}\left(\mathbb{M} \text { inc } \epsilon^{0}\right) \mid \epsilon^{0} \text {. }
$$

As a consequence of (3-10), we already know that $\left(\epsilon^{0}\right)_{T}=\left(\partial_{N} \epsilon^{0}\right)_{T}=\left(\epsilon_{0}\right)_{N N}=0$ on $\partial \Omega$. Therefore, recalling that $\mathcal{T}_{0}\left(\mathbb{M}\right.$ inc $\left.\epsilon^{0}\right) \cdot \partial_{N} \epsilon^{0}=\left(\mathbb{M}\right.$ inc $\left.\epsilon^{0}\right) \cdot\left(\partial_{N} \epsilon^{0}\right)_{T}=0$ on $\partial \Omega$, in order for (3-12) to hold, it suffices to impose, by referring to Green formula (2-7) with $T=\mathbb{M}$ inc $\epsilon^{0}$, that the boundary integrand $\mathcal{T}_{1}\left(\mathbb{M}\right.$ inc $\left.\epsilon^{0}\right) \cdot \epsilon^{0}=0$ on $\partial \Omega$. Furthermore, by (2-14), only the $N R$-components (for $R=A, B)$ of $\mathcal{T}_{1}\left(\mathbb{M}\right.$ inc $\epsilon^{0}$ ) matter in this product since $\left(\epsilon^{0}\right)_{T}=\left(\epsilon_{0}\right)_{N N}=0$ on $\partial \Omega$. Then only the first and last terms of (2-14) are nonvanishing, and (2-14) is equivalently rewritten by virtue of $(2-3)-(2-5)$ as

$$
\kappa^{R^{*}}\left(\mathbb{M} \text { inc } \epsilon^{0}\right)_{R N}+\partial_{R}\left(\mathbb{M i n c} \epsilon^{0}\right)_{R^{*} R^{*}}-\partial_{R^{*}}\left(\mathbb{M} \text { inc } \epsilon^{0}\right)_{R R^{*},} \quad R=A, B,
$$

where $\tau_{R}$ stands for the derivative along the $R$-th tangent vector $\tau^{R}$ and $\kappa^{R}$ for the $R$-th principal curvature, for $R=A$ or $B$, and with $A^{*}=B$ and $B^{*}=A$. Note that, for a cylindrical boundary, the last two terms are recognized as a surface curl.

Let us remark that, by Lemma 9 , (inc $\left.\epsilon^{0}\right) N=0$ since $\left(\operatorname{Curl}^{t} \epsilon^{0}\right) \times N=0$ on $\partial \Omega$, and hence, if one assumes that $\mathbb{M}$ has the same symmetry as the isotropic elasticity tensor, then $\left(\mathbb{M} \text { inc } \epsilon^{0}\right)_{R N}=0$ and the first term in (3-13) vanishes. In this case, the boundary condition reduces to imposing a vanishing surface curl of $\mathbb{M} \epsilon^{0}$.

${ }^{4}$ The operator inc inc is $\Delta^{2}$ for symmetric solenoidal fields, and equation well-posedness is shown in [Van Goethem 2015]. See also Section 2. 
Summarizing, the following boundary conditions will be prescribed for $\epsilon^{0}$ :

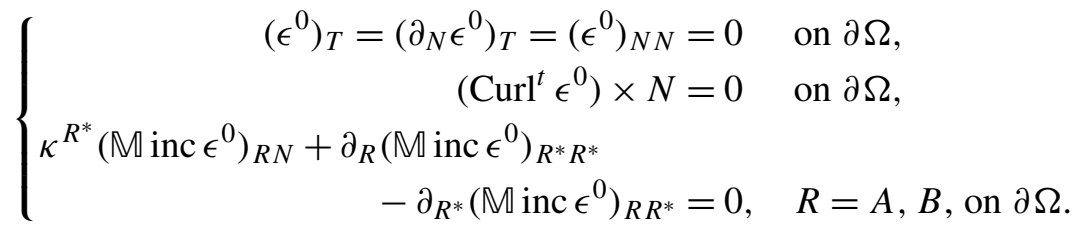

Let us recall that the Dirichlet conditions (i.e., the first two lines in (3-14)) follow from the chosen Neumann conditions for $F$, whereas the Neumann conditions are chosen so as to permit the integration by parts (3-12). As for their physical meaning, $\operatorname{Curl}^{t} \epsilon^{0}$ is the dislocation Frank tensor, i.e., the rotation gradient generated by the dislocations. Moreover, $\mathbb{M}$ inc $\epsilon^{0}=\mathbb{M} \operatorname{Curl} \kappa$ is a dislocation flux as related to the density of dislocation gradients and the crystal symmetries (given by the symmetries of tensor $\mathrm{M}$ ) and material properties. The Neumann condition is satisfied if for instance the dislocation density on the boundary is prescribed such that Curl $\kappa$ is purely tangential and constant on the boundary.

\section{Evolution law for the dislocation strain}

The aim of this section is to derive an evolution law for $\epsilon^{0}$ from the second principle of thermodynamics and by assuming that the evolution of the dislocation density (i.e., $\Lambda$ and hence $\kappa$ ) is known (by means of transport-reaction-diffusion $\mathrm{PDEs}^{5}$ ). To be precise, the model will be derived from a particular form of the global Clausius-Duhem inequality. Let us stress that the obtained evolution law is too simple to satisfy the principle in its full generality. In fact, our aim here is to derive a simple model based on the principle, study its mathematical wellposedness, and leave more elaborate models for future works. In this respect, our aim is also to show that the incompatibility operator naturally appears in the model as soon as high-order dislocation density terms are considered in the free energy. Note that evolution laws are often postulated from the statics equations, but this procedure is questionable since the resulting dynamics does not necessarily comply with thermodynamic principles.

\subsection{Model assumptions.}

Assumptions on the free energy. Let the Helmholtz free energy be given by

$$
\Psi:=\widehat{\Psi}(\epsilon, \kappa, \operatorname{Curl} \kappa)=\widehat{\Psi}_{e}(\epsilon)+\Psi_{\text {dislo }}\left(\epsilon^{0}, \kappa, \operatorname{Curl} \kappa\right),
$$

where a quadratic law in $\kappa$ and $\mathrm{Curl} \kappa$ is postulated:

$$
\Psi_{\text {dislo }}\left(\epsilon^{0}, \kappa, \operatorname{Curl} \kappa\right)=\frac{1}{2} \mathbb{N} \kappa \cdot \kappa+\frac{1}{2} \mathbb{M} \operatorname{Curl} \kappa \cdot \operatorname{Curl} \kappa+\psi_{\text {dislo }}\left(\epsilon^{0}\right)
$$

\footnotetext{
${ }^{5}$ For point defects, such a law was studied in [Van Goethem et al. 2008].
} 
with $\mathbb{M}$ and $\mathbb{N}$ positive-definite fourth-rank tensors. In this work, we will restrict ourselves to symmetric tensors $\mathbb{N}$ of the form

$$
\mathbb{N}=2 \beta \rrbracket_{4},
$$

where $\beta \geq 0$ is a constant scalar and $\left(\rrbracket_{4}\right)_{i j k l}=\frac{1}{2}\left(\delta_{i k} \delta_{j l}+\delta_{i l} \delta_{j k}\right)$. Note that $\mathbb{N}$ and hence $\beta$ have the dimensions of a force since $\kappa$, as $\Lambda$, has the dimensions of an inverse length while $\mathbb{M}$ has the dimensions of a force times a surface.

Let us emphasize that high-order dislocation models involving strain derivatives in the form of $\epsilon$ and its curl are not new; see, e.g., [Berdichevsky 2006].

Assumption of rigid dislocation-induced rotation. We assume that the dislocationinduced rotations are constant along $\partial \Omega$, that is, $\nabla \omega^{0} \times N=\operatorname{Curl}^{t} \epsilon^{0} \times N=0$. Nonetheless, variations of rotation may occur as induced by purely elastic loading since $\operatorname{Curl}^{t} \nabla^{S} u \times N \neq 0$.

Additional remark. The relation inc $\epsilon^{0}=\mathrm{Curl} \kappa$ yields

$$
\kappa=\operatorname{Curl}^{t} \epsilon^{0}+\nabla \varphi
$$

for some vector $\varphi$ satisfying, by the identity $\operatorname{div} \epsilon^{0}=0$,

$$
\mathcal{L}_{0,1}(\varphi)=\operatorname{div} \nabla^{S} \varphi=\operatorname{div} \kappa^{S},
$$

where we have chosen $\varphi=0$ on $\partial \Omega$. Note that this latter choice yields that $\operatorname{Curl}^{t} \epsilon^{0} \times N=0$ on $\partial \Omega$ implies that $\kappa \times N=0$ and hence $\operatorname{Curl} \kappa N=\operatorname{inc} \epsilon N=0$ on $\partial \Omega$, by Lemma 9 .

4.2. Thermodynamics considerations. The notions invoked in this section are classical in thermodynamics. References can be found in, e.g., [Lemaitre and Chaboche 1988; Ottosen and Ristinmaa 2005]. The idea is to derive an evolution law that would at least satisfy the second principle of thermodynamics globally in $\Omega$. The pointwise (otherwise termed local) isothermal Clausius-Duhem inequality reads

$$
\begin{aligned}
0 \leq D & =\sigma \cdot \nabla \dot{u}-\dot{\Psi} \\
& =\sigma \cdot(\dot{\epsilon}-\operatorname{inc} \dot{F})-\delta_{\epsilon} \Psi \cdot \dot{\epsilon}-\delta_{\kappa} \Psi \cdot \dot{\kappa}-\delta_{\operatorname{Curl} \kappa} \Psi \cdot \operatorname{Curl} \dot{\kappa}-\delta_{\epsilon} \Psi \cdot \dot{\epsilon}^{0} \\
& =-\sigma \cdot \operatorname{inc} \dot{F}+\dot{\epsilon}\left(\sigma-\delta_{\epsilon} \widehat{\Psi}_{e}\right)-\delta_{\kappa} \Psi_{\text {dislo }} \cdot \dot{\kappa}-\delta_{\operatorname{Curl} \kappa} \Psi_{\text {dislo }} \cdot \operatorname{Curl} \dot{\kappa}-\delta_{\epsilon^{0}} \psi_{\text {dislo }} \cdot \dot{\epsilon}^{0}
\end{aligned}
$$

(the $\cdot$ symbol stands for the time derivative, i.e., $\dot{\epsilon}:=\partial_{t} \epsilon(x, t)$, and $\nabla \dot{u}$ for the gradient of the velocity field). It is classically deduced that $\sigma=\delta_{\epsilon} \widehat{\Psi}_{e}$ and hence

$$
0 \leq D=-\sigma \cdot \operatorname{inc} \dot{F}-(\mathbb{N} \kappa \cdot \dot{\kappa}+\mathbb{M} \operatorname{Curl} \kappa \cdot \operatorname{Curl} \dot{\kappa})-\delta_{\epsilon^{0}} \psi_{\text {dislo }} \cdot \dot{\epsilon}^{0} .
$$

Introduce the global mechanical dissipation as

$$
\mathscr{D}:=\int_{\Omega} D d x
$$


The isothermal global form of the second law of thermodynamics (or the global Clausius-Duhem inequality) in $\Omega$ reads $^{6}$

$$
\mathscr{D} \geq 0 .
$$

Inequality (4-7) will allow us to derive the sought evolution equation for the dislocation strain. Recall the notation

$$
a \mid b:=\int_{\Omega} a \cdot b d x .
$$

By the symmetry of $\sigma$, Theorem 1 yields a unique $(\psi, \mathbb{S})$ satisfying $\sigma=\nabla^{S} \psi+$ inc $\mathbb{S}$ with $\psi=0, \mathbb{S}=0$, and $\mathrm{Curl}^{t} \mathbb{S} \times N=0$ on $\partial \Omega$ (the same remark as for (3-4) holds for $\mathbb{S}$ ). In particular, one has inc inc $\mathbb{S}=\operatorname{inc} \sigma$, where we remark that the dependence of $\mathbb{S}$ upon $\epsilon^{0}$ must not be linear. Furthermore,

$$
\sigma \cdot \operatorname{inc} \dot{F}=\nabla^{S} \psi \cdot \text { inc } \dot{F}+\text { inc } S \cdot \text { inc } \dot{F},
$$

which by integration by parts (justified by Lemma 8) yields

$$
\sigma \mid \text { inc } \dot{F}=\operatorname{inc} \mathbb{S} \mid \text { inc } \dot{F}=\mathbb{S} \mid \text { inc inc } \dot{F}=\mathbb{S} \mid \text { inc } \dot{\epsilon}=\mathbb{S} \mid \text { inc } \dot{\epsilon}^{0}=\text { inc } \mathbb{S} \mid \dot{\epsilon}^{0} \text {. }
$$

Moreover, by Beltrami decomposition again, the symmetric tensor $\delta_{\epsilon^{0}} \psi_{\text {dislo }}$ can be decomposed as

$$
\delta_{\epsilon^{0}} \psi_{\text {dislo }}=\nabla^{S} \eta+\text { inc } \mathbb{K}_{\epsilon^{0}}
$$

for some vector-valued $\eta$ (here taken with $\eta=0$ on $\partial \Omega$ ), where $\mathbb{K}_{\epsilon^{0}}$ is a symmetric divergence-free tensor whose dependence upon $\epsilon^{0}$ must not be linear too. Hence, recalling the solenoidal property of $\epsilon^{0}, \delta_{\epsilon^{0}} \psi_{\text {dislo }} \mid \dot{\epsilon}^{0}=$ inc $_{\mathbb{\epsilon}^{0}} \mid \dot{\epsilon}^{0}$. Thus, by (4-4), (4-6) and (4-7) can be rewritten as

$$
\begin{aligned}
0 \leq \mathscr{D}=-\operatorname{inc} \mathbb{S}_{\epsilon^{0}} \mid \dot{\epsilon}^{0}-\left(\mathbb { N } \left(\operatorname{Curl}^{t} \epsilon^{0}\right.\right. & +\nabla \varphi) \mid\left(\operatorname{Curl}^{t} \dot{\epsilon}^{0}+\nabla \dot{\varphi}\right) \\
& \left.+\mathbb{M} \text { inc } \epsilon^{0}\left|\operatorname{inc} \dot{\epsilon}^{0}+\operatorname{inc} \mathbb{K}_{\epsilon^{0}}\right| \dot{\epsilon}^{0}\right),
\end{aligned}
$$

where the dependence of $S$ upon $\epsilon^{0}$ has been emphasized by the subscript.

Let us now consider the second term of the right-hand side. By the symmetry property of $\mathbb{N}$ and since $\varphi=\dot{\varphi}=0$ on the boundary,

$$
\mathbb{N} \operatorname{Curl}^{t} \epsilon^{0}\left|\left(\operatorname{Curl}^{t} \dot{\epsilon}^{0}+\nabla \dot{\varphi}\right)=\mathbb{N} \operatorname{Curl}^{t} \epsilon^{0}\right| \operatorname{Curl} \dot{\epsilon}^{0}-\operatorname{div}\left(\mathbb{N} \operatorname{Curl}^{t} \epsilon^{0}\right) \mid \dot{\varphi} .
$$

Obviously, $\operatorname{div} \operatorname{Curl}^{t} \epsilon^{0}=\operatorname{div} \operatorname{Curl} \epsilon^{0}=0$, and hence, integrating $\mathbb{N} \operatorname{Curl}^{t} \epsilon^{0} \mid \operatorname{Curl} \dot{\epsilon}^{0}$ by parts by recalling (4-3) and Lemma 3 allows one to rewrite (4-12) as

$$
(4-12)=\beta \text { inc } \epsilon^{0}\left|\dot{\epsilon}^{0}+\beta \operatorname{Curl} \epsilon^{0}\right| \operatorname{Curl} \dot{\epsilon}^{0}+2 \beta \int_{\partial \Omega}\left(\operatorname{Curl}^{t} \epsilon^{0}\right)^{S} \times N \cdot \dot{\epsilon}^{0} d S,
$$

${ }^{6}$ The global form expressed in full generality would require a positive integral in any timedependent control volume in $\Omega$. 
where the integrand in the boundary term can be rewritten as $-\left(\operatorname{Curl}^{t} \epsilon^{0}\right)^{S} \cdot\left(\dot{\epsilon}^{0} \times N\right)$ and hence vanishes by (3-11). Now, by definition of the Frank tensor (3-7),

$$
\beta \operatorname{Curl} \epsilon^{0}\left|\operatorname{Curl} \dot{\epsilon}^{0}=\beta \operatorname{Curl}^{t} \epsilon^{0}\right| \operatorname{Curl}^{t} \dot{\epsilon}^{0}=\beta \nabla \omega^{0} \mid \nabla \dot{\omega}^{0} .
$$

Therefore, (4-13) can be rewritten as

$$
\beta \text { inc } \epsilon^{0}\left|\dot{\epsilon}^{0}+\beta \nabla \omega^{0}\right| \nabla \dot{\omega}^{0} .
$$

From the right-hand side of (4-11) and by $\operatorname{div} \operatorname{Curl}^{t} \epsilon^{0}=\operatorname{div} \operatorname{Curl} \epsilon^{0}=0$, one is left with

$$
\mathbb{N} \nabla \varphi\left|\left(\operatorname{Curl}^{t} \dot{\epsilon}^{0}+\nabla \dot{\varphi}\right)=\mathbb{N} \nabla^{S} \varphi\right| \nabla^{S} \dot{\varphi}=2 \beta \nabla^{S} \varphi \mid \nabla^{S} \dot{\varphi}
$$

with $\varphi$ the unique solution to (4-5). ${ }^{7}$

Summarizing, (4-11) is rewritten as

$$
\begin{aligned}
0 \leq \mathscr{D} & =-\beta \nabla \omega^{0}\left|\nabla \dot{\omega}^{0}-2 \beta \nabla^{S} \varphi\right| \nabla^{S} \dot{\varphi}-\beta \operatorname{inc} \epsilon^{0}\left|\dot{\epsilon}^{0}-\operatorname{inc}\left(\mathbb{M} \operatorname{inc} \epsilon^{0}+\mathbb{H}_{\epsilon^{0}}\right)\right| \dot{\epsilon}^{0} \\
& =-\frac{d}{d t} \mathscr{E}_{\beta}\left(\epsilon^{0}, \kappa\right)-\operatorname{inc}\left(\mathbb{M i n c} \epsilon^{0}+\beta \epsilon^{0}+\mathbb{H}_{\epsilon^{0}}\right) \mid \dot{\epsilon}^{0}
\end{aligned}
$$

where the nonlinear term with respect to $\epsilon^{0}$ is the symmetric and solenoidal tensor

$$
\mathbb{W}_{\epsilon^{0}}:=\mathbb{K}_{\epsilon^{0}}+\mathbb{S}_{\epsilon^{0}}
$$

and with a stored quadratic dislocation energy $\mathscr{E}_{\beta}$ defined as

$$
\mathscr{E}_{\beta}\left(\epsilon^{0}, \kappa\right):=\frac{\beta}{2}\left(\nabla \omega^{0}\left|\nabla \omega^{0}+2 \nabla^{S} \varphi\right| \nabla^{S} \varphi\right) .
$$

Let us remark that, if the free energy is independent of $\kappa$, that is, if $\beta=0$, then $\mathscr{E}_{\beta}=0$ and (4-16) immediately yields

$$
-\operatorname{inc}\left(\mathbb{M i n c} \epsilon^{0}+\beta \epsilon^{0}+\mathbb{W}_{\epsilon^{0}}\right) \mid \dot{\epsilon}^{0}=\mathscr{D} \geq 0 .
$$

4.3. Time-evolution of the dislocation strain. Let us now consider a certain time scale, which is lower than that of dissipative phenomena associated with the evolution of dislocations (the law for $\kappa$ ) but high enough not to invalidate the hypothesis of local state [Lemaitre and Chaboche 1988]. We will consider a thought experiment with a certain number of pure edge dislocations in such a way that $\varphi=0$, whereas the norm of $\nabla \omega^{0}$ can reach arbitrarily high values. Thus, one can render $-\frac{d}{d t} \mathscr{E}(\epsilon, \kappa)$ arbitrarily negative, and in order for the global dissipation $\mathscr{D}$ to remain positive in (4-16), the term inc $\left(\mathbb{M}\right.$ inc $\left.\epsilon^{0}+\beta \epsilon^{0}+\mathbb{W}_{\epsilon^{0}}\right) \mid \dot{\epsilon}^{0}$ must be nonpositive. For this reason, the following evolution law for $\epsilon^{0}$ is postulated:

$$
0=\alpha \dot{\epsilon}^{0}(t)+\operatorname{inc}\left(\mathbb{M} \text { inc } \epsilon^{0}(t)+\mathcal{G}\left(\epsilon^{0}(t)\right)\right)
$$

${ }^{7}$ Thus, it linearly depends on $\operatorname{div} \kappa^{S}$. 
for some material-dependent coefficient $\alpha \geq 0$ and with the solenoidal tensor-valued nonlinear term

$$
\mathscr{G}\left(\epsilon^{0}\right):=\mathbb{H}_{\epsilon^{0}}+\beta \epsilon^{0}=\mathcal{G}\left(\epsilon^{0}\right)-\mathbb{L},
$$

where $\mathbb{L}$ stands for a symmetric (not necessarily divergence-free) tensor independent of $\epsilon^{0}$. We introduce the generalized dislocation force as the symmetric and solenoidal tensor

$$
\mathbb{G}:=\text { inc } \mathbb{L} \text {. }
$$

Moreover, the boundary conditions (4-19) and the initial condition $\epsilon^{0}(0)=\epsilon_{0}^{0}$ at $t=0$ are prescribed.

Specifically, the sought time-dependent boundary-value problem for the dislocation strain reads, by recalling (3-14),

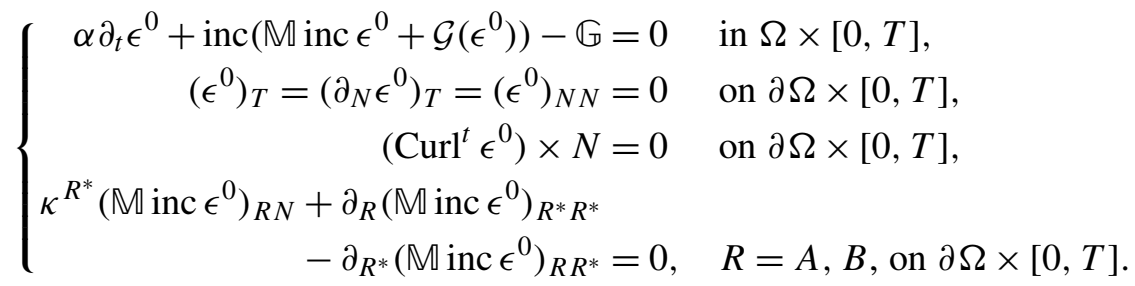

Furthermore, the following energy relation also holds:

$$
\frac{d}{d t} \mathscr{E}_{\beta}(\epsilon, \kappa) \leq \alpha\left|\dot{\epsilon}^{0}\right|^{2}
$$

In particular, the energy $\mathscr{E}_{\beta}$ decreases in time as soon as the dislocation strain is stationary.

\section{Well-posedness of the evolution}

5.1. Weak forms. Recall first the notation $a \mid b=(a, b)_{2}$, where the right-hand side stands for the scalar product in $L^{2}$ (of scalars, vectors, tensors, etc.). The weak form associated with (4-19) reads: for all $t \in[0, T]$, find $E(t) \in \mathcal{H}_{0}(\Omega)$ such that

$\alpha \frac{d E}{d t}(t) \mid F+\mathbb{M}$ inc $E(t)|\operatorname{inc} F+\mathcal{G}(E(t))| \operatorname{inc} F-\mathbb{G} \mid F=0$

$$
\text { for all } F \in \mathcal{H}_{0}(\Omega) \text {, }
$$

where $\mathbb{M}$ is a fourth-rank symmetric and positive-definite tensor, $\mathcal{G}$ is a symmetric tensor-valued nonlinear term (not necessarily divergence-free), $\mathbb{G}$ represents a tensor-valued generalized force, and $\alpha>0$, and such that

$$
E(0)=E_{0} \in L^{2}\left(\Omega ; \mathbb{S}^{3}\right) .
$$

\footnotetext{
${ }^{8}$ The dot and the partial time derivative symbols are equivalent. Recall that, in linearized elasticity, material and partial time derivatives do coincide.
} 
By integration by parts, and recalling Lemma 3, (5-1) can be rewritten as: find $E \in \mathcal{H}_{0}(\Omega)$ such that

$$
\begin{array}{r}
\alpha \frac{d E}{d t} \mid F+\mathbb{M} \text { inc } E|\operatorname{inc} F+\operatorname{Curl} \mathcal{G}(E)| \operatorname{Curl}^{t} F-\mathbb{G} \mid F=0 \\
\text { for all } F \in \mathcal{H}_{0}(\Omega) .
\end{array}
$$

The bilinear form associated with the linear part of the PDE reads

$$
a(E, F)=\mathbb{M} \text { inc } E \mid \text { inc } F \text {. }
$$

Its coercivity in $H^{2}(\Omega)$ is an immediate consequence of Theorem 13 .

Note that in the case of the dislocation model of Section 4.3, $\mathcal{G}\left(\epsilon^{0}\right)=\mathbb{H}_{\epsilon^{0}}+\beta \epsilon^{0}$. Recalling (4-18) and assuming for simplicity that $\mathbb{U}_{\epsilon^{0}}=\mathbb{U}(x)$ is independent of $\epsilon^{0}$, the weak form associated to this linear model can be written as: find $E \in \mathcal{H}_{0}(\Omega)$ such that

$$
\alpha \frac{d E}{d t} \mid F+(\mathbb{M} \text { inc } E+\beta E) \mid \text { inc } F-\widetilde{\mathbb{G}} \mid F=0 \quad \text { for all } F \in \mathcal{H}_{0}(\Omega)
$$

with $\widetilde{\mathbb{G}}:=\mathbb{G}+$ inc $\mathbb{T}$. In this case, the equation is a linearization of the general Cahn-Hilliard system.

Now, if $\mathcal{G}$ is assumed to be an objective tensor, it can be written in terms of its invariants, the first of which is the trace of $E$.

Assumption on the nonlinearity. The nonlinear term is assumed to be written as a polynomial in the trace of $E$ plus an affine term in $E$.

Assumption 15 (nonlinear term). Let $E \in \mathbb{S}^{3}$. It is assumed that

$$
\mathcal{G}(E)=\beta E-\frac{1}{3} \varphi(\operatorname{tr} E) \rrbracket_{2}
$$

with $\beta>0$ a constant scalar and $\varphi$ a scalar-valued polynomial defined as

$$
\varphi(v)=\sum_{i=1}^{2 p-1} \rho_{i} v^{i}, \quad p \geq 2,
$$

where $\rho_{2 p-1}>0$. In particular, $\mathcal{G}(E)$ is a symmetric second-rank tensor.

Remark 16. The divergence of the nonlinear term in (5-5) must not be zero since $\operatorname{div} \mathcal{G}(E)=-\frac{1}{3} \varphi^{\prime}(e) \nabla e \neq 0$ unless $\varphi$ is trivially independent of $e$. However, referring to the dislocation model of Section 4.3, $\operatorname{div} \mathscr{G}(E)=\operatorname{div} \mathcal{G}(E)-\operatorname{div} \mathbb{L}=0$ and hence $\operatorname{div} \mathbb{L}=\frac{1}{3} \varphi^{\prime}(e) \nabla e$. Without going into details (see, e.g., [Scala and Van Goethem 2016; Fosdick and Royer-Carfagni 2004]), [L then plays the role of a constraint reaction to ensure the condition $\operatorname{div} \mathscr{G}(E)=0$, and one could take $\mathbb{L}$ of the form $\mathbb{L}=\mathbb{C} \nabla^{S} w$ for a certain elasticity kind of tensor $\mathbb{C}$ and $w$ an associated vector field. 
Furthermore, one has $(\operatorname{Curl} \mathcal{G}(E))_{i j}=\beta(\operatorname{Curl} E)_{i j}-\frac{1}{3} \epsilon_{i j k} \varphi^{\prime}(\operatorname{tr} E) \partial_{k} \operatorname{tr} E$. It follows that

$$
\operatorname{Curl} \mathcal{G}(E)\left|\operatorname{Curl}^{t} E=\beta \operatorname{Curl} E\right| \operatorname{Curl}^{t} E+\varphi^{\prime}(\operatorname{tr} E) \mid(\nabla \operatorname{tr} E)^{2} .
$$

5.2. Energy estimates. For simplicity, the estimates will be done taking $\alpha=1$.

Theorem 17. Under Assumption 15, let $E$ be a solution of (5-1). Then

$$
\frac{d}{d t}\|E(t)\|_{L^{2}}^{2} \leq C\|E(t)\|_{L^{2}}^{2}
$$

for some $C>0$. Moreover,

$$
\|E\|_{L^{\infty}\left(0, T ; L^{2}\right)}+\|E\|_{L^{2}\left(0, T ; H^{2}\right)}^{2}+\left\|\frac{d E}{d t}\right\|_{L^{2}\left(0, T ; H^{-2}\right)} \leq C\left\|E_{0}\right\|_{L^{2}}^{2} .
$$

These estimates also hold for $E$ a solution of (5-4).

Proof. By (5-6), the polynomial $\sum_{i=1}^{2 p-2} \rho_{i} v^{i}$ is bounded from below by a constant. Hence, by (5-7), there exists $\tilde{c} \geq 0$ such that

$$
\operatorname{Curl} \mathcal{G}(E(t)) \mid \operatorname{Curl}^{t} E(t) \geq-\beta\|\operatorname{Curl} E(t)\|_{L^{2}}^{2}-\tilde{c}\|\nabla \operatorname{tr} E(t)\|_{L^{2}}^{2} .
$$

Denoting $\mathcal{C}(E(t)):=\beta\|\operatorname{Curl} E(t)\|_{L^{2}}^{2}+\tilde{c}\|\nabla \operatorname{tr} E(t)\|_{L^{2}}^{2} \geq 0$ and letting $F=E$ in (5-2), one has

$$
\begin{aligned}
& \frac{d}{d t} \frac{1}{2}\|E(t)\|_{L^{2}}^{2}+\mathbb{M} \text { inc } E(t)|\operatorname{inc} E-\mathcal{C}(E(t))-\mathbb{G}| E(t) \\
& \leq \frac{d}{d t} \frac{1}{2}\|E(t)\|_{L^{2}}^{2}+\mathbb{M} \operatorname{inc} E(t)|\operatorname{inc} E(t)+\operatorname{Curl} \mathcal{G}(E(t))| \operatorname{Curl}^{t} E(t)-\mathbb{G} \mid E(t)=0,
\end{aligned}
$$

and hence, there exists $C_{\mathbb{G}}>0$, a constant independent of $E$, such that

$$
\begin{aligned}
\frac{d}{d t} \frac{1}{2}\|E(t)\|_{L^{2}}^{2}+\mathbb{M i n c} E(t) \mid & \operatorname{inc} E(t) \\
& \leq \mathcal{C}(E(t))+\mathbb{G} \mid E(t) \leq C_{\mathbb{G}}\left(\|\nabla E(t)\|_{L^{2}}^{2}+\|E(t)\|_{L^{2}}\right) .
\end{aligned}
$$

The interpolation inequality and general Cauchy inequality [Evans 2010] yield

$$
\begin{aligned}
\frac{d}{d t} \frac{1}{2}\|E(t)\|_{L^{2}}^{2}+\mathbb{M i n c} E(t) \mid \operatorname{inc} E(t) & \leq c\left(\|E(t)\|_{L^{2}}\|E(t)\|_{H^{2}}+\|E(t)\|_{L^{2}}\right) \\
& \leq c \varepsilon\|E(t)\|_{H^{2}}^{2}+\frac{4 \varepsilon+c}{4 \varepsilon}\|E(t)\|_{L^{2}}^{2}
\end{aligned}
$$

for some constant $c:=c_{\mathbb{G}}>0$, a constant independent of $E$. Furthermore, positivedefiniteness and coercivity (see Theorem 13) of $\mathbb{M}$ yield $C_{\mathbb{M}}\|E\|_{H^{2}} \leq \mathbb{M}$ inc $E \mid$ inc $E$ 
for some constant $C_{\mathbb{M}}>0$. Thus, it follows from Theorem 13 and by choosing $\varepsilon$ small enough that

$$
\frac{d}{d t} \frac{1}{2}\|E(t)\|_{L^{2}}^{2} \leq \frac{d}{d t} \frac{1}{2}\|E(t)\|_{L^{2}}^{2}+\bar{C}\|E(t)\|_{H^{2}}^{2} \leq \frac{4 \varepsilon+c}{2 \varepsilon} \frac{1}{2}\|E(t)\|_{L^{2}}^{2}
$$

for some $\bar{C}(t) \geq 0$ (in the sequel, the dependencies of the constants on $\mathbb{G}$ and $\mathbb{M}$ are omitted for conciseness). As a consequence of the differential form of the Gronwall lemma [Evans 2010, §B.2.j] and (5-11), we deduce that

$$
\max _{t \in[0, T]}\|E(t)\|_{L^{2}} \leq C\left\|E_{0}\right\|_{L^{2}}
$$

for some constant $C>0$. Moreover, by (5-10) and time integration in $[0, T]$,

$$
\int_{0}^{t} \frac{d}{d s} \frac{1}{2}\|E(s)\|_{L^{2}}^{2} d s+\bar{C} \int_{0}^{t}\|E(s)\|_{H^{2}}^{2} d s \leq \widehat{C} \int_{0}^{t}\|E(s)\|_{L^{2}}^{2} d s
$$

for some $\widehat{C} \geq 0$. Hence, by (5-12),

$$
\frac{1}{2}\|E(t)\|_{L^{2}}^{2}-\frac{1}{2}\|E(0)\|_{L^{2}}^{2}+\bar{C} \int_{0}^{t}\|E(s)\|_{H^{2}}^{2} d s \leq C \widehat{C} T\left\|E_{0}\right\|_{L^{2}},
$$

and thus,

$$
\|E\|_{L^{2}\left(0, T ; H^{2}\right)}^{2}:=\int_{0}^{T}\|E(t)\|_{H^{2}}^{2} d t \leq \frac{2 \widehat{C} C T+1}{2 \bar{C}}\left\|E_{0}\right\|_{L^{2}}^{2} .
$$

To conclude, take any $V \in H_{0}^{2}\left(\Omega ; \mathbb{M}^{3}\right)$ and let $F=V$ in (5-2).

Set $V=V^{S}+V^{A}$, the symmetric-skewsymmetric decomposition of $V$, and $V^{S}=\nabla^{S} v+V^{0}$, the Beltrami decomposition of its symmetric part, with $V^{0} \in$ $\mathcal{H}_{0}(\Omega)$. Then, by means of some integration by parts,

$$
\begin{aligned}
\frac{d E}{d t}|V+\mathbb{M i n c} E| \text { inc } V & +\mathcal{G}(E) \mid \text { inc } V-\mathbb{G} \mid V \\
& =\frac{d E}{d t} \mid V^{0}+M \text { inc } E \mid \text { inc } V^{0}+\mathcal{G}(E) \mid \text { inc } V^{0}-\mathbb{G} \mid V^{0} \\
& =0 .
\end{aligned}
$$

Thus, we have for some constants $C_{1}, C_{2}^{i}>0$

$$
\left|\frac{d E}{d t}\right| V \mid \leq C_{1}\|E\|_{H^{2}}\|V\|_{H^{2}}+\sum_{i=0}^{2 p-1} C_{2}^{i}\|E\|_{L^{2}}^{i}\|V\|_{H^{2}},
$$

and hence, by (5-12) and (5-15) and with a nonrelabeled constant $C>0$,

$$
\left\|\frac{d E}{d t}\right\|_{L^{2}\left(0, T ; H^{-2}\right)}:=\int_{0}^{T}\left\|\frac{d E}{d t}\right\|_{H^{-2}} d t \leq C\left(\left\|E_{0}\right\|_{L^{2}}^{2}+\sum_{i=0}^{2 p-1}\left\|E_{0}\right\|_{L^{2}}^{i}\right),
$$


where $H^{-2}(\Omega):=\left(H_{0}^{2}\left(\Omega ; \mathbb{M}^{3}\right)\right)^{\prime}$. The proof follows from (5-12), (5-14), and (5-16) since, for the second statement, it suffices to take $\varphi \equiv 0$.

5.3. Existence and uniqueness of the weak solution. It is now well-known that the energy estimates of Theorem 17 and classical decomposition in discrete subspaces of $H^{2}$, the so-called Galerkin approximation (see, e.g., [Evans 2010; Temam 1997; Roubíček 2005]), yield the following theorem. Note that compactness is recovered in $H^{2}\left(\Omega ; \mathbb{S}^{3}\right)$ while the divergence-free properties also pass to the limit. Therefore, the solution belongs to $\mathcal{H}_{0}(\Omega)$ by the second statement of Lemma 8 .

Theorem 18. There exists a unique weak solution $E$ of (5-1) and (5-4) in $\mathcal{H}_{0}(\Omega)$. Moreover, $E \in \mathscr{C}\left(0, T ; H^{-2}\right)$.

Note that continuity in time is an immediate consequence of (5-9).

\section{Discussion}

6.1. Tensor version of Cahn-Hilliard. The derived equations are similar in form to the well-known Cahn-Hilliard equations, but here the variable is a divergencefree tensor $E$. Recall the strong form of (5-1) in $\Omega$ :

$$
\alpha \frac{d E}{d t}(t)+\operatorname{inc}(\mathbb{M} \text { inc } E(t)+\mathscr{G}(E(t)))=0 .
$$

Recall the identity $\operatorname{tr} \operatorname{inc} A=\Delta \operatorname{tr} A-\operatorname{div} \operatorname{div} A$. Then Assumption 15 yields

$$
\operatorname{trinc} \mathscr{G}(E)=\Delta \operatorname{tr}(\mathcal{G}(E)-\mathbb{L})=-\Delta \operatorname{tr} \mathbb{L}+\beta \operatorname{tr} E-\varphi(\operatorname{tr} E)
$$

since $\operatorname{tr}$ inc $A=\Delta \operatorname{tr} A$ for solenoidal fields $A$. Assume also that $\mathbb{M}=2 \tilde{\mu} \rrbracket_{4}+\tilde{\lambda} \rrbracket_{2} \otimes \mathbb{q}_{2}$ for some $\tilde{\mu}>0$, and set $\tilde{\beta}:=2(\tilde{\mu}+\tilde{\lambda})$.

Let us introduce

$$
e:=\operatorname{tr} E
$$

and compute the trace of (6-1). By (6-2),

$$
\begin{aligned}
\alpha e^{\prime}(t) & =\operatorname{trinc}(-\mathbb{M} \operatorname{inc} E(t)-\mathscr{G}(E(t))) \\
& =\Delta(-\tilde{\beta} \Delta e(t)-\beta e(t)+\varphi(e(t))+\operatorname{tr} \mathbb{L}),
\end{aligned}
$$

or more simply,

$$
\alpha e^{\prime}(t)=\Delta(-\tilde{\beta} \Delta e(t)+\psi(e(t))), \quad \psi(e):=\varphi(e)-\beta e+\operatorname{tr} \mathbb{L},
$$

which is recognized as the classical scalar version of the Cahn-Hilliard equation for $e$ with the nonlinear term $\psi$. Note that, in the classical derivation of the CahnHilliard equation, $\tilde{\beta}$ should depend on a small parameter related to a scaling in the free energy. In terms of our model, the part of the strain that is relevant for the variations of dislocation density, i.e., $E=\epsilon^{0}$ (by the relation $\operatorname{Curl} \kappa=\operatorname{inc} \epsilon^{0}$ ), 
has a trace $e$ and therefore is interpreted as dislocation-induced variation of matter density. It is remarkable that $e$ obeys the law (6-4).

Regarding its boundary conditions, it is already known by (3-8) that $e=0$ on $\partial \Omega$. We also assume that $\Delta e=0$ on $\partial \Omega$ and the initial condition $e(0)=\operatorname{tr} E_{0}$. It is wellknown that (6-3) is well-posed (see [Elliott and Stuart 1996] for this particular choice of boundary conditions) though the solution might only be unique up to some gauges since $\partial_{N} e$ is not fixed.

Moreover, $\frac{d}{d t} \int_{\Omega} e d x=-\int_{\partial \Omega} \tilde{\beta} \partial_{N} \Delta e d S(x)+\int_{\partial \Omega}\left(\varphi^{\prime}(e)-\beta\right) \partial_{N} e d S(x)$ clearly follows. From a physical viewpoint, this property simply reflects the inflow of point defects. In fact, any variation of $e$ is due to the change in interstitial and vacancy densities. In some sense, $e$ might be viewed as a point-defect density: positive in the case of an excess of interstitials and negative if vacancies exceed interstitials. Furthermore, assuming that $e$ depends on the temperature $T$, one has a leading boundary inflow proportional to the normal temperature gradient, i.e., given by $\left(\varphi^{\prime}(e)-\beta\right) e^{\prime}(T) \partial_{N} T$. Hence, the point defects will be conserved: $\frac{d}{d t} \int_{\Omega} e d x=0$ as soon as the normal temperature gradient vanishes at the boundary. Otherwise, point defects will be introduced or removed from the boundary. Furthermore, the fact that $e=0$ on $\partial \Omega$ means that point defects are only present inside $\Omega$. Note that point defects on the boundary is somewhat nonsensical since an excess/lack of atoms indeed changes the boundary location. Recall also that dislocations are nucleated by the collapse of point-defect clusters. Hence, determining their density is crucial for dislocation modeling.

Note also that $e$ is the potential yielding the bulk dislocation force $\nabla e$ in (3-3). Therefore, the work done by this force only depends on the variation of point-defect density at the path endpoints. Specifically, the displacement is a solution to

$$
\left\{\begin{aligned}
\rho \partial_{t}^{2} u-\operatorname{div}\left(\mathbb{A} \nabla^{S} u\right) & =f+\lambda \nabla e & & \text { in } \Omega \times[0, T], \\
\left(\mathbb{A} \nabla^{S} u\right) N & =g-\lambda e N & & \text { on } \partial \Omega \times[0, T]
\end{aligned}\right.
$$

as coupled with the point-defect density

$$
\left\{\begin{array}{rlrl}
\partial_{t} e(t)+\Delta(\tilde{\beta} \Delta e(t)+\psi(e(t))) & =0 & & \text { in } \Omega \times[0, T], \\
e=\Delta e=0 & & \text { on } \partial \Omega \times[0, T], \\
e(0) & =e_{0} & & \text { in } \Omega \times[0, T],
\end{array}\right.
$$

where the bulk force term in the right-hand side will be explained in the next subsection. It represents a dissipative force related to point defects as a source or sink.

6.2. Comment about the forcing term. Note that tr $\mathbb{L}$ in (6-3) stands for an external time-dependent field in Gurtin's formalism of microforce balance [1996].

Let us rewrite (4-10) as $\delta_{\epsilon^{0}} \psi_{\text {dislo }}=\mathbb{P}^{C}+\mathbb{P}^{D}$, where the symmetric gradient $\mathbb{P}^{C}$ is impactless on the mechanical dissipation. Accordingly, let $\psi_{\text {dislo }}=\psi_{\text {dislo }}^{C}+\psi_{\text {dislo }}^{D}$, where the first term is a conservative contribution while the latter is dissipative. 
One has $\operatorname{div} \mathbb{P}^{D}=\operatorname{div} \delta_{\epsilon^{0}} \psi_{\text {dislo }}^{D}=\delta_{\epsilon^{0}}^{2} \psi_{\text {dislo }}^{D} \nabla \epsilon^{0}=0$, which implies that $\psi_{\text {dislo }}^{D}$ must be affine in $\epsilon^{0}$. Hence,

$$
\psi_{\text {dislo }}^{D}\left(\epsilon^{0}\right)=\operatorname{inc} \mathbb{K}_{0} \cdot \epsilon^{0}+\mathbb{C}_{0}
$$

for some constant tensor $\mathbb{C}_{0}$. Now, from the expression of the dissipation term of (4-11), inc $\mathbb{K}_{0}\left|\dot{\epsilon}^{0}=\mathbb{K}_{0}\right|$ inc $\dot{\epsilon}^{0}=\mathbb{K}_{0} \mid$ Curl $\dot{\kappa}$, one recognizes $\mathbb{K}_{0}$ as a thermodynamic force.

Now, letting

$$
\psi_{\text {dislo }}^{C}\left(\epsilon^{0}\right)=\psi^{C}\left(\operatorname{tr} \epsilon^{0}\right)+\tilde{\psi}^{C}\left(\nabla \operatorname{tr} \epsilon^{0}\right),
$$

with $\psi^{C}(e)=\int_{0}^{e} \varphi(v) d v-\frac{1}{2} \beta e^{2}+\operatorname{tr} \llbracket e$ and $\tilde{\psi}^{C}(\nabla e)=\frac{1}{2} \tilde{\beta} \nabla e \cdot \nabla e,(6-6)$ can be rewritten as the classical parabolic diffusion equation of the form

$$
\left\{\begin{aligned}
\partial_{t} e(t)+\operatorname{div} j=0 & \text { in } \Omega \times[0, T] \text { with } j:=-\nabla \mu \\
& \text { and } \mu:=\delta_{e}\left(\psi^{C}(e)+\tilde{\psi}^{C}(\nabla e)\right), \\
e=\Delta e=0 & \text { on } \partial \Omega \times[0, T], \\
e(0)=e_{0} & \text { in } \Omega \times[0, T] .
\end{aligned}\right.
$$

6.3. Gradient flow. Let us assume the existence of a scalar $\mathscr{H}$ such that

$$
\text { (inc } \left.\mathscr{G}\left(\epsilon^{0}\right), F\right)=\lim _{\epsilon \rightarrow 0} \frac{\mathscr{H}\left(\epsilon^{0}+\epsilon F\right)-\mathscr{H}\left(\epsilon^{0}\right)}{\epsilon}
$$

for every $F \in \mathcal{H}_{0}(\Omega)$. Note that, for a nonlinear term of the form (5-5), one has $\mathscr{H}\left(\epsilon^{0}\right)=-\left(\mathbb{G}, \epsilon^{0}\right)+\frac{1}{2} \beta\left(\operatorname{Curl}^{t} \epsilon^{0}, \operatorname{Curl} \epsilon^{0}\right)-\frac{1}{3} \phi\left(\operatorname{tr} \epsilon^{0}\right) \rrbracket_{2}$, where $\varphi=\phi^{\prime}$. By defining the incompatibility energy as

$$
\mathscr{E}\left(\epsilon^{0}\right):=\int_{\Omega} \frac{1}{2} \mathbb{M} \text { inc } \epsilon^{0} \cdot \operatorname{inc} \epsilon^{0} d x+\mathscr{H}\left(\epsilon^{0}\right),
$$

it is obvious by integration by parts that the Gâteaux derivative of $\mathscr{E}$ at $\epsilon^{0} \in \mathscr{C}^{\infty}(\Omega)$ in the direction $F \in \mathcal{H}_{0}(\Omega)$ reads $\left\langle\operatorname{grad}_{L^{2}}^{\mathcal{H}_{0}} \mathscr{E}\left(\epsilon^{0}\right), F\right\rangle=\int_{\Omega} \operatorname{inc}\left(\mathbb{M}\right.$ inc $\left.\epsilon^{0}+\mathscr{G}\left(\epsilon^{0}\right)\right) \cdot F d x=\int_{\Omega}\left(\mathbb{M}\right.$ inc $\left.\epsilon^{0}+\mathscr{G}\left(\epsilon^{0}\right)\right) \cdot \operatorname{inc} F d x$. Thus, for a general $\epsilon^{0} \in \mathcal{H}(\Omega)$, one defines the $\mathcal{H}^{-1}$-gradient

$$
\left\langle\operatorname{grad}_{\mathcal{H}^{-1}}^{\mathcal{H}_{0}} \mathscr{E}\left(\epsilon^{0}\right), F\right\rangle:=\int_{\Omega}\left(\mathbb{M} \text { inc } \epsilon^{0}+\mathscr{G}\left(\epsilon^{0}\right)\right) \cdot \operatorname{inc} F d x,
$$

where $\mathcal{H}^{-1}:=\left(\mathcal{H}_{0}(\Omega)\right)^{\prime}$. Due to the Riesz theorem, the linear and continuous map $\operatorname{grad}_{\mathcal{H}^{-1}}^{\mathcal{H}_{0}} \mathscr{E}\left(\epsilon^{0}\right)$ is associated with a unique $F^{0} \in \mathcal{H}_{0}(\Omega)$ such that inc inc $F^{0}=$ $\operatorname{inc}\left(\mathbb{M}\right.$ inc $\left.\epsilon^{0}+\mathscr{G}\left(\epsilon^{0}\right)\right)$, so $\left\langle\operatorname{grad}_{\mathcal{H}^{-1}}^{\mathcal{H}_{0}} \mathscr{E}\left(\epsilon^{0}\right), F\right\rangle:=\int_{\Omega}$ inc $F^{0}$. inc $F d x$. By analogy with the scalar Cahn-Hilliard system, let us call - inc $F^{0}$ the "chemical potential". 
Furthermore, $\left\|\operatorname{grad}_{\mathcal{H}^{-1}}^{\mathcal{H}_{0}} \mathscr{E}\left(\epsilon^{0}\right)\right\|_{\mathcal{H}^{-1}}^{2}=\|$ inc $F^{0} \|_{L^{2}}^{2}=\left(\mathbb{M} \text { inc } \epsilon^{0}+\mathscr{G}\left(\epsilon^{0}\right) \text {, inc } F^{0}\right)_{L^{2}}$, and hence, our model evolution equation can be written as the $\mathcal{H}^{-1}$-gradient flow

$$
\partial_{t} \epsilon^{0}=-\frac{1}{\alpha} \operatorname{grad}_{\mathcal{H}^{-1}}^{\mathcal{H}_{0}} \mathscr{E}:=-\frac{1}{\alpha} \operatorname{inc}\left(\mathbb{M} \operatorname{inc} \epsilon^{0}+\mathscr{G}\left(\epsilon^{0}\right)\right) .
$$

Now, (6-8) implies that

$$
\begin{array}{r}
\frac{d}{d t} \mathscr{E}=\left\langle\operatorname{grad}_{\mathcal{H}^{-1}}^{\mathcal{H}_{0}} \mathscr{E}, \dot{\epsilon}^{0}\right\rangle=\left\langle\operatorname{inc}\left(\mathbb{M i n c} \epsilon^{0}+\mathscr{G}\left(\epsilon^{0}\right)\right), \dot{\epsilon}^{0}\right\rangle=\left(\mathbb{M} \text { inc } \epsilon^{0}+\mathscr{G}\left(\epsilon^{0}\right), \operatorname{inc} \dot{\epsilon}^{0}\right)_{L^{2}} \\
=-\frac{1}{2}\left(\alpha\left|\dot{\epsilon}^{0}\right|^{2}+\frac{1}{\alpha}\left\|\operatorname{grad}_{\mathcal{H}^{-1}}^{\mathcal{H}_{0}} \mathscr{E}\right\|_{\mathcal{H}^{-1}(\Omega)}^{2}\right)=-\frac{1}{\alpha} \| \operatorname{grad}_{\mathcal{H}^{-1}}^{\mathcal{H}_{0}} \mathscr{E}_{\mathcal{H}^{-1}(\Omega)}^{2} \leq 0,
\end{array}
$$

and hence, (4-16) can be written as

$$
0 \leq \mathscr{D}=-\frac{d}{d t}\left(\mathscr{E}_{\beta}\left(\epsilon^{0}, \kappa\right)+\mathscr{E}\left(\epsilon^{0}\right)\right)=\mathscr{D}_{\text {dislo }}+\mathscr{D}_{\text {incomp }}
$$

with the dislocation-induced dissipation term $\mathscr{D}_{\text {dislo }}:=-\frac{d}{d t} \mathscr{E}_{\beta}\left(\epsilon^{0}, \kappa\right)$ vanishing as soon as the free energy is independent of $\kappa$ while the incompatibility-induced dissipation term $\mathscr{D}_{\text {incomp }}:=(1 / \alpha)\left\|\operatorname{grad}_{\mathcal{H}^{-1}}^{\mathcal{H}_{0}} \mathscr{E}\right\|_{\mathcal{H}^{-1}(\Omega)}^{2}=-\frac{d}{d t} \mathscr{E}$ is due to the dependence of the free energy on $\operatorname{Curl} \kappa=$ inc $\epsilon^{0}$. Thus, the incompatibility-induced dissipated energy in $[0, T]$ is decreasing since

$$
\mathscr{E}_{\text {incomp }}:=\int_{0}^{T} \mathscr{D}_{\text {incomp }} d t=\mathscr{E}(0)-\mathscr{E}(T) \geq 0,
$$

and hence, stationarity means that minimization is reached. Therefore, the system should progress toward a stable equilibrium state, a global minimizer of $\mathscr{E}$. This is the basic justification for minimization schemes in a quasistatic setting. Note that maximizing the incompatibility-induced dissipated energy is equivalent to minimizing the incompatibility energy $\mathscr{E}(T)$. Set $\beta=\phi=0$. Then for long-time behavior, one can simply consider the variational problem

$$
\inf _{E \in \mathcal{H}^{\star} \subset \mathcal{H}(\Omega)} \int_{\Omega}\left(\frac{1}{2} \mathbb{M} \text { inc } E \cdot \operatorname{inc} E-\mathbb{G} \cdot E\right) d x
$$

as done in [Amstutz and Van Goethem 2016].

6.4. Concluding remark. This work represents the first step towards a deep understanding of time evolution of dislocation networks at the mesoscale. Its principal aim was to shed light on the importance of the incompatibility operator in the study of dislocations and to propose an evolution in time of the dislocation-induced strain. This required first introducing and/or recalling some properties of this operator as well as its appropriate functional space. The evolution law is based on thermodynamical principles and on the postulate of maximal dissipation adopted for the model internal variables. It turns out that, as a consequence of the second law 
of thermodynamics, the evolution takes the form of the tensor formulation of the Cahn-Hilliard system

$$
\alpha \partial_{t} \epsilon^{0}=\text { inc } \mu,
$$

where $\mu:=-\left(\mathbb{M}\right.$ inc $\left.\epsilon^{0}+\mathscr{G}\left(\epsilon^{0}\right)\right)$ is called the tensor "chemical potential" with $\mathbb{M}$ a positive-definite fourth-rank tensor with the dimensions of a force times a squared distance.

Moreover, the classical scalar Cahn-Hilliard system is recovered for the trace of the dislocation strain, called $e$, which is interpreted as the density of point defects since it allows one to change the solid density by adding or removing single atoms. Remark that this fourth-order equation for $e$ is not classical at all since point defects are classically modeled by second-order reaction-diffusion equations [Van Goethem et al. 2008]. Furthermore, $\nabla e$ also appears to play the role of a conservative bulk force in the displacement equation. Note also that the thermodynamic derivation of the model equations leads to a nonlinear term whose explicit expression is not known. For simplicity, we have considered a general polynomial term in the trace of $\epsilon^{0}$, i.e., in $e$. Of course, more elaborate choices can be made with a view of a general model, but note that the physical sense of the other two invariants of the dislocation strain is not clear.

A crucial quantity we have introduced is the incompatibility energy

$$
\mathscr{E}(E):=\int_{\Omega}\left(\frac{1}{2} \mathbb{M} \text { inc } E \cdot \operatorname{inc} E+\frac{1}{2} \beta \text { inc } E \cdot E-\frac{1}{3} \phi(\operatorname{tr} E)-\mathbb{G} \cdot E\right) d x
$$

with $\beta>0$ a scalar with the dimensions of a force and the scalar $\phi$ and solenoidal tensor $\mathbb{G}$ with the dimensions of a surface force density. Furthermore, $\phi$ is a nonlinear potential depending on $e=\operatorname{tr} E$ and related to point defects. We have shown that the time evolution of the incompatible strain $\epsilon^{0}$ is given by (6-9), which in turn yields the energy equation

$$
\alpha \frac{d}{d t} \mathscr{E}\left(\epsilon^{0}\right)+\left\|\operatorname{grad}_{\mathcal{H}^{-1}}^{\mathcal{H}_{0}} \mathscr{E}\right\|_{\mathcal{H}^{-1}(\Omega)}^{2}=0
$$

for some $\alpha>0$. Therefore, solutions for large times, $\epsilon_{\infty}^{0}$, should approach the minima of the incompatibility energy, namely

$$
\mathscr{E}\left(\epsilon_{\infty}^{0}\right)=\inf _{E \in \mathcal{H}^{\star} \subset \mathcal{H}(\Omega)} \mathscr{E}(E),
$$

which may therefore be considered the associated quasistatic variational problem.

This work and the formalism introduced are expected to clear the way for more involved, complete, and realistic models for the evolution of dislocation networks at the mesoscale. In particular, the interface dynamics in a model of point defects and dislocations in single crystals will be discussed in future work. 


\section{Acknowledgements}

The author was supported by the FCT Starting Grant "Mathematical theory of dislocations: geometry, analysis, and modeling" (IF/00734/2013). The author thanks Samuel Amstutz for fruitful discussions about incompatibility-based models.

\section{References}

[Agmon et al. 1964] S. Agmon, A. Douglis, and L. Nirenberg, "Estimates near the boundary for solutions of elliptic partial differential equations satisfying general boundary conditions, II", Comm. Pure Appl. Math. 17 (1964), 35-92.

[Amstutz and Van Goethem 2016] S. Amstutz and N. Van Goethem, "Analysis of the incompatibility operator and application in intrinsic elasticity with dislocations", SIAM J. Math. Anal. 48:1 (2016), 320-348.

[Berdichevsky 2006] V. L. Berdichevsky, "Continuum theory of dislocations revisited", Contin. Mech. Thermodyn. 18:3-4 (2006), 195-222.

[Bolik and von Wahl 1997] J. Bolik and W. von Wahl, "Estimating $\nabla \boldsymbol{u}$ in terms of div $\boldsymbol{u}, \operatorname{curl} \boldsymbol{u}$, either $(v, \boldsymbol{u})$ or $v \times \boldsymbol{u}$ and the topology", Math. Methods Appl. Sci. 20:9 (1997), 737-744.

[Elliott and Stuart 1996] C. M. Elliott and A. M. Stuart, "Viscous Cahn-Hilliard equation, II: Analysis”, J. Differential Equations 128:2 (1996), 387-414.

[Evans 2010] L. C. Evans, Partial differential equations, 2nd ed., Graduate Studies in Mathematics 19, American Mathematical Society, Providence, RI, 2010.

[Fosdick and Royer-Carfagni 2004] R. Fosdick and G. Royer-Carfagni, "Stress as a constraint reaction in rigid bodies”, J. Elasticity 74:3 (2004), 265-276.

[Gurtin 1996] M. E. Gurtin, "Generalized Ginzburg-Landau and Cahn-Hilliard equations based on a microforce balance”, Phys. D 92:3-4 (1996), 178-192.

[Henrot and Pierre 2005] A. Henrot and M. Pierre, Variation et optimisation de formes: une analyse géométrique, Mathématiques \& Applications 48, Springer, Berlin, 2005.

[Kozono and Yanagisawa 2009] H. Kozono and T. Yanagisawa, " $L^{r}$-variational inequality for vector fields and the Helmholtz-Weyl decomposition in bounded domains", Indiana Univ. Math. J. 58:4 (2009), 1853-1920.

[Lemaitre and Chaboche 1988] J. Lemaitre and J.-L. Chaboche, Mécanique des matériaux solides, 2nd ed., Dunod, Paris, 1988.

[Maggiani et al. 2015] G. B. Maggiani, R. Scala, and N. Van Goethem, "A compatible-incompatible decomposition of symmetric tensors in $L^{p}$ with application to elasticity", Math. Methods Appl. Sci. 38:18 (2015), 5217-5230.

[Maugin 2015] G. A. Maugin, "The saga of internal variables of state in continuum thermo-mechanics (1893-2013)", Mech. Res. Commun. 69 (2015), 79-86.

[Ottosen and Ristinmaa 2005] N. S. Ottosen and M. Ristinmaa, The mechanics of constitutive modeling, Elsevier, Amsterdam, 2005.

[Roubíček 2005] T. Roubíček, Nonlinear partial differential equations with applications, International Series of Numerical Mathematics 153, Birkhäuser, Basel, 2005.

[Scala and Van Goethem 2016] R. Scala and N. Van Goethem, "Constraint reaction and the PeachKoehler force for dislocation networks", preprint, 2016, Available at https://hal.archives-ouvertes.fr/ hal-01213861v2. To appear in Math. Mech. Complex Sys. 
[Temam 1997] R. Temam, Infinite-dimensional dynamical systems in mechanics and physics, 2nd ed., Applied Mathematical Sciences 68, Springer, New York, 1997.

[Van Goethem 2014] N. Van Goethem, "Thermodynamic forces in single crystals with dislocations", Z. Angew. Math. Phys. 65:3 (2014), 549-586.

[Van Goethem 2015] N. Van Goethem, "Cauchy elasticity with dislocations in the small strain assumption”, Appl. Math. Lett. 46 (2015), 94-99.

[Van Goethem 2016a] N. Van Goethem, "Direct expression of incompatibility in curvilinear systems", ANZIAM J. 58:1 (2016), 33-50.

[Van Goethem 2016b] N. Van Goethem, "Incompatibility-governed singularities in linear elasticity with dislocations", Math. Mech. Solids (online publication May 2016).

[Van Goethem et al. 2008] N. Van Goethem, A. de Potter, N. Van den Bogaert, and F. Dupret, "Dynamic prediction of point defects in Czochralski silicon growth: an attempt to reconcile experimental defect diffusion coefficients with the $V / G$ criterion", J. Phys. Chem. Solids 69:2-3 (2008), $320-324$.

[von Wahl 1992] W. von Wahl, "Estimating $\nabla u$ by div $u$ and curl $u$ ", Math. Methods Appl. Sci. 15:2 (1992), 123-143.

Received 25 Jan 2016. Revised 23 May 2016. Accepted 30 Jun 2016.

Nicolas VAn Goethem: vangoeth@fc.ul.pt

Departamento de Matemática, CMAF CIO, Universidade de Lisboa, Alameda da Universidade, C6, 1749-016 Lisboa, Portugal 



\section{Guidelines for Authors}

Authors may submit manuscripts in PDF format on-line at the submission page.

Originality. Submission of a manuscript acknowledges that the manuscript is original and and is not, in whole or in part, published or under consideration for publication elsewhere. It is understood also that the manuscript will not be submitted elsewhere while under consideration for publication in this journal.

Language. Articles in MEMOCS are usually in English, but articles written in other languages are welcome.

Required items. A brief abstract of about 150 words or less must be included. It should be selfcontained and not make any reference to the bibliography. If the article is not in English, two versions of the abstract must be included, one in the language of the article and one in English. Also required are keywords and a Mathematics Subject Classification or a Physics and Astronomy Classification Scheme code for the article, and, for each author, postal address, affiliation (if appropriate), and email address if available. A home-page URL is optional.

Format. Authors are encouraged to use $\mathrm{IAT}_{\mathrm{E} X}$ and the standard amsart class, but submissions in other varieties of $\mathrm{T}_{\mathrm{E}} \mathrm{X}$, and exceptionally in other formats, are acceptable. Initial uploads should normally be in PDF format; after the refereeing process we will ask you to submit all source material.

References. Bibliographical references should be complete, including article titles and page ranges. All references in the bibliography should be cited in the text. The use of $\mathrm{BIBT}_{\mathrm{E}} \mathrm{X}$ is preferred but not required. Tags will be converted to the house format, however, for submission you may use the format of your choice. Links will be provided to all literature with known web locations and authors are encouraged to provide their own links in addition to those supplied in the editorial process.

Figures. Figures must be of publication quality. After acceptance, you will need to submit the original source files in vector graphics format for all diagrams in your manuscript: vector EPS or vector PDF files are the most useful.

Most drawing and graphing packages - Mathematica, Adobe Illustrator, Corel Draw, MATLAB, etc. - allow the user to save files in one of these formats. Make sure that what you are saving is vector graphics and not a bitmap. If you need help, please write to graphics@msp.org with as many details as you can about how your graphics were generated.

Bundle your figure files into a single archive (using zip, tar, rar or other format of your choice) and upload on the link you been provided at acceptance time. Each figure should be captioned and numbered so that it can float. Small figures occupying no more than three lines of vertical space can be kept in the text ("the curve looks like this:"). It is acceptable to submit a manuscript with all figures at the end, if their placement is specified in the text by means of comments such as "Place Figure 1 here". The same considerations apply to tables.

White Space. Forced line breaks or page breaks should not be inserted in the document. There is no point in your trying to optimize line and page breaks in the original manuscript. The manuscript will be reformatted to use the journal's preferred fonts and layout.

Proofs. Page proofs will be made available to authors (or to the designated corresponding author) at a Web site in PDF format. Failure to acknowledge the receipt of proofs or to return corrections within the requested deadline may cause publication to be postponed. 
Mathematics and Mechanics of Complex Systems vol. 4 no. 2

Constraint reaction and the Peach-Koehler force for dislocation networks

Riccardo Scala and Nicolas Van Goethem

Stability analysis of two coupled oscillators

Mickhail A. Guzev and Alexandr A. Dmitriev

Analysis of the electromagnetic reflection and transmission 153 through a stratified lossy medium of an elliptically

polarized plane wave

Fabio Mangini and Fabrizio Frezza

Dislocation-induced linear-elastic strain dynamics by a

Cahn-Hilliard-type equation

Nicolas Van Goethem

MEMOCS is a journal of the International Research Center for the Mathematics and Mechanics of Complex Systems at the Università dell' Aquila, Italy.

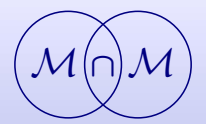

